JOURNAL OF THE

AMERICAN MATHEMATICAL SOCIETY

Volume 14, Number 4, Pages 941-1006

S 0894-0347(01)00373-3

Article electronically published on May 29, 2001

\title{
HILBERT SCHEMES, POLYGRAPHS AND THE MACDONALD POSITIVITY CONJECTURE
}

\author{
MARK HAIMAN
}

\section{INTRODUCTION}

The Hilbert scheme of points in the plane $H_{n}=\operatorname{Hilb}^{n}\left(\mathbb{C}^{2}\right)$ is an algebraic variety which parametrizes finite subschemes $S$ of length $n$ in $\mathbb{C}^{2}$. To each such subscheme $S$ corresponds an $n$-element multiset, or unordered $n$-tuple with possible repetitions, $\sigma(S)=\llbracket P_{1}, \ldots, P_{n} \rrbracket$ of points in $\mathbb{C}^{2}$, where the $P_{i}$ are the points of $S$, repeated with appropriate multiplicities. There is a variety $X_{n}$, finite over $H_{n}$, whose fiber over the point of $H_{n}$ corresponding to $S$ consists of all ordered $n$-tuples $\left(P_{1}, \ldots, P_{n}\right) \in\left(\mathbb{C}^{2}\right)^{n}$ whose underlying multiset is $\sigma(S)$. We call $X_{n}$ the isospectral Hilbert scheme.

By a theorem of Fogarty [14], the Hilbert scheme $H_{n}$ is irreducible and nonsingular. The geometry of $X_{n}$ is more complicated, but also very special. Our main geometric result, Theorem 3.1 is that $X_{n}$ is normal, Cohen-Macaulay and Gorenstein.

Earlier investigations by the author 24] unearthed indications of a far-reaching correspondence between the geometry and sheaf cohomology of $H_{n}$ and $X_{n}$ on the one hand, and the theory of Macdonald polynomials on the other. The Macdonald polynomials

$$
P_{\mu}(x ; q, t)
$$

are a basis of the algebra of symmetric functions in variables $x=x_{1}, x_{2}, \ldots$, with coefficients in the field $\mathbb{Q}(q, t)$ of rational functions in two parameters $q$ and $t$. They were introduced in 1988 by Macdonald [39] to unify the two well-known oneparameter bases of the algebra of symmetric functions, namely, the Hall-Littlewood polynomials and the Jack polynomials (for a thorough treatment see [40]). It promptly became clear that the discovery of Macdonald polynomials was fundamental and sure to have many ramifications. Developments in the years since have borne this out, notably, Cherednik's proof of the Macdonald constant-term identities 9 and other discoveries relating Macdonald polynomials to the representation theory of quantum groups [13] and affine Hecke algebras [32 33, 41, the CalogeroSutherland model in particle physics [35], and combinatorial conjectures on diagonal harmonics [3, 16, 22].

Received by the editors August 15, 2000 and, in revised form, January 29, 2001.

2000 Mathematics Subject Classification. Primary 14C05; Secondary 05E05, 14M05.

Key words and phrases. Macdonald polynomials, Hilbert schemes, Cohen-Macaulay, Gorenstein, sheaf cohomology.

This research was supported in part by N.S.F. Mathematical Sciences grants DMS-9701218 and DMS-0070772. 
The link between Macdonald polynomials and Hilbert schemes comes from work by Garsia and the author on the Macdonald positivity conjecture. The Schur function expansions of Macdonald polynomials lead to transition coefficients $K_{\lambda \mu}(q, t)$, known as Kostka-Macdonald coefficients. As defined, they are rational functions of $q$ and $t$, but conjecturally they are polynomials in $q$ and $t$ with nonnegative integer coefficients:

$$
K_{\lambda \mu}(q, t) \in \mathbb{N}[q, t] .
$$

The positivity conjecture has remained open since Macdonald formulated it at the time of his original discovery. For $q=0$ it reduces to the positivity theorem for $t$-Kostka coefficients, which has important algebraic, geometric and combinatorial interpretations 17, 10, 17, 27, 31, 34, 36, 37, 38, 45. Only recently have several authors independently shown that the Kostka-Macdonald coefficients are polynomials, $K_{\lambda \mu}(q, t) \in \mathbb{Z}[q, t]$, but these results do not establish the positivity 18, 19, 32, 33, 44 .

In [15, Garsia and the author conjectured an interpretation of the KostkaMacdonald coefficients $K_{\lambda \mu}(q, t)$ as graded character multiplicities for certain doubly graded $S_{n}$-modules $D_{\mu}$. The module $D_{\mu}$ is the space of polynomials in $2 n$ variables spanned by all derivatives of a certain simple determinant (see $\$ 2.2$ for the precise definition). The conjectured interpretation implies the Macdonald positivity conjecture. It also implies, in consequence of known properties of the $K_{\lambda \mu}(q, t)$, that for each partition $\mu$ of $n$, the dimension of $D_{\mu}$ is equal to $n$ !. This seemingly elementary assertion has come to be known as the $n$ ! conjecture.

It develops that these conjectures are closely tied to the geometry of the isospectral Hilbert scheme. Specifically, in [24] we were able to show that the CohenMacaulay property of $X_{n}$ is equivalent to the $n$ ! conjecture. We further showed that the Cohen-Macaulay property of $X_{n}$ implies the stronger conjecture interpreting $K_{\lambda \mu}(q, t)$ as a graded character multiplicity for $D_{\mu}$. Thus the geometric results in the present article complete the proof of the Macdonald positivity conjecture.

Another consequence of our results, equivalent in fact to our main theorem, is that the Hilbert scheme $H_{n}$ is equal to the $G$-Hilbert scheme $V / / G$ of Ito and Nakamura [28, for the case $V=\left(\mathbb{C}^{2}\right)^{n}, G=S_{n}$. The $G$-Hilbert scheme is of interest in connection with the generalized McKay correspondence, which says that if $V$ is a complex vector space, $G$ is a finite subgroup of $\mathrm{SL}(V)$ and $Y \rightarrow V / G$ is a so-called crepant resolution of singularities, then the sum of the Betti numbers of $Y$ equals the number of conjugacy classes of $G$. In many interesting cases [6, 42], the $G$-Hilbert scheme turns out to be a crepant resolution and an instance of the McKay correspondence. By our main theorem, this holds for $G=S_{n}, V=\left(\mathbb{C}^{2}\right)^{n}$.

We wish to say a little at this point about how the discoveries presented here came about. It has long been known [27, 45, that the $t$-Kostka coefficients $K_{\lambda \mu}(t)=$ $K_{\lambda \mu}(0, t)$ are graded character multiplicities for the cohomology rings of Springer fibers. Garsia and Procesi [17] found a new proof of this result, deriving it directly from an elementary description of the rings in question. In doing so, they hoped to reformulate the result for $K_{\lambda \mu}(t)$ in a way that might generalize to the twoparameter case. Shortly after that, Garsia and the author began their collaboration and soon found the desired generalization, in the form of the $n$ ! conjecture. Based on Garsia and Procesi's experience, we initially expected that the $n$ ! conjecture itself would be easy to prove and that the difficulties would lie in the identification 
of $K_{\lambda \mu}(q, t)$ as the graded character multiplicity. To our surprise, however, the $n$ ! conjecture stubbornly resisted elementary attack.

In the spring of 1992, we discussed our efforts on the $n$ ! conjecture with Procesi, along with another related conjecture we had stumbled upon in the course of our work. The modules involved in the $n$ ! conjecture are quotients of the ring $R_{n}$ of coinvariants for the action of $S_{n}$ on the polynomial ring in $2 n$ variables. This ring $R_{n}$ is isomorphic to the space of diagonal harmonics. Computations suggested that its dimension should be $(n+1)^{n-1}$ and that its graded character should be related to certain well-known combinatorial enumerations (this conjecture is discussed briefly in $\$ 5.3$ and at length in [16, 22]). Procesi suggested that the Hilbert scheme $H_{n}$ and what we now call the isospectral Hilbert scheme $X_{n}$ should be relevant to the determination of the dimension and character of $R_{n}$. Specifically, he observed that there is a natural map from $R_{n}$ to the ring of global functions on the schemetheoretic fiber in $X_{n}$ over the origin in $S^{n} \mathbb{C}^{2}$. With luck, this map might be an isomorphism, and - as we are now able to confirm - $X_{n}$ might be flat over $H_{n}$, so that its structure sheaf would push down to a vector bundle on $H_{n}$. Then $R_{n}$ would coincide with the space of global sections of this vector bundle over the zero-fiber in $H_{n}$, and it might be possible to compute its character using the Atiyah-Bott Lefschetz formula.

The connection between $X_{n}$ and the $n$ ! conjecture became clear when the author sought to carry out the computation Procesi had suggested, assuming the validity of some needed but unproven geometric hypotheses. More precisely, it became clear that the spaces in the $n$ ! conjecture should be the fibers of Procesi's vector bundle at distinguished torus-fixed points in $H_{n}$, a fact which we prove in $\$ 3.7$ These considerations ultimately led to a conjectured formula for the character of $R_{n}$ in terms of Macdonald polynomials. This formula turned out to be correct up to the limit of practical computation $(n \leq 7)$. Furthermore, Garsia and the author were able to show in [16] that the series of combinatorial conjectures in [22] would all follow from the conjectured master formula. Thus we had strong indications that Procesi's proposed picture was indeed valid, and that a geometric study of $X_{n}$ should ultimately lead to a proof of the $n$ ! and Macdonald positivity conjectures, as is borne out here. By now the reader should expect the geometric study of $X_{n}$ also to yield a proof of the character formula for diagonal harmonics and the $(n+1)^{n-1}$ conjecture. This subject will be taken up in a separate article.

The remainder of the paper is organized as follows. In $₫ 2$ we give the relevant definitions concerning Macdonald polynomials and state the positivity, $n$ ! and graded character conjectures. Hilbert scheme definitions and the statement and proof of the main theorem are in 93 , along with the equivalence of the main theorem to the $n$ ! conjecture. In $\$ 3.9$ we review the proof from [24 that the main theorem implies the conjecture of Garsia and the author on the character of the space $D_{\mu}$, and hence implies the Macdonald positivity conjecture.

The proof of the main theorem uses a technical result, Theorem 4.1, that the coordinate ring of a certain type of subspace arrangement we call a polygraph is a free module over the polynomial ring generated by some of the coordinates. Section 4 4 contains the definition and study of polygraphs, culminating in the proof of Theorem 4.1. At the end, in $\$ 5$, we discuss other implications of our results, including the connection with $G$-Hilbert schemes, along with related conjectures and open problems. 


\section{The $n$ ! AND MACDONALD POSITIVITy CONJECTURES}

2.1. Macdonald polynomials. We work with the transformed integral forms $\tilde{H}_{\mu}(x ; q, t)$ of the Macdonald polynomials, indexed by integer partitions $\mu$, and homogeneous of degree $n=|\mu|$. These are defined as in [24], eq. (2.18), to be

$$
\tilde{H}_{\mu}(x ; q, t)=t^{n(\mu)} J_{\mu}\left[X /\left(1-t^{-1}\right) ; q, t^{-1}\right],
$$

where $J_{\mu}$ denotes Macdonald's integral form as in [40], VI, eq. (8.3), and $n(\mu)$ is the partition statistic

$$
n(\mu)=\sum_{i}(i-1) \mu_{i}
$$

(not to be confused with $n=|\mu|$ ).

The square brackets in (3) stand for plethystic substitution. We pause briefly to review the definition of this operation (see [24 for a fuller discussion). Let $\mathbb{F}[[x]]$ be the algebra of formal series over the coefficient field $\mathbb{F}=\mathbb{Q}(q, t)$, in variables $x=x_{1}, x_{2}, \ldots$. For any $A \in \mathbb{F}[[x]]$, we denote by $p_{k}[A]$ the result of replacing each indeterminate in $A$ by its $k$-th power. This includes the indeterminates $q$ and $t$ as well as the variables $x_{i}$. The algebra of symmetric functions $\Lambda_{\mathbb{F}}$ is freely generated as an $\mathbb{F}$-algebra by the power-sums

$$
p_{k}(x)=x_{1}^{k}+x_{2}^{k}+\cdots .
$$

Hence there is a unique $\mathbb{F}$-algebra homomorphism

$$
\mathrm{ev}_{A}: \Lambda_{\mathbb{F}} \rightarrow \mathbb{F}[[x]] \quad \text { defined by } p_{k}(x) \mapsto p_{k}[A] .
$$

In general we write $f[A]$ for $\operatorname{ev}_{A}(f)$, for any $f \in \Lambda_{\mathbb{F}}$. With this notation goes the convention that $X$ stands for the sum $X=x_{1}+x_{2}+\cdots$ of the variables, so we have $p_{k}[X]=p_{k}(x)$ and hence $f[X]=f(x)$ for all $f$. Note that a plethystic substitution like $f \mapsto f\left[X /\left(1-t^{-1}\right)\right]$, such as we have on the right-hand side in (3), yields again a symmetric function.

There is a simple direct characterization of the transformed Macdonald polynomials $\tilde{H}_{\mu}$.

Proposition 2.1.1 ([24], Proposition 2.6). The $\tilde{H}_{\mu}(x ; q, t)$ satisfy

(1) $\tilde{H}_{\mu}(x ; q, t) \in \mathbb{Q}(q, t)\left\{s_{\lambda}[X /(1-q)]: \lambda \geq \mu\right\}$,

(2) $\tilde{H}_{\mu}(x ; q, t) \in \mathbb{Q}(q, t)\left\{s_{\lambda}[X /(1-t)]: \lambda \geq \mu^{\prime}\right\}$, and

(3) $\tilde{H}_{\mu}[1 ; q, t]=1$,

where $s_{\lambda}(x)$ denotes a Schur function, $\mu^{\prime}$ is the partition conjugate to $\mu$, and the ordering is the dominance partial order on partitions of $n=|\mu|$. These conditions characterize $\tilde{H}_{\mu}(x ; q, t)$ uniquely.

We set $\tilde{K}_{\lambda \mu}(q, t)=t^{n(\mu)} K_{\lambda \mu}\left(q, t^{-1}\right)$, where $K_{\lambda \mu}(q, t)$ is the Kostka-Macdonald coefficient defined in [40, VI, eq. (8.11). This is then related to the transformed Macdonald polynomials by

$$
\tilde{H}_{\mu}(x ; q, t)=\sum_{\lambda} \tilde{K}_{\lambda \mu}(q, t) s_{\lambda}(x) .
$$

It is known that $K_{\lambda \mu}(q, t)$ has degree at most $n(\mu)$ in $t$, so the positivity conjecture (22) from the introduction can be equivalently formulated in terms of $\tilde{K}_{\lambda \mu}$.

Conjecture 2.1.2 (Macdonald positivity conjecture). We have $\tilde{K}_{\lambda \mu}(q, t) \in \mathbb{N}[q, t]$. 


\subsection{The $n$ ! and graded character conjectures. Let}

$$
\mathbb{C}[\mathbf{x}, \mathbf{y}]=\mathbb{C}\left[x_{1}, y_{1}, \ldots, x_{n}, y_{n}\right]
$$

be the polynomial ring in $2 n$ variables. To each $n$-element subset $D \subseteq \mathbb{N} \times \mathbb{N}$, we associate a polynomial $\Delta_{D} \in \mathbb{C}[\mathbf{x}, \mathbf{y}]$ as follows. Let $\left(p_{1}, q_{1}\right), \ldots,\left(p_{n}, q_{n}\right)$ be the elements of $D$ listed in some fixed order. Then we define

$$
\Delta_{D}=\operatorname{det}\left(x_{i}^{p_{j}} y_{i}^{q_{j}}\right)_{1 \leq i, j \leq n} .
$$

If $\mu$ is a partition of $n$, its diagram is the set

$$
D(\mu)=\left\{(i, j): j<\mu_{i+1}\right\} \subseteq \mathbb{N} \times \mathbb{N} .
$$

(Note that in our definition the rows and columns of the diagram $D(\mu)$ are indexed starting with zero.) In the case where $D=D(\mu)$ is the diagram of a partition, we abbreviate

$$
\Delta_{\mu}=\Delta_{D(\mu)}
$$

The polynomial $\Delta_{\mu}(\mathbf{x}, \mathbf{y})$ is a kind of bivariate analog of the Vandermonde determinant $\Delta(\mathbf{x})$, which occurs as the special case $\mu=\left(1^{n}\right)$.

Given a partition $\mu$ of $n$, we denote by

$$
D_{\mu}=\mathbb{C}[\partial \mathbf{x}, \partial \mathbf{y}] \Delta_{\mu}
$$

the space spanned by all the iterated partial derivatives of $\Delta_{\mu}$. In [15], Garsia and the author proposed the following conjecture, which we will prove as a consequence of Proposition 3.7 .3 and Theorem 3.1

Conjecture 2.2.1 ( $n$ ! conjecture). The dimension of $D_{\mu}$ is equal to $n$ !.

The $n$ ! conjecture arose as part of a stronger conjecture relating the KostkaMacdonald coefficients to the character of $D_{\mu}$ as a doubly graded $S_{n}$-module. The symmetric group $S_{n}$ acts by $\mathbb{C}$-algebra automorphisms of $\mathbb{C}[\mathbf{x}, \mathbf{y}]$ permuting the variables:

$$
w x_{i}=x_{w(i)}, w y_{i}=y_{w(i)} \text { for } \quad w \in S_{n} .
$$

The ring $\mathbb{C}[\mathbf{x}, \mathbf{y}]=\bigoplus_{r, s} \mathbb{C}[\mathbf{x}, \mathbf{y}]_{r, s}$ is doubly graded, by degree in the $\mathbf{x}$ and $\mathbf{y}$ variables respectively, and the $S_{n}$ action respects the grading. Clearly $\Delta_{\mu}$ is $S_{n^{-}}$ alternating, i.e., we have $w \Delta_{\mu}=\epsilon(w) \Delta_{\mu}$ for all $w \in S_{n}$, where $\epsilon$ is the sign character. Note that $\Delta_{\mu}$ is also doubly homogeneous, of $x$-degree $n(\mu)$ and $y$-degree $n\left(\mu^{\prime}\right)$. It follows that the space $D_{\mu}$ is $S_{n}$-invariant and has a double grading

$$
D_{\mu}=\bigoplus_{r, s}\left(D_{\mu}\right)_{r, s}
$$

by $S_{n}$-invariant subspaces $\left(D_{\mu}\right)_{r, s}=D_{\mu} \cap \mathbb{C}[\mathbf{x}, \mathbf{y}]_{r, s}$.

We write $\operatorname{ch} V$ for the character of an $S_{n}$-module $V$, and denote the irreducible $S_{n}$ characters by $\chi^{\lambda}$, with the usual indexing by partitions $\lambda$ of $n$. The following conjecture implies the Macdonald positivity conjecture.

Conjecture 2.2.2 ([15]). We have

$$
\tilde{K}_{\lambda \mu}(q, t)=\sum_{r, s} t^{r} q^{s}\left\langle\chi^{\lambda}, \operatorname{ch}\left(D_{\mu}\right)_{r, s}\right\rangle
$$


Macdonald had shown that $K_{\lambda \mu}(1,1)$ is equal to $\chi^{\lambda}(1)$, the degree of the irreducible $S_{n}$ character $\chi^{\lambda}$, or the number of standard Young tableaux of shape $\lambda$. Conjecture 2.2.2 therefore implies that $D_{\mu}$ affords the regular representation of $S_{n}$. In particular, it implies the $n$ ! conjecture.

In 24 the author showed that Conjecture 2.2.2 would follow from the CohenMacaulay property of $X_{n}$. We summarize the argument proving Conjecture 2.2.2 in $\$ 3.9$, after the relevant geometric results have been established.

\section{The isospectral Hilbert scheme}

3.1. Preliminaries. In this section we define the isospectral Hilbert scheme $X_{n}$, and deduce our main theorem, Theorem 3.1 (\$3.8). We also define the Hilbert scheme $H_{n}$ and the nested Hilbert scheme $H_{n-1, n}$, and develop some basic properties of these various schemes in preparation for the proof of the main theorem.

The main technical device used in the proof of Theorem 3.1 is a theorem on certain subspace arrangements called polygraphs, Theorem 4.1. The proof of the latter theorem is lengthy and logically distinct from the geometric reasoning leading from there to Theorem 3.1. For these reasons we have deferred Theorem 4.1 and its proof to the separate $\S_{4}$

Throughout this section we work in the category of schemes of finite type over the field of complex numbers, $\mathbb{C}$. All the specific schemes we consider are quasiprojective over $\mathbb{C}$. We use classical geometric language, describing open and closed subsets of schemes, and morphisms between reduced schemes, in terms of closed points. A variety is a reduced and irreducible scheme.

Every locally free coherent sheaf $B$ of rank $n$ on a scheme $X$ of finite type over $\mathbb{C}$ is isomorphic to the sheaf of sections of an algebraic vector bundle of rank $n$ over $X$. For notational purposes, we identify the vector bundle with the sheaf $B$ and write $B(x)$ for the fiber of $B$ at a closed point $x \in X$. In sheaf-theoretic terms, the fiber is given by $B(x)=B \otimes_{\mathcal{O}_{X}}\left(\mathcal{O}_{X, x} / x\right)$.

A scheme $X$ is Cohen-Macaulay or Gorenstein if its local ring $\mathcal{O}_{X, x}$ at every point is a Cohen-Macaulay or Gorenstein local ring, respectively. For either condition it suffices that it holds at closed points $x$. At the end of the section, in 3.10 we provide a brief summary of the facts we need from duality theory and the theory of Cohen-Macaulay and Gorenstein schemes.

3.2. The schemes $H_{n}$ and $X_{n}$. Let $R=\mathbb{C}[x, y]$ be the coordinate ring of the affine plane $\mathbb{C}^{2}$. By definition, closed subschemes $S \subseteq \mathbb{C}^{2}$ are in one-to-one correspondence with ideals $I \subseteq R$. The subscheme $S=V(I)$ is finite if and only if $R / I$ has Krull dimension zero, or finite dimension as a vector space over $\mathbb{C}$. In this case, the length of $S$ is defined to be $\operatorname{dim}_{\mathbb{C}} R / I$.

The Hilbert scheme $H_{n}=\operatorname{Hilb}^{n}\left(\mathbb{C}^{2}\right)$ parametrizes finite closed subschemes $S \subseteq \mathbb{C}^{2}$ of length $n$. The scheme structure of $H_{n}$ and the precise sense in which it parametrizes the subschemes $S$ are defined by a universal property, which characterizes $H_{n}$ up to unique isomorphism. The universal property is actually a property of $H_{n}$ together with a closed subscheme $F \subseteq H_{n} \times \mathbb{C}^{2}$, called the universal family.

Proposition 3.2.1. There exist schemes $H_{n}=\operatorname{Hilb}^{n}\left(\mathbb{C}^{2}\right)$ and $F \subseteq H_{n} \times \mathbb{C}^{2}$ enjoying the following properties, which characterize them up to unique isomorphism:

(1) $F$ is flat and finite of degree $n$ over $H_{n}$, and 
(2) if $Y \subseteq T \times \mathbb{C}^{2}$ is a closed subscheme, flat and finite of degree $n$ over a scheme $T$, then there is a unique morphism $\phi: T \rightarrow H_{n}$ giving a commutative fiber product diagram

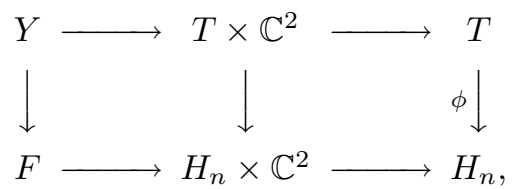

that is, the flat family $Y$ over $T$ is the pullback through $\phi$ of the universal family $F$.

Proof. The Hilbert scheme $\hat{H}=\operatorname{Hilb}^{n}\left(\mathbb{P}^{2}\right)$ of points in the projective plane exists as a special case of Grothendieck's construction in [21, with a universal family $\hat{F}$ having the analogous universal property. We identify $\mathbb{C}^{2}$ as usual with an open subset of $\mathbb{P}^{2}$, the complement of the projective line $Z$ "at infinity".

The projection of $\hat{F} \cap(\hat{H} \times Z)$ onto $\hat{H}$ is a closed subset of $\hat{H}$. Its complement $H_{n} \subseteq \hat{H}$ is clearly the largest subset such that the restriction $F$ of $\hat{F}$ to $H_{n}$ is contained in $H_{n} \times \mathbb{C}^{2}$. The required universal property of $H_{n}$ and $F$ now follows immediately from that of $\hat{H}$ and $\hat{F}$.

To see how $H_{n}$ parametrizes finite closed subschemes $S \subseteq \mathbb{C}^{2}$ of length $n$, note that the latter are exactly the families $Y$ in Proposition 3.2 .1 for $T=$ Spec $\mathbb{C}$. By the universal property they correspond one-to-one with the closed points of $H_{n}$, in such a way that the fiber of the universal family $F$ over the point corresponding to $S$ is $S$ itself. For notational purposes we will identify the closed points of $H_{n}$ with ideals $I \subseteq R$ satisfying $\operatorname{dim}_{\mathbb{C}} R / I=n$, rather than with the corresponding subschemes $S=V(I)$.

We have the following fundamental theorem of Fogarty [14].

Proposition 3.2.2. The Hilbert scheme $H_{n}$ is a nonsingular, irreducible variety over $\mathbb{C}$ of dimension $2 n$.

The generic examples of finite closed subschemes $S \subseteq \mathbb{C}^{2}$ of length $n$ are the reduced subschemes consisting of $n$ distinct points. They form an open subset of $H_{n}$, and the irreducibility aspect of Fogarty's theorem means that this open set is dense.

The most special closed subschemes in a certain sense are those defined by monomial ideals. If $I \subseteq R$ is a monomial ideal, then the standard monomials $x^{p} y^{q} \notin I$ form a basis of $R / I$. If $\operatorname{dim}_{\mathbb{C}} R / I=n$, the exponents $(p, q)$ of the standard monomials form the diagram $D(\mu)$ of a partition $\mu$ of $n$, and conversely. We use the partition $\mu$ to index the corresponding monomial ideal, denoting it by $I_{\mu}$. Note that $\sqrt{ } I_{\mu}=(x, y)$ for all $\mu$, so the subscheme $V\left(I_{\mu}\right)$ is concentrated at the origin $(0,0) \in \mathbb{C}^{2}$, and owes its length entirely to its nonreduced scheme structure.

The algebraic torus

$$
\mathbb{T}^{2}=\left(\mathbb{C}^{*}\right)^{2}
$$

acts on $\mathbb{C}^{2}$ as the group of invertible diagonal $2 \times 2$ matrices. The monomial ideals $I_{\mu}$ are the torus invariant ideals, and thus they are the fixed points of the induced action of $\mathbb{T}^{2}$ on the Hilbert scheme. Every ideal $I \in H_{n}$ has a monomial ideal in the closure of its $\mathbb{T}^{2}$-orbit ([23], Lemma 2.3). 
We write $x_{i}, y_{i}$ for the coordinates on the $i$-th factor in the Cartesian product $\left(\mathbb{C}^{2}\right)^{n}$, so we have $\left(\mathbb{C}^{2}\right)^{n}=\operatorname{Spec} \mathbb{C}[\mathbf{x}, \mathbf{y}]$, where $\mathbb{C}[\mathbf{x}, \mathbf{y}]=\mathbb{C}\left[x_{1}, y_{1}, \ldots, x_{n}, y_{n}\right]$. The symmetric group $S_{n}$ acts on $\left(\mathbb{C}^{2}\right)^{n}$ by permuting the factors. In coordinates, this corresponds to the action of $S_{n}$ on $\mathbb{C}[\mathbf{x}, \mathbf{y}]$ given in (13). We can identify Spec $\mathbb{C}[\mathbf{x}, \mathbf{y}]^{S_{n}}$ with the variety

$$
S^{n} \mathbb{C}^{2}=\left(\mathbb{C}^{2}\right)^{n} / S_{n}
$$

of unordered $n$-tuples, or $n$-element multisets, of points in $\mathbb{C}^{2}$.

Proposition 3.2.3 ([23], Proposition 2.2). For $I \in H_{n}$, let $\sigma(I)$ be the multiset of points of $V(I)$, counting each point $P$ with multiplicity equal to the length of the local ring $(R / I)_{P}$. Then the map $\sigma: H_{n} \rightarrow S^{n} \mathbb{C}^{2}$ is a projective morphism (called the Chow morphism).

Definition 3.2.4. The isospectral Hilbert scheme $X_{n}$ is the reduced fiber product

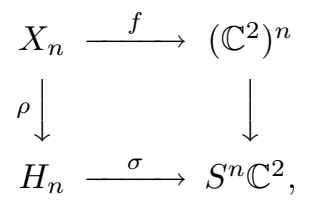

that is, the reduced closed subscheme of $H_{n} \times\left(\mathbb{C}^{2}\right)^{n}$ whose closed points are the tuples $\left(I, P_{1}, \ldots, P_{n}\right)$ satisfying $\sigma(I)=\llbracket P_{1}, \ldots, P_{n} \rrbracket$.

We will continue to refer to the morphisms $\rho, \sigma$ and $f$ in diagram (18) by those names in what follows.

For each $I \in H_{n}$, the operators $\bar{x}, \bar{y}$ of multiplication by $x, y$ are commuting endomorphisms of the $n$-dimensional vector space $R / I$. As such, they have a well-defined joint spectrum, a multiset of pairs of eigenvalues $\left(x_{1}, y_{1}\right), \ldots,\left(x_{n}, y_{n}\right)$ determined by the identity

$$
\operatorname{det}_{R / I}(1+\alpha \bar{x}+\beta \bar{y})=\prod_{i=1}^{n}\left(1+\alpha x_{i}+\beta y_{i}\right) .
$$

On the local ring $(R / I)_{P}$ at a point $P=\left(x_{0}, y_{0}\right)$, the operators $\bar{x}, \bar{y}$ have the sole joint eigenvalue $\left(x_{0}, y_{0}\right)$, with multiplicity equal to the length of $(R / I)_{P}$. Hence $\sigma(I)$ is equal as a multiset to the joint spectrum of $\bar{x}$ and $\bar{y}$. This is the motivation for the term isospectral.

The action of $S_{n}$ on $\left(\mathbb{C}^{2}\right)^{n}$ induces a compatible action of $S_{n}$ on $X_{n}$ by automorphisms of $X_{n}$ as a scheme over $H_{n}$. Explicitly, for $w \in S_{n}$ we have $w\left(I, P_{1}, \ldots, P_{n}\right)=$ $\left(I, P_{w^{-1}(1)}, \ldots, P_{w^{-1}(n)}\right)$.

We caution the reader that the scheme-theoretic fiber product in (18) is not reduced, even for $n=2$. For every invariant polynomial $g \in \mathbb{C}[\mathbf{x}, \mathbf{y}]^{S_{n}}$, the global regular function

$$
g\left(x_{1}, y_{1}, \ldots, x_{n}, y_{n}\right)-\sigma^{*} g
$$

on $H_{n} \times\left(\mathbb{C}^{2}\right)^{n}$ vanishes on $X_{n}$. By definition these equations generate the ideal sheaf of the scheme-theoretic fiber product. They cut out $X_{n}$ set-theoretically, but not as a reduced subscheme. The full ideal sheaf defining $X_{n}$ as a reduced scheme must necessarily have a complicated local description, since it is a consequence of Theorem 3.1 and Proposition 3.7.3, below, that generators for all the ideals $J_{\mu}$ in \$3.7] eq. (36), are implicit in the local ideals of $X_{n}$ at the distinguished points $Q_{\mu}$ lying over the torus-fixed points $I_{\mu} \in H_{n}$. 
3.3. Elementary properties of $X_{n}$. We now develop some elementary facts about the isospectral Hilbert scheme $X_{n}$. The first of these is its product structure, which allows us to reduce local questions on $X_{n}$ to questions about $X_{k}$ for $k<n$, in a neighborhood of any point whose corresponding multiset $\llbracket P_{1}, \ldots, P_{n} \rrbracket$ is not of the form $\llbracket n \cdot P \rrbracket$.

Lemma 3.3.1. Let $k$ and $l$ be positive integers with $k+l=n$. Suppose $U \subseteq\left(\mathbb{C}^{2}\right)^{n}$ is an open set consisting of points $\left(P_{1}, \ldots, P_{k}, Q_{1}, \ldots, Q_{l}\right)$ where no $P_{i}$ coincides with any $Q_{j}$. Then, identifying $\left(\mathbb{C}^{2}\right)^{n}$ with $\left(\mathbb{C}^{2}\right)^{k} \times\left(\mathbb{C}^{2}\right)^{l}$, the preimage $f^{-1}(U)$ of $U$ in $X_{n}$ is isomorphic as a scheme over $\left(\mathbb{C}^{2}\right)^{n}$ to the preimage $\left(f_{k} \times f_{l}\right)^{-1}(U)$ of $U$ in $X_{k} \times X_{l}$.

Proof. Let $Y=\left(\rho \times 1_{\mathbb{C}^{2}}\right)^{-1}(F) \subseteq X_{n} \times \mathbb{C}^{2}$ be the universal family over $X_{n}$. The fiber $V(I)$ of $Y$ over a point $\left(I, P_{1}, \ldots, P_{k}, Q_{1}, \ldots, Q_{l}\right) \in f^{-1}(U)$ is the disjoint union of closed subschemes $V\left(I_{k}\right)$ and $V\left(I_{l}\right)$ in $\mathbb{C}^{2}$ of lengths $k$ and $l$, respectively, with $\sigma\left(I_{k}\right)=\llbracket P_{1}, \ldots, P_{k} \rrbracket$ and $\sigma\left(I_{l}\right)=\llbracket Q_{1}, \ldots, Q_{l} \rrbracket$. Hence over $f^{-1}(U), Y$ is the disjoint union of flat families $Y_{k}, Y_{l}$ of degrees $k$ and $l$. By the universal property, we get induced morphisms $\phi_{k}: f^{-1}(U) \rightarrow H_{k}, \phi_{l}: f^{-1}(U) \rightarrow H_{l}$ and $\phi_{k} \times$ $\phi_{l}: f^{-1}(U) \rightarrow H_{k} \times H_{l}$. The equations $\sigma\left(I_{k}\right)=\llbracket P_{1}, \ldots, P_{k} \rrbracket, \sigma\left(I_{l}\right)=\llbracket Q_{1}, \ldots, Q_{l} \rrbracket$ imply that $\phi_{k} \times \phi_{l}$ factors through a morphism $\alpha: f^{-1}(U) \rightarrow X_{k} \times X_{l}$ of schemes over $\left(\mathbb{C}^{2}\right)^{n}$.

Conversely, on $\left(f_{k} \times f_{l}\right)^{-1}(U) \subseteq X_{k} \times X_{l}$, the pullbacks of the universal families from $X_{k}$ and $X_{l}$ are disjoint and their union is a flat family of degree $n$. By the universal property there is an induced morphism $\psi:\left(f_{k} \times f_{l}\right)^{-1}(U) \rightarrow H_{n}$, which factors through a morphism $\beta:\left(f_{k} \times f_{l}\right)^{-1}(U) \rightarrow X_{n}$ of schemes over $\left(\mathbb{C}^{2}\right)^{n}$.

By construction, the universal families on $f^{-1}(U)$ and $\left(f_{k} \times f_{l}\right)^{-1}(U)$ pull back to themselves via $\beta \circ \alpha$ and $\alpha \circ \beta$, respectively. This implies that $\beta \circ \alpha$ is a morphism of schemes over $H_{n}$ and $\alpha \circ \beta$ is a morphism of schemes over $H_{k} \times H_{l}$. Since they are also morphisms of schemes over $\left(\mathbb{C}^{2}\right)^{n}$, we have $\beta \circ \alpha=1_{f^{-1}(U)}$ and $\alpha \circ \beta=1_{\left(f_{k} \times f_{l}\right)^{-1}(U)}$. Hence $\alpha$ and $\beta$ induce mutually inverse isomorphisms $f^{-1}(U) \cong\left(f_{k} \times f_{l}\right)^{-1}(U)$.

Proposition 3.3.2. The isospectral Hilbert scheme $X_{n}$ is irreducible, of dimension $2 n$.

Proof. Let $U$ be the preimage in $X_{n}$ of the open set $W \subseteq\left(\mathbb{C}^{2}\right)^{n}$ consisting of points $\left(P_{1}, \ldots, P_{n}\right)$ where the $P_{i}$ are all distinct. It follows from Lemma 3.3 .1 that $f$ restricts to an isomorphism $f: U \rightarrow W$, so $U$ is irreducible. We are to show that $U$ is dense in $X_{n}$.

Let $Q$ be a closed point of $X_{n}$, which we want to show belongs to the closure $\bar{U}$ of $U$. If $f(Q)=\left(P_{1}, \ldots, P_{n}\right)$ with the $P_{i}$ not all equal, then by Lemma 3.3.1 there is a neighborhood of $Q$ in $X_{n}$ isomorphic to an open set in $X_{k} \times X_{l}$ for some $k, l<n$. The result then follows by induction, since we may assume $X_{k}$ and $X_{l}$ irreducible. If all the $P_{i}$ are equal, then $Q$ is the unique point of $X_{n}$ lying over $I=\rho(Q) \in H_{n}$. Since $\rho$ is finite, $\rho(\bar{U}) \subseteq H_{n}$ is closed. But $\rho(U)$ is dense in $H_{n}$, so $\rho(\bar{U})=H_{n}$. Therefore $\bar{U}$ contains a point lying over $I$, which must be $Q$.

Proposition 3.3.3. The closed subset $V\left(y_{1}, \ldots, y_{n}\right)$ in $X_{n}$ has dimension $n$.

Proof. It follows from the cell decomposition of Ellingsrud and Strømme [11] that the closed locus $Z$ in $H_{n}$ consisting of points $I$ with $V(I)$ supported on the $x$-axis $V(y)$ in $\mathbb{C}^{2}$ is the union of locally closed affine cells of dimension $n$. The subset $V(\mathbf{y}) \subseteq X_{n}$ is equal to $\rho^{-1}(Z)$ and $\rho$ is finite. 
The product structure of $X_{n}$ is inherited in a certain sense by $H_{n}$, but its description in terms of $X_{n}$ is more transparent. As a consequence, passage to $X_{n}$ is sometimes handy for proving results purely about $H_{n}$. The following lemma is an example of this. We remark that one can show by a more careful analysis that locus $G_{r}$ in the lemma is in fact irreducible.

Lemma 3.3.4. Let $G_{r}$ be the closed subset of $H_{n}$ consisting of ideals $I$ for which $\sigma(I)$ contains some point with multiplicity at least $r$. Then $G_{r}$ has codimension $r-1$, and has a unique irreducible component of maximal dimension.

Proof. By symmetry among the points $P_{i}$ of $\sigma(I)$ we see that $G_{r}=\rho\left(V_{r}\right)$, where $V_{r}$ is the locus in $X_{n}$ defined by the equations $P_{1}=\cdots=P_{r}$. It follows from Lemma 3.3.1 that $V_{r} \backslash \rho^{-1}\left(G_{r+1}\right)$ is isomorphic to an open set in $W_{r} \times X_{n-r}$, where $W_{r}$ is the closed subset $P_{1}=\cdots=P_{r}$ in $X_{r}$. As a reduced subscheme of $X_{r}$, the latter is isomorphic to $\mathbb{C}^{2} \times Z_{r}$, where $Z_{r}=\sigma^{-1}(\underline{0})$ is the zero fiber in $H_{r}$, the factor $\mathbb{C}^{2}$ accounting for the choice of $P=P_{1}=\cdots=P_{r}$.

By a theorem of Briançon [5], $Z_{r}$ is irreducible of dimension $r-1$, so $V_{r} \backslash$ $\rho^{-1}\left(G_{r+1}\right)$ is irreducible of dimension $2(n-r)+r+1=2 n-(r-1)$. Since $G_{r} \backslash G_{r+1}=\rho\left(V_{r} \backslash \rho^{-1}\left(G_{r+1}\right)\right)$ and $\rho$ is finite, the result follows by descending induction on $r$, starting with $G_{n+1}=\emptyset$.

3.4. Blowup construction of $H_{n}$ and $X_{n}$. Let $A=\mathbb{C}[\mathbf{x}, \mathbf{y}]^{\epsilon}$ be the space of $S_{n^{-}}$ alternating elements, that is, polynomials $g$ such that $w g=\epsilon(w) g$ for all $w \in S_{n}$, where $\epsilon$ is the sign character. To describe $A$ more precisely, we note that $A$ is the image of the alternation operator

$$
\Theta^{\epsilon} g=\sum_{w \in S_{n}} \epsilon(w) w g
$$

If $D=\left\{\left(p_{1}, q_{1}\right), \ldots,\left(p_{n}, q_{n}\right)\right\}$ is an $n$-element subset of $\mathbb{N} \times \mathbb{N}$, then the determinant $\Delta_{D}$ defined in (9) can also be written

$$
\Delta_{D}=\Theta^{\epsilon}\left(\mathbf{x}^{p} \mathbf{y}^{q}\right),
$$

where $\mathbf{x}^{p} \mathbf{y}^{q}=x_{1}^{p_{1}} y_{1}^{q_{1}} \cdots x_{n}^{p_{n}} y_{n}^{q_{n}}$. For a monomial $\mathbf{x}^{p} \mathbf{y}^{q}$ whose exponent pairs $\left(p_{i}, q_{i}\right)$ are not all distinct, we have $\Theta^{\epsilon}\left(\mathbf{x}^{p} \mathbf{y}^{q}\right)=0$. From this it is easy to see that the set of all elements $\Delta_{D}$ is a basis of $A$. Another way to see this is to identify $A$ with the $n$-th exterior power $\wedge^{n} \mathbb{C}[x, y]$ of the polynomial ring in two variables $x, y$. Then the basis elements $\Delta_{D}$ are identified with the wedge products of monomials in $\mathbb{C}[x, y]$.

For $d>0$, let $A^{d}$ be the space spanned by all products of $d$ elements of $A$. We set $A^{0}=\mathbb{C}[\mathbf{x}, \mathbf{y}]^{S_{n}}$. Note that $A$ and hence every $A^{d}$ is a $\mathbb{C}[\mathbf{x}, \mathbf{y}]^{S_{n}}$-submodule of $\mathbb{C}[\mathbf{x}, \mathbf{y}]$, so we have $A^{i} A^{j}=A^{i+j}$ for all $i, j$, including $i=0$ or $j=0$.

Proposition 3.4.1 ([23], Proposition 2.6). The Hilbert scheme $H_{n}$ is isomorphic as a scheme projective over $S^{n} \mathbb{C}^{2}$ to $\operatorname{Proj} T$, where $T$ is the graded $\mathbb{C}[\mathbf{x}, \mathbf{y}]^{S_{n}}$-algebra $T=\bigoplus_{d \geq 0} A^{d}$.

Proposition 3.4.2. The isospectral Hilbert scheme $X_{n}$ is isomorphic as a scheme over $\left(\mathbb{C}^{2}\right)^{n}$ to the blowup of $\left(\mathbb{C}^{2}\right)^{n}$ at the ideal $J=\mathbb{C}[\mathbf{x}, \mathbf{y}] A$ generated by the alternating polynomials.

Proof. Set $S=\mathbb{C}[\mathbf{x}, \mathbf{y}]$. By definition the blowup of $\left(\mathbb{C}^{2}\right)^{n}$ at $J$ is $Z=\operatorname{Proj} S[t J]$, where $S[t J] \cong \bigoplus_{d \geq 0} J^{d}$ is the Rees algebra. The ring $T$ is a homogeneous subring of $S[t J]$ in an obvious way, and since $A^{d}$ generates $J^{d}$ as a $\mathbb{C}[\mathbf{x}, \mathbf{y}]$-module, we 
have $S \cdot T=S[t J]$, that is, $S[t J] \cong\left(\mathbb{C}[\mathbf{x}, \mathbf{y}] \otimes_{A^{0}} T\right) / I$ for some homogeneous ideal $I$. In geometric terms, using Proposition 3.4 .1 and the fact that $S^{n} \mathbb{C}^{2}=$ Spec $A^{0}$, this says that $Z$ is a closed subscheme of the scheme-theoretic fiber product $H_{n} \times S_{S^{n} \mathbb{C}^{2}}\left(\mathbb{C}^{2}\right)^{n}$. Since $Z$ is reduced, it follows that $Z$ is a closed subscheme of $X_{n}$. By Proposition 3.3.2 $X_{n}$ is irreducible, and since both $Z$ and $X_{n}$ have dimension $2 n$, it follows that $Z=X_{n}$.

In the context of either $H_{n}$ or $X_{n}$ we will always write $\mathcal{O}(k)$ for the $k$-th tensor power of the ample line bundle $\mathcal{O}(1)$ induced by the representation of $H_{n}$ as $\operatorname{Proj} T$ or $X_{n}$ as $\operatorname{Proj} S[t J]$. It is immediate from the proof of Proposition [3.4.2 that $\mathcal{O}_{X_{n}}(k)=\rho^{*} \mathcal{O}_{H_{n}}(k)$.

In full analogy to the situation for the Plücker embedding of a Grassmann variety, there is an intrinsic description of $\mathcal{O}(1)$ as the highest exterior power of the tautological vector bundle whose fiber at a point $I \in H_{n}$ is $R / I$. Let

$$
\pi: F \rightarrow H_{n}
$$

be the projection of the universal family on the Hilbert scheme. Since $\pi$ is an affine morphism, we have $F=\operatorname{Spec} B$, where $B$ is the sheaf of $\mathcal{O}_{H_{n}}$-algebras

$$
B=\pi_{*} \mathcal{O}_{F} .
$$

The fact that $F$ is flat and finite of degree $n$ over $H_{n}$ means that $B$ is a locally free sheaf of $\mathcal{O}_{H_{n}}$-modules of rank $n$. Its associated vector bundle is the tautological bundle.

Proposition 3.4.3 ([23], Proposition 2.12). We have an isomorphism $\wedge^{n} B \cong$ $\mathcal{O}(1)$ of line bundles on $H_{n}$.

3.5. Nested Hilbert schemes. The proof of our main theorem will be by induction on $n$. For the inductive step we interpolate between $H_{n-1}$ and $H_{n}$ using the nested Hilbert scheme.

Definition 3.5.1. The nested Hilbert scheme $H_{n-1, n}$ is the reduced closed subscheme

$$
H_{n-1, n}=\left\{\left(I_{n-1}, I_{n}\right): I_{n} \subseteq I_{n-1}\right\} \subseteq H_{n-1} \times H_{n} .
$$

The analog of Fogarty's theorem (Proposition 3.2.2) for the nested Hilbert scheme is the following result of Tikhomirov, whose proof can be found in [8].

Proposition 3.5.2. The nested Hilbert scheme $H_{n-1, n}$ is nonsingular and irreducible, of dimension $2 n$.

As with $H_{n}$, the nested Hilbert scheme is an open set in a projective nested Hilbert scheme $\operatorname{Hilb}^{n-1, n}\left(\mathbb{P}^{2}\right)$. Clearly, $H_{n-1, n}$ is the preimage of $H_{n}$ under the projection $\operatorname{Hilb}^{n-1, n}\left(\mathbb{P}^{2}\right) \rightarrow \operatorname{Hilb}^{n}\left(\mathbb{P}^{2}\right)$. Hence the projection $H_{n-1, n} \rightarrow H_{n}$ is a projective morphism.

If $\left(I_{n-1}, I_{n}\right)$ is a point of $H_{n-1, n}$, then $\sigma\left(I_{n-1}\right)$ is an $n-1$ element sub-multiset of $\sigma\left(I_{n}\right)$. In symbols, if $\sigma\left(I_{n-1}\right)=\llbracket P_{1}, \ldots, P_{n-1} \rrbracket$, then $\sigma\left(I_{n}\right)=\llbracket P_{1}, \ldots, P_{n-1}, P_{n} \rrbracket$ for a distinguished last point $P_{n}$. The $S_{n-1}$-invariant polynomials in the coordinates $x_{1}, y_{1}, \ldots, x_{n-1}, y_{n-1}$ of the points $P_{1}, \ldots, P_{n-1}$ are global regular functions on $H_{n-1, n}$, pulled back via the projection on $H_{n-1}$. Similarly, the $S_{n}$-invariant polynomials in $x_{1}, y_{1}, \ldots, x_{n}, y_{n}$ are regular functions pulled back from $H_{n}$. It follows that the coordinates of the distinguished point $P_{n}$ are regular functions, since 
$x_{n}=\left(x_{1}+\cdots+x_{n}\right)-\left(x_{1}+\cdots+x_{n-1}\right)$, and similarly for $y_{n}$. Thus we have a morphism

$$
\sigma: H_{n-1, n} \rightarrow S^{n-1} \mathbb{C}^{2} \times \mathbb{C}^{2}=\left(\mathbb{C}^{2}\right)^{n} / S_{n-1},
$$

such that both the maps $H_{n-1, n} \rightarrow S^{n-1} \mathbb{C}^{2}$ and $H_{n-1, n} \rightarrow S^{n} \mathbb{C}^{2}$ induced by the Chow morphisms composed with the projections on $H_{n-1}$ and $H_{n}$ factor through $\sigma$.

The distinguished point $P_{n}$ belongs to $V\left(I_{n}\right)$, and given $I_{n}$, every point of $V\left(I_{n}\right)$ occurs as $P_{n}$ for some choice of $I_{n-1}$. Therefore the image of the morphism

$$
\alpha: H_{n-1, n} \rightarrow H_{n} \times \mathbb{C}^{2}
$$

sending $\left(I_{n-1}, I_{n}\right)$ to $\left(I_{n}, P_{n}\right)$ is precisely the universal family $F$ over $H_{n}$. For clarity, let us point out that by the definition of $F$, we have $F=\left\{(I, P) \in H_{n} \times \mathbb{C}^{2}: P \in\right.$ $V(I)\}$, at least set-theoretically. In fact, $F$ is reduced and hence coincides as a reduced closed subscheme with this subset of $H_{n} \times \mathbb{C}^{2}$. This is true because $F$ is flat over the variety $H_{n}$ and generically reduced (see also the proof of Proposition 3.7 .2 below).

The following proposition, in conjunction with Lemma 3.3.4, provides dimension estimates needed for the calculation of the canonical line bundle on $H_{n-1, n}$ in $\$ 3.6$ and the proof of the main theorem in $\$ 3.8$.

Proposition 3.5.3. Let $d$ be the dimension of the fiber of the morphism $\alpha$ in (27) over a point $(I, P) \in F$, and let $r$ be the multiplicity of $P$ in $\sigma(I)$. Then $d$ and $r$ satisfy the inequality

$$
r \geq\left(\begin{array}{c}
d+2 \\
2
\end{array}\right)
$$

Proof. Recall that the socle of an Artin local ring $A$ is the ideal consisting of elements annihilated by the maximal ideal $m$. If $A$ is an algebra over a field $k$, with $A / m \cong k$, then every linear subspace of the socle is an ideal, and conversely every ideal in $A$ of length 1 is a one-dimensional subspace of the socle. The possible ideals $I_{n-1}$ for the given $\left(I_{n}, P_{n}\right)=(I, P)$ are the length 1 ideals in the Artin local $\mathbb{C}$-algebra $(R / I)_{P}$, where $R=\mathbb{C}[x, y]$. The fiber of $\alpha$ is therefore the projective space $\mathbb{P}\left(\operatorname{soc}(R / I)_{P}\right)$, and we have $d+1=\operatorname{dim} \operatorname{soc}(R / I)_{P}$.

First consider the maximum possible dimension of any fiber of $\alpha$. Since both $H_{n-1, n}$ and $F$ are projective over $H_{n}$, the morphism $\alpha$ is projective and its fiber dimension is upper semicontinuous. Since every point of $H_{n}$ has a monomial ideal $I_{\mu}$ in the closure of its $\mathbb{T}^{2}$-orbit, and since $F$ is finite over $H_{n}$, every point of $F$ must have a pair $\left(I_{\mu}, 0\right) \in F$ in the closure of its orbit. The fiber dimension is therefore maximized at some such point. The socle of $R / I_{\mu}$ has dimension equal to the number of corners of the diagram of $\mu$. If this number is $s$, we clearly have $n \geq\left(\begin{array}{c}s+1 \\ 2\end{array}\right)$. This implies that for every Artin local $\mathbb{C}$-algebra $R / I$ generated by two elements, the socle dimension $s$ and the length $n$ of $R / I$ satisfy $n \geq\left(\begin{array}{c}s+1 \\ 2\end{array}\right)$.

Returning to the original problem, $(R / I)_{P}$ is an Artin local $\mathbb{C}$-algebra of length $r$ generated by two elements, with socle dimension $d+1$, so (28) follows.

We now introduce the nested version of the isospectral Hilbert scheme. It literally plays a pivotal role in the proof of the main theorem by induction on $n$ : we transfer the Gorenstein property from $X_{n-1}$ to the nested scheme $X_{n-1, n}$ by pulling back, and from there to $X_{n}$ by pushing forward. 
Definition 3.5.4. The nested isospectral Hilbert scheme $X_{n-1, n}$ is the reduced fiber product $H_{n-1, n} \times_{H_{n-1}} X_{n-1}$.

There is an alternative formulation of the definition, which is useful to keep in mind for the next two results. Namely, $X_{n-1, n}$ can be identified with the reduced fiber product in the diagram

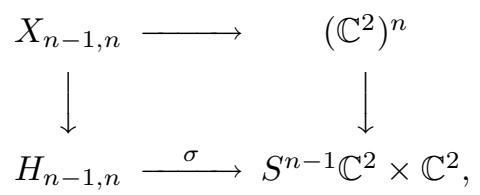

that is, the reduced closed subscheme of $H_{n-1, n} \times\left(\mathbb{C}^{2}\right)^{n}$ consisting of tuples $\left(I_{n-1}, I_{n}, P_{1}, \ldots, P_{n}\right)$ such that $\sigma\left(I_{n}\right)=\llbracket P_{1}, \ldots, P_{n} \rrbracket$ and $P_{n}$ is the distinguished point. To see that this agrees with the definition, note that a point of $H_{n-1, n} \times_{H_{n-1}}$ $X_{n-1}$ is given by the data $\left(I_{n-1}, I_{n}, P_{1}, \ldots, P_{n-1}\right)$, and that these data determine the distinguished point $P_{n}$. We obtain the alternative description by identifying $X_{n-1, n}$ with the graph in $X_{n-1, n} \times \mathbb{C}^{2}$ of the morphism $X_{n-1, n} \rightarrow \mathbb{C}^{2}$ sending $\left(I_{n-1}, I_{n}, P_{1}, \ldots, P_{n-1}\right)$ to $P_{n}$.

We have the following nested analogs of Lemma 3.3.1 and Proposition 3.3.3 The analog of Proposition 3.3.2 also holds, i.e., $X_{n-1, n}$ is irreducible. We do not prove this here, as it will follow automatically as part of our induction (see the observations following diagram (52) in $\$ 3.8$ ).

Lemma 3.5.5. Let $k+l=n$ and $U \subseteq\left(\mathbb{C}^{2}\right)^{n}$ be as in Lemma 3.3.1. Then the preimage of $U$ in $X_{n-1, n}$ is isomorphic as a scheme over $\left(\mathbb{C}^{2}\right)^{n}$ to the preimage of $U$ in $X_{k} \times X_{l-1, l}$.

Proof. Lemma 3.3 .1 gives us isomorphisms on the preimage of $U$ between $X_{n}$ and $X_{k} \times X_{l}$, and between $X_{n-1}$ and $X_{k} \times X_{l-1}$.

We can identify $X_{n-1, n}$ with the closed subset of $X_{n-1} \times X_{n}$ consisting of points where $P_{1}, \ldots, P_{n-1}$ are the same in both factors, and $I_{n-1}$ contains $I_{n}$. On the preimage of $U$, under the isomorphisms above, this corresponds to the closed subset of $\left(X_{k} \times X_{l-1}\right) \times\left(X_{k} \times X_{l}\right)$ where $P_{1}, \ldots, P_{k}, Q_{1}, \ldots, Q_{l-1}$ and $I_{k}$ are the same in both factors and $I_{l-1}$ contains $I_{l}$. The latter can be identified with $X_{k} \times X_{l-1, l}$.

Proposition 3.5.6. The closed subset $V\left(y_{1}, \ldots, y_{n}\right)$ in $X_{n-1, n}$ has dimension $n$.

Proof. We have the corresponding result for $X_{n}$ in Proposition 3.3.3 We can assume by induction that the result for the nested scheme holds for smaller values of $n$ (for the base case note that $X_{0,1} \cong X_{1} \cong \mathbb{C}^{2}$ ). Locally on a neighborhood of any point where $P_{1}, \ldots, P_{n}$ are not all equal, the result then follows from Lemma 3.5.5.

The locus where all the $P_{i}$ are equal is isomorphic to $\mathbb{C}^{1} \times Z$ where $Z=\sigma^{-1}(\underline{0}) \subseteq$ $H_{n-1, n}$ is the zero fiber in the nested Hilbert scheme, the factor $\mathbb{C}^{1}$ accounting for the choice of the common point $P=P_{1}=\cdots=P_{n}$ on the $x$-axis $V(y) \subseteq \mathbb{C}^{2}$. By a theorem of Cheah ([8], Theorem 3.3.3, part (5)) we have $\operatorname{dim} Z=n-1$.

3.6. Calculation of canonical line bundles. We will need to know the canonical sheaves $\omega$ on the smooth schemes $H_{n}$ and $H_{n-1, n}$. To compute them we make use of the fact that invertible sheaves on a normal variety are isomorphic if they have isomorphic restrictions to an open set whose complement has codimension at least two. 
Definition 3.6.1. Let $z=a x+b y$ be a linear form in the variables $x, y$. We denote by $U_{z}$ the open subset of $H_{n}$ consisting of ideals $I$ for which $z$ generates $R / I$ as an algebra over $\mathbb{C}$. We also denote by $U_{z}$ the preimage of $U_{z}$ under the projection $H_{n-1, n} \rightarrow H_{n}$.

Note that $z$ generates $R / I$ if and only if the set $\left\{1, z, \ldots, z^{n-1}\right\}$ is linearly independent, and thus a basis, in $R / I$. That given sections of a vector bundle determine linearly independent elements in its fiber at a point $I$ is an open condition on $I$, so $U_{z}$ is indeed an open subset of $H_{n}$. The ring $R / I$ can be generated by a single linear form $z$ if and only if the scheme $V(I)$ is a subscheme of a smooth curve in $\mathbb{C}^{2}$. For this reason the union of all the open sets $U_{z}$ is called the curvilinear locus.

Lemma 3.6.2. The complement of $U_{x} \cup U_{y}$ has codimension 2 , both in $H_{n}$ and in $H_{n-1, n}$.

Proof. Let $Z=H_{n} \backslash\left(U_{x} \cup U_{y}\right)$. Let $W$ be the generic locus, that is, the open set of ideals $I \in H_{n}$ for which $\sigma(I)=\left\{P_{1}, \ldots, P_{n}\right\}$ is a set of $n$ distinct points. The Chow morphism $\sigma$ induces an isomorphism of $W$ onto its image in $S^{n} \mathbb{C}^{2}$, and $Z \cap W$ is the locus where some two of the $P_{i}$ have the same $x$-coordinate, and another two have the same $y$-coordinate. This locus has codimension 2 . The complement of $W$ is the closed subset $G_{2}$ in Lemma 3.3.4, which has one irreducible component of dimension $2 n-1$. An open set in this component consists of those $I$ for which $\sigma(I)$ has one point $P_{i}$ of multiplicity 2 and the rest are distinct. This open set is not contained in $Z$, so $Z \cap G_{2}$ has codimension at least 2. This takes care of $H_{n}$.

If $I$ is curvilinear, then the socle of $(R / I)_{P}$ has length 1 for all $P \in V(I)$. Hence the morphism $\alpha: H_{n-1, n} \rightarrow F$ in (27) restricts to a bijection on the curvilinear locus. If the complement of $U_{x} \cup U_{y}$ in $H_{n-1, n}$ had a codimension 1 component, it would therefore have to be contained in the complement of the curvilinear locus, by the result for $H_{n}$.

By Proposition 3.5.3, $\alpha$ has fibers of dimension $d$ only over $G_{r}$ for $r \geq\left(\begin{array}{c}d+2 \\ 2\end{array}\right)$, and it follows from Lemma 3.3 .4 that the union of these fibers has codimension at least $\left(\begin{array}{c}d+2 \\ 2\end{array}\right)-1-d=\left(\begin{array}{c}d+1 \\ 2\end{array}\right)$. For $d>1$ this exceeds 2. The fibers of dimension 1 over $G_{3}$ occur only over noncurvilinear points. However, by Lemma 3.3.4, there is a unique codimension 2 component of $G_{3}$. This component contains all $I$ such that $\sigma(I)$ has one point of multiplicity 3 and the rest distinct, and such an $I$ can be curvilinear. Hence the noncurvilinear locus in $G_{3}$ has codimension at least 3 and its preimage in $H_{n-1, n}$ has codimension at least 2 .

The following proposition is well known; it holds for the Hilbert scheme of points on any smooth surface with trivial canonical sheaf. We give an elementary proof for $H_{n}$, since we need it as a starting point for the proof of the corresponding result for $H_{n-1, n}$.

Proposition 3.6.3. The canonical sheaf $\omega_{H_{n}}$ on the Hilbert scheme is trivial, i.e., $\omega_{H_{n}} \cong \mathcal{O}_{H_{n}}$.

Proof. The $2 n$-form $d \mathbf{x} d \mathbf{y}=d x_{1} \wedge \cdots \wedge d x_{n} \wedge d y_{1} \wedge \cdots \wedge d y_{n}$ is $S_{n}$-invariant and thus defines a $2 n$-form on the smooth locus in $S^{n} \mathbb{C}^{2}$ and therefore a rational $2 n$-form on $H_{n}$.

If $I$ is a point of $U_{x}$, then $I$ is generated as an ideal in $R$ by two polynomials,

$$
x^{n}-e_{1} x^{n-1}+e_{2} x^{n-2}-\cdots+(-1)^{n} e_{n}
$$


and

$$
y-\left(a_{n-1} x^{n-1}+a_{n-2} x^{n-2}+\cdots+a_{0}\right),
$$

where the complex numbers $e_{1}, \ldots, e_{n}, a_{0}, \ldots, a_{n-1}$ are regular functions of $I$. This is so because the tautological sheaf $B$ is free with basis $1, x, \ldots, x^{n-1}$ on $U_{x}$, and the sections $x^{n}$ and $y$ must be unique linear combinations of the basis sections with regular coefficients. Conversely, for any choice of the parameters e, a, the polynomials in (30) and (31) generate an ideal $I \in U_{x}$. Hence $U_{x}$ is an affine $2 n$-cell with coordinates $\mathbf{e}, \mathbf{a}$.

On the open set where $\sigma(I)$ consists of points $P_{i}$ with distinct $x$ coordinates, the polynomial in (30) is $\prod_{i=1}^{n}\left(x-x_{i}\right)$. This implies that $e_{k}$ coincides as a rational function (and as a global regular function) with the $k$-th elementary symmetric function $e_{k}(\mathbf{x})$. Likewise, $a_{k}$ is given as a rational function of $\mathbf{x}$ and $\mathbf{y}$ by the coefficient of $x^{k}$ in the unique interpolating polynomial $\phi_{a}(x)$ of degree $n-1$ satisfying $\phi_{a}\left(x_{i}\right)=y_{i}$ for $i=1, \ldots, n$.

The equations $\phi_{a}\left(x_{i}\right)=y_{i}$ can be expressed as a matrix identity $\left(y_{1}, \ldots, y_{n}\right)=$ $\left(a_{0}, \ldots, a_{n-1}\right) M$, where $M$ is the Vandermonde matrix in the $\mathbf{x}$ variables. Modulo terms involving the $d x_{i}$, this yields the identity of rational $n$-forms on $H_{n}$,

$$
d \mathbf{a}=\Delta(\mathbf{x})^{-1} d \mathbf{y},
$$

where $\Delta(\mathbf{x})$ is the Vandermonde determinant. For the elementary symmetric functions $e_{k}=e_{k}(\mathbf{x})$ we have the well-known identity

$$
d \mathbf{e}=\Delta(\mathbf{x}) d \mathbf{x}
$$

Together these show that $d \mathbf{x} d \mathbf{y}=d \mathbf{a} d \mathbf{e}$ is a nowhere vanishing regular section of $\omega$ on $U_{x}$. By symmetry, the same holds on $U_{y}$. This shows that we have $\omega \cong \mathcal{O}$ on $U_{x} \cup U_{y}$ and hence everywhere, by Lemma 3.6.2

On $H_{n-1, n}$ we have two groups of twisting sheaves, $\mathcal{O}_{n-1}(k)$ and $\mathcal{O}_{n}(l)$, pulled back from $H_{n-1}$ and $H_{n}$ respectively. We abbreviate $\mathcal{O}(k, l)=\mathcal{O}_{n-1}(k) \otimes \mathcal{O}_{n}(l)$.

Proposition 3.6.4. The canonical sheaf $\omega_{H_{n-1, n}}$ on the nested Hilbert scheme $H_{n-1, n}$ is given by $\mathcal{O}(1,-1)$ in the above notation.

Proof. We have tautological sheaves $B_{n-1}$ and $B_{n}$ pulled back from $H_{n-1}$ and $H_{n}$. The kernel $L$ of the canonical surjection $B_{n} \rightarrow B_{n-1}$ is the line bundle with fiber $I_{n-1} / I_{n}$ at the point $\left(I_{n-1}, I_{n}\right)$. From Proposition 3.4 .3 we have $L=\mathcal{O}(-1,1)$. On the generic locus, the fiber $I_{n-1} / I_{n}$ can be identified with the one-dimensional space of functions on $V\left(I_{n}\right)$ that vanish except at $P_{n}$. Thus the ratio of two sections of $L$ is determined by evaluation at $x=x_{n}, y=y_{n}$.

Regarding the polynomials in (30) and (31) as regular functions on $U_{x} \times \mathbb{C}^{2}$, they are the defining equations of the universal family $F_{x}=\pi^{-1}\left(U_{x}\right)$ over $U_{x} \subseteq H_{n}$, as a closed subscheme of the affine scheme $U_{x} \times \mathbb{C}^{2}$. We can use these defining equations to eliminate $e_{n}$ and $y$, showing that $F_{x}$ is an affine cell with coordinates $x, e_{1}, \ldots, e_{n-1}, a_{0}, \ldots, a_{n-1}$.

Over the curvilinear locus, the morphism $\alpha: H_{n-1, n} \rightarrow F$ in (27) restricts to a bijective morphism of smooth schemes, hence an isomorphism. Under this isomorphism $x$ corresponds to the $x$-coordinate $x_{n}$ of the distinguished point, and modulo $x_{n}$ we can replace the elementary symmetric functions $e_{k}(\mathbf{x})$ with $e_{k}^{\prime}=e_{k}\left(x_{1}, \ldots, x_{n-1}\right)$, for $k=1, \ldots, n-1$. As in the proof of Proposition 3.6.3 
we now calculate that a nowhere vanishing regular section of $\omega$ on $U_{x} \subseteq H_{n-1, n}$ is given by

$$
t_{x}=d x_{n} d \mathbf{e}^{\prime} d \mathbf{a}=\frac{1}{\prod_{i=1}^{n-1}\left(x_{n}-x_{i}\right)} d \mathbf{x} d \mathbf{y} .
$$

By symmetry, $t_{y}=\left(1 / \prod_{i=1}^{n-1}\left(y_{n}-y_{i}\right)\right) d \mathbf{x} d \mathbf{y}$ is a nowhere vanishing regular section of $\omega$ on $U_{y}$.

Now, at every point of $U_{x}$, the ideal $I_{n-1}$ is generated modulo $I_{n}$ by

$$
x^{n-1}-e_{1}^{\prime} x^{n-2}+\cdots+(-1)^{n-1} e_{n-1}^{\prime}=\prod_{i=1}^{n-1}\left(x-x_{i}\right),
$$

so this expression represents a nowhere vanishing section $s_{x}$ of $L$ on $U_{x}$. Similarly, $\prod_{i=1}^{n-1}\left(y-y_{i}\right)$ represents a nowhere vanishing section $s_{y}$ of $L$ on $U_{y}$. By the observations in the first paragraph of the proof, the ratio $s_{x} / s_{y}$ is the rational function $\prod_{i=1}^{n-1}\left(x_{n}-x_{i}\right) / \prod_{i=1}^{n-1}\left(y_{n}-y_{i}\right)$ on $H_{n-1, n}$. Since we have nowhere vanishing sections $t_{x}, t_{y}$ of $\omega$ on $U_{x}$ and $U_{y}$ with $t_{y} / t_{x}=s_{x} / s_{y}$, it follows that we have $\omega \cong L^{-1}=\mathcal{O}(1,-1)$ on $U_{x} \cap U_{y}$ and hence everywhere, by Lemma 3.6 .2

3.7. Geometry of $X_{n}$ and the $n$ ! conjecture. Recall that the $n$ ! conjecture, Conjecture 2.2.1 concerns the space $D_{\mu}$ spanned by all derivatives of the polynomial $\Delta_{\mu}$ in $\$ 2.2$, eq. (11). Let

$$
J_{\mu}=\left\{p \in \mathbb{C}[\mathbf{x}, \mathbf{y}]: p(\partial \mathbf{x}, \partial \mathbf{y}) \Delta_{\mu}=0\right\}
$$

be the ideal of polynomials whose associated partial differential operators annihilate $\Delta_{\mu}$, and set

$$
R_{\mu}=\mathbb{C}[\mathbf{x}, \mathbf{y}] / J_{\mu} .
$$

Clearly $D_{\mu}$ and $R_{\mu}$ have the same dimension as vector spaces. The ideal $J_{\mu}$ is doubly homogeneous and $S_{n}$-invariant, so $R_{\mu}$ is a doubly graded ring with an action of $S_{n}$ which respects the grading.

We have the following useful characterization of the ideal $J_{\mu}$.

Proposition 3.7.1 ([24, Proposition 3.3). The ideal $J_{\mu}$ in $(36)$ is equal to the set of polynomials $p \in \mathbb{C}[\mathbf{x}, \mathbf{y}]$ such that the coefficient of $\Delta_{\mu}$ in $\Theta^{\epsilon}(g p)$ is zero for all $g \in \mathbb{C}[\mathbf{x}, \mathbf{y}]$.

Note that it makes sense to speak of the coefficient of $\Delta_{\mu}$ in an alternating polynomial, since the polynomials $\Delta_{D}$ form a basis of $\mathbb{C}[\mathbf{x}, \mathbf{y}]^{\epsilon}$.

It seems infeasible to describe explicitly the ideal sheaf of $X_{n}$ as a closed subscheme of $H_{n} \times\left(\mathbb{C}^{2}\right)^{n}$, but we can give an implicit description of this ideal sheaf by regarding $X_{n}$ as a closed subscheme of $F^{n}$. Here $F^{n}$ denotes the relative product of $n$ copies of the universal family $F$, as a scheme over $H_{n}$. Like $X_{n}, F^{n}$ is a closed subscheme of $H_{n} \times\left(\mathbb{C}^{2}\right)^{n}$. Its fiber over a point $I=I(S)$ is $S^{n}$, so the closed points of $F^{n}$ are the tuples $\left(I, P_{1}, \ldots, P_{n}\right)$ satisfying $P_{i} \in V(I)$ for all $i$. In particular $F^{n}$ contains $X_{n}$. Being a closed subscheme of $F^{n}, X_{n}$ can be defined as a scheme over $H_{n}$ by

$$
X_{n}=\operatorname{Spec} B^{\otimes n} / \mathcal{J}
$$

for some sheaf of ideals $\mathcal{J}$ in the sheaf of $\mathcal{O}_{H_{n}}$-algebras $B^{\otimes n}$. 
Proposition 3.7.2. Let

$$
B^{\otimes n} \otimes B^{\otimes n} \rightarrow B^{\otimes n} \rightarrow \wedge^{n} B
$$

be the map of $\mathcal{O}_{H_{n}}$-module sheaves given by multiplication, followed by the alternation operator $\Theta^{\epsilon}$. Then the ideal sheaf $\mathcal{J}$ of $X_{n}$ as a subscheme of $F^{n}$ is the kernel of the map

$$
\phi: B^{\otimes n} \rightarrow\left(B^{\otimes n}\right)^{*} \otimes \wedge^{n} B
$$

induced by (39)).

Proof. Let $U \subseteq H_{n}$ be the generic locus, the open set consisting of ideals $I \in$ $H_{n}$ for which $S=V(I)$ consists of $n$ distinct, reduced points. Note that $F^{n}$ is clearly reduced over $U$, and since $F^{n}$ is flat over $H_{n}$ and $U$ is dense, $F^{n}$ is reduced everywhere. (For this argument we do not have to assume that $F^{n}$ is irreducible, and indeed for $n>1$ it is not: $X_{n}$ is one of its irreducible components.) Sections of $B^{\otimes n}$ can be identified with regular functions on suitable open subsets of $F^{n}$. Since $X_{n}$ is reduced and irreducible (Proposition 3.3.2), the open set $W=\rho^{-1}(U)$ is dense in $X_{n}$, and $\mathcal{J}$ consists of those sections of $B^{\otimes n}$ whose restrictions to $U$ define regular functions vanishing on $W$.

Let $s$ be a section of $\mathcal{J}$. For any section $g$ of $B^{\otimes n}$, the section $\Theta^{\epsilon}(g s)$ also belongs to $\mathcal{J}$. Since it is alternating, $\Theta^{\epsilon}(g s)$ vanishes at every point $\left(I, P_{1}, \ldots, P_{n}\right) \in F^{n}$ for which two of the $P_{i}$ coincide. In particular $\Theta^{\epsilon}(g s)$ vanishes on $F^{n} \backslash X_{n}$ and hence on $F^{n}$, that is, $\Theta^{\epsilon}(g s)=0$. This is precisely the condition for $s$ to belong to the kernel of $\phi$.

Conversely, if $s$ does not vanish on $W$ there is a point $Q=\left(I, P_{1}, \ldots, P_{n}\right)$, with all $P_{i}$ distinct, where the regular function represented by $s$ is nonzero. Multiplying $s$ by a suitable $g$, we can arrange that $g s$ vanishes at every point in the $S_{n}$ orbit of $Q$, except for $Q$. Then $\Theta^{\epsilon}(g s) \neq 0$, so $s$ is not in the kernel of $\phi$.

Proposition 3.7.3. Let $Q_{\mu}$ be the unique point of $X_{n}$ lying over $I_{\mu} \in H_{n}$. The following are equivalent:

(1) $X_{n}$ is locally Cohen-Macaulay and Gorenstein at $Q_{\mu}$;

(2) the $n$ ! conjecture holds for the partition $\mu$.

When these conditions hold, moreover, the ideal of the scheme-theoretic fiber $\rho^{-1}\left(I_{\mu}\right)$ $\subseteq X_{\mu}$ as a closed subscheme of $\left(\mathbb{C}^{2}\right)^{n}$ coincides with the ideal $J_{\mu}$ in (36).

Proof. Since $B^{\otimes n}$ and $\left(B^{\otimes n}\right)^{*} \otimes \wedge^{n} B$ are locally free sheaves, the sheaf homomorphism $\phi$ in (40) can be identified with a linear homomorphism of vector bundles over $H_{n}$. Let

$$
\phi(I): B^{\otimes n}(I) \rightarrow B^{\otimes n}(I)^{*} \otimes \wedge^{n} B(I)
$$

denote the induced map on the fiber at $I$. The $\operatorname{rank} \operatorname{rk} \phi(I)$ of the fiber map is a lower semicontinuous function, that is, the set $\{I: \operatorname{rk} \phi(I) \geq r\}$ is open for all $r$. If $\operatorname{rk} \phi(I)$ is constant on an open set $U$, then the cokernel of $\phi$ is locally free on $U$, and conversely. When this holds, $\operatorname{im} \phi$ is also locally free, of rank equal to the constant value of $\operatorname{rk} \phi(I)$. By Proposition 3.7.2, we have $\operatorname{im} \phi=\rho_{*} \mathcal{O}_{X_{n}}$. The fiber of $X_{n}$ over a point $I$ in the generic locus consists of $n$ ! reduced points, so the generic rank of $\phi$ is $n !$. 
The fiber $B(I)$ of the tautological bundle at $I$ is $R / I$. Identifying $R^{\otimes n}$ with $\mathbb{C}[\mathbf{x}, \mathbf{y}]$, we have a linear map

$$
\eta: \mathbb{C}[\mathbf{x}, \mathbf{y}] \rightarrow B^{\otimes n}(I)^{*} \otimes \wedge^{n} B(I)
$$

given by composing $\phi(I)$ with the canonical map $\mathbb{C}[\mathbf{x}, \mathbf{y}] \rightarrow(R / I)^{\otimes n}$. It follows from the definition of $\phi$ that $\eta(p)=0$ if and only if $\lambda \Theta^{\epsilon}(g p)=0$ for all $g \in \mathbb{C}[\mathbf{x}, \mathbf{y}]$, where

$$
\lambda: A \rightarrow \wedge^{n}(R / I) \cong \mathbb{C}
$$

is the restriction of the canonical map $\mathbb{C}[\mathbf{x}, \mathbf{y}] \rightarrow(R / I)^{\otimes n}$ to $S_{n}$-alternating elements. In particular, for $I=I_{\mu}$, we have $\lambda\left(\Delta_{D}\right)=0$ for all $D \neq D(\mu)$, and $\lambda\left(\Delta_{\mu}\right)$ spans $\wedge^{n}(R / I)$. Hence, by Proposition 3.7.1, the kernel of $\eta$ is exactly the ideal $J_{\mu}$ in this case.

Suppose the $n$ ! conjecture holds for $\mu$. Then since $\eta$ and $\phi(I)$ have the same image, we have $\operatorname{rk} \phi\left(I_{\mu}\right)=n$ !. By lower semicontinuity, since $n$ ! is also the generic rank of $\phi, \operatorname{rk} \phi(I)$ is locally constant and equal to $n$ ! on a neighborhood of $I_{\mu}$. Hence coker $\phi$ and $\operatorname{im} \phi=\rho_{*} \mathcal{O}_{X_{n}}$ are locally free there. The local freeness of $\rho_{*} \mathcal{O}_{X_{n}}$ shows that $X_{n}$ is locally Cohen-Macaulay at $Q_{\mu}$.

Let $M$ be the maximal ideal of the regular local ring $\mathcal{O}_{H_{n}, I_{\mu}}$. Since $X_{n}$ is finite over $H_{n}$, the ideal $N=M \mathcal{O}_{X_{n}, Q_{\mu}}$ is a parameter ideal. Assuming the $n$ ! conjecture holds for $\mu$, the Cohen-Macaulay ring $\mathcal{O}_{X_{n}, Q_{\mu}}$ is Gorenstein if and only if $\mathcal{O}_{X_{n}, Q_{\mu}} / N$ is Gorenstein. We have $\mathcal{O}_{X_{n}, Q_{\mu}} / N \cong(\operatorname{im} \phi) \otimes_{\mathcal{O}_{H_{n}}} \mathcal{O}_{H_{n}, I_{\mu}} / M$. Factoring $\phi: B^{\otimes n} \rightarrow\left(B^{\otimes n}\right)^{*} \otimes \wedge^{n} B$ through $\operatorname{im} \phi$, then tensoring with $\mathcal{O}_{H_{n}, I_{\mu}} / M$, we see that the fiber map $\phi\left(I_{\mu}\right)$ factors as

$$
\phi\left(I_{\mu}\right): B^{\otimes n}\left(I_{\mu}\right) \rightarrow \mathcal{O}_{X_{n}, Q_{\mu}} / N \rightarrow B^{\otimes n}\left(I_{\mu}\right)^{*} \otimes \wedge^{n} B\left(I_{\mu}\right) .
$$

The first homomorphism above is surjective, and, since coker $\phi$ is locally free, the second homomorphism is injective. Hence $\mathcal{O}_{X_{n}, Q_{\mu}} / N$ is isomorphic to the image $\operatorname{im} \phi\left(I_{\mu}\right)=\operatorname{im} \eta \cong \mathbb{C}[\mathbf{x}, \mathbf{y}] / J_{\mu}$. This last ring is Gorenstein by [12], Proposition 4 .

Conversely, suppose $X_{n}$ is locally Gorenstein at $Q_{\mu}$. Then $\mathcal{O}_{X_{n}, Q_{\mu}} / N$ is a Gorenstein Artin local ring isomorphic to $\mathbb{C}[\mathbf{x}, \mathbf{y}] / J$ for some ideal $J$. Since $\rho_{*} \mathcal{O}_{X_{n}}$ is locally free on a neighborhood of $I_{\mu}$, necessarily of rank $n$ !, we have $\operatorname{dim}_{\mathbb{C}} \mathbb{C}[\mathbf{x}, \mathbf{y}] / J=$ $n$ !. The locally free sheaf $\rho_{*} \mathcal{O}_{X_{n}}$ is the sheaf of sections of a vector bundle, which is actually a bundle of $S_{n}$ modules, since $S_{n}$ acts on $X_{n}$ as a scheme over $H_{n}$. The isotypic components of such a bundle are direct summands of it and hence locally free themselves, so the character of $S_{n}$ on the fibers is constant. In our case, the generic fibers are the coordinate rings of the $S_{n}$ orbits of points $\left(P_{1}, \ldots, P_{n}\right) \in\left(\mathbb{C}^{2}\right)^{n}$ with all $P_{i}$ distinct. Therefore every fiber affords the regular representation of $S_{n}$.

The socle of $\mathbb{C}[\mathbf{x}, \mathbf{y}] / J$ is one-dimensional, by the Gorenstein property, and $S_{n}$-invariant. Since $\mathbb{C}[\mathbf{x}, \mathbf{y}] / J$ affords the regular representation, its only onedimensional invariant subspaces are $(\mathbb{C}[\mathbf{x}, \mathbf{y}] / J)^{S_{n}}$, which consists of the constants, and $(\mathbb{C}[\mathbf{x}, \mathbf{y}] / J)^{\epsilon}$. The socle must therefore be the latter space. The factorization of $\phi\left(I_{\mu}\right)$ in (44) implies that $J \subseteq J_{\mu}=\operatorname{ker} \eta$. If $J_{\mu} / J \neq 0$, then we must have $\operatorname{soc}(\mathbb{C}[\mathbf{x}, \mathbf{y}] / J) \subseteq J_{\mu} / J$, as the socle is contained in every nonzero ideal. But this would imply $\left(\mathbb{C}[\mathbf{x}, \mathbf{y}] / J_{\mu}\right)^{\epsilon}=0$ and hence $\Delta_{\mu} \in J_{\mu}$, which is absurd.

Proposition 3.7.4. If the $n$ ! conjecture holds for all partitions $\mu$ of a given $n$, then $X_{n}$ is Gorenstein with canonical line bundle $\omega_{X_{n}}=\mathcal{O}(-1)$. 
Proof. The set $U$ of points $I \in H_{n}$ such that $\operatorname{rk} \phi(I)=n$ ! is open and $\mathbb{T}^{2}$-invariant. From the proof of Proposition 3.7 .3 we see that the $n$ ! conjecture implies that $U$ contains all the monomial ideals $I_{\mu}$. Since every $I \in H_{n}$ has a monomial ideal in the closure of its orbit, this implies $U=H_{n}$, so $\rho: X_{n} \rightarrow H_{n}$ is flat, $X_{n}$ is CohenMacaulay, and $P=\rho_{*} \mathcal{O}_{X_{n}}$ is a locally free sheaf of rank $n$ !. By Proposition 3.7.2 the map in (39) induces a pairing

$$
P \otimes P \rightarrow \wedge^{n} B=\mathcal{O}(1)
$$

and $\phi$ factors through the induced homomorphism

$$
\tilde{\phi}: P \rightarrow P^{*} \otimes \mathcal{O}(1) \cong \mathcal{H o m}(P, \mathcal{O}(1)) .
$$

Note that $\mathcal{H o m}(P, \mathcal{O}(1))$ is a sheaf of $P$-modules, with multiplication by a section $s$ of $P$ given by $(s \lambda)(h)=\lambda(s h)$. By the definition of $\phi$ we have $\tilde{\phi}(s g)(h)=$ $\Theta^{\epsilon}(s g h)=\tilde{\phi}(g)(s h)$, so $\tilde{\phi}$ is a homomorphism of sheaves of $P$-modules. Since $\operatorname{rk} \phi(I)$ is constant and equal to $n$ !, which is the rank of both $P$ and $P^{*} \otimes \mathcal{O}(1), \tilde{\phi}$ is an isomorphism.

Now, $X_{n}=\operatorname{Spec} P$, so by the duality theorem, $\omega_{X_{n}}$ is the sheaf of $\mathcal{O}_{X_{n}}$-modules associated to the sheaf of $P$-modules $\omega_{H_{n}} \otimes P^{*}$. By Proposition 3.6.3 we have $\omega_{H_{n}} \cong \mathcal{O}_{H_{n}}$, and we have just shown $P^{*} \cong P \otimes \mathcal{O}(-1)$. Together these imply $\omega_{X_{n}}=\mathcal{O}_{X_{n}}(-1)$.

\subsection{Main theorem.}

Theorem 3.1. The isospectral Hilbert scheme $X_{n}$ is normal, Cohen-Macaulay, and Gorenstein, with canonical sheaf $\omega_{X_{n}} \cong \mathcal{O}(-1)$.

The proof of this theorem will occupy us for the rest of this subsection. In principle, to show that $X_{n}$ is Cohen-Macaulay (at a point $Q$, say), we would like to exhibit a local regular sequence of length $2 n=\operatorname{dim} X_{n}$. In practice, we are unable to do this, but we can show that the $\mathbf{y}$ coordinates form a regular sequence of length $n$ wherever they vanish. As it turns out, showing this much is half of the battle. A geometric induction argument takes care of the other half.

The key geometric property of $X_{n}$, which implies that the $\mathbf{y}$ coordinates form a regular sequence, is given by the following pair of results, the first of which is proven in 4.11

Proposition 3.8.1. Let $J=\mathbb{C}[\mathbf{x}, \mathbf{y}] A$ be the ideal generated by the space of alternating polynomials $A=\mathbb{C}[\mathbf{x}, \mathbf{y}]^{\epsilon}$. Then $J^{d}$ is a free $\mathbb{C}[\mathbf{y}]$-module for all $d$.

Corollary 3.8.2. The projection $X_{n} \rightarrow \mathbb{C}^{n}=\operatorname{Spec} \mathbb{C}[\mathbf{y}]$ of $X_{n}$ on the $\mathbf{y}$ coordinates is flat.

Proof. Let $S=\mathbb{C}[\mathbf{x}, \mathbf{y}]$. By Proposition [3.4.2, we have $X_{n}=\operatorname{Proj} S[t J]$, and Proposition 3.8.1 implies that $S[t J]$ is a free $\mathbb{C}[\mathbf{y}]$-module.

An alternating polynomial $g \in A$ must vanish at every point $\left(P_{1}, \ldots, P_{n}\right) \in$ $\left(\mathbb{C}^{2}\right)^{n}$ where two of the $P_{i}$ coincide. Hence we have

$$
J \subseteq \bigcap_{i<j}\left(x_{i}-x_{j}, y_{i}-y_{j}\right),
$$

and it is natural to conjecture by analogy to the univariate case that equality holds here. In [24], Proposition 6.2, we proved that the following more general identity holds once we know that $J^{d}$ is a free $\mathbb{C}[\mathbf{y}]$-module for all $n$ and $d$. 
Corollary 3.8.3. We have

$$
J^{d}=\bigcap_{i<j}\left(x_{i}-x_{j}, y_{i}-y_{j}\right)^{d}
$$

for all $n$ and $d$.

Remarkably, even though this seems like it should be an elementary result, we know of no proof not using Proposition 3.8.1, even for $d=1$. Using Corollary 3.8.3. we can deal with the normality question.

Proposition 3.8.4. The isospectral Hilbert scheme $X_{n}$ is arithmetically normal in its projective embedding over $\left(\mathbb{C}^{2}\right)^{n}$ as the blowup $X_{n}=\operatorname{Proj} S[t J]$, where $S=$ $\mathbb{C}[\mathbf{x}, \mathbf{y}]$. In particular $X_{n}$ is normal.

Proof. By definition arithmetically normal means that $S[t J]$ is a normal domain. Since $S$ itself is a normal domain, this is equivalent to the ideals $J^{d} \subseteq S$ being integrally closed ideals for all $d$. The powers of an ideal generated by a regular sequence are integrally closed, as is an intersection of integrally closed ideals, so $J^{d}$ is integrally closed by Corollary 3.8 .3

For the Cohen-Macaulay and Gorenstein properties of $X_{n}$ we use an inductive argument involving the nested Hilbert scheme, duality, and the following lemma.

Lemma 3.8.5. Let $g: Y \rightarrow X$ be a proper morphism. Let $z_{1}, \ldots, z_{m} \in \mathcal{O}_{X}(X)$ be global regular functions on $X$ (and, via $g$, on $Y$ ). Let $Z \subseteq X$ be the closed subset $Z=V\left(z_{1}, \ldots, z_{m}\right)$ and let $U=X \backslash Z$ be its complement. Suppose the following conditions hold.

(1) The $z_{i}$ form a regular sequence in the local ring $\mathcal{O}_{X, x}$ for all $x \in Z$.

(2) The $z_{i}$ form a regular sequence in the local ring $\mathcal{O}_{Y, y}$ for all $y \in g^{-1}(Z)$.

(3) Every fiber of $g$ has dimension less than $m-1$.

(4) The canonical homomorphism $\mathcal{O}_{X} \rightarrow R g_{*} \mathcal{O}_{Y}$ restricts to an isomorphism on $U$.

Then $\operatorname{Rg}_{*} \mathcal{O}_{Y}=\mathcal{O}_{X}$, i.e., the canonical homomorphism is an isomorphism.

Proof. The question is local on $X$, so without loss of generality we can assume $X=\operatorname{Spec} S$ is affine. Then we are to show that $H^{i}\left(Y, \mathcal{O}_{Y}\right)=0$ for $i>0$ and that $S \rightarrow H^{0}\left(Y, \mathcal{O}_{Y}\right)$ is an isomorphism.

Condition (3) implies that $H^{i}\left(Y, \mathcal{O}_{Y}\right)=0$ for $i \geq m-1$. Let $Z^{\prime}=g^{-1}(Z)$ and $U^{\prime}=g^{-1}(U)$. Conditions (1) and (2) imply that $\operatorname{depth}_{Z} \mathcal{O}_{X}$ and $\operatorname{depth}_{Z^{\prime}} \mathcal{O}_{Y}$ are both at least $m$. Hence the local cohomology modules $H_{Z}^{i}\left(\mathcal{O}_{X}\right)$ and $H_{Z^{\prime}}^{i}\left(\mathcal{O}_{Y}\right)$ vanish for $i \leq m-1$. By the exact sequence of local cohomology [26], we therefore have

$$
H^{i}\left(Y, \mathcal{O}_{Y}\right) \cong H^{i}\left(U^{\prime}, \mathcal{O}_{Y}\right)
$$

and

$$
H^{i}\left(X, \mathcal{O}_{X}\right) \cong H^{i}\left(U, \mathcal{O}_{X}\right)
$$

for all $i<m-1$. Condition (4) yields $H^{i}\left(U^{\prime}, \mathcal{O}_{Y}\right) \cong H^{i}\left(U, \mathcal{O}_{X}\right)$. Thus we have $H^{i}\left(Y, \mathcal{O}_{Y}\right) \cong H^{i}\left(X, \mathcal{O}_{X}\right)$ for all $i<m-1$.

Since $X$ is affine, this shows $H^{i}\left(Y, \mathcal{O}_{Y}\right)=0$ for all $i>0$, and $S \cong H^{0}\left(Y, \mathcal{O}_{Y}\right)$. The isomorphism $S \cong H^{0}\left(Y, \mathcal{O}_{Y}\right)$ is the canonical homomorphism, since it is determined by its restriction to $U$. 
We now prove Theorem 3.1 by induction on $n$. In the proof of the inductive step we will assume that $n>3$, so we must first dispose of the cases $n=1,2,3$. By Proposition 3.7.4, the theorem is equivalent to the $n$ ! conjecture, which can be verified for $n \leq 3$ by a simple calculation. Our induction hypothesis will be the statement of the theorem, plus the assertion that the projection $g: X_{n-1, n} \rightarrow X_{n}$ satisfies $R g_{*} \mathcal{O}_{X_{n-1, n}}=\mathcal{O}_{X_{n}}$. Therefore we also need to verify the latter fact for $n \leq 3$.

For $n=1$, we have $X_{0,1}=X_{1}=\mathbb{C}^{2}$ trivially, and for $n=2, X_{1,2}=X_{2}$ is the blowup of $\left(\mathbb{C}^{2}\right)^{2}$ along the diagonal. In these cases $g$ is an isomorphism. Note that $X_{1}$ and $X_{2}$ are actually nonsingular. Up to automorphisms induced by translations in $\mathbb{C}^{2}$, the scheme $X_{3}$ has an essentially isolated singularity at the point $Q_{(2,1)}$ lying over $I_{(2,1)} \in H_{n}$. We can fix a representative point in each translation class by restricting attention to the loci $\hat{X}_{2,3} \subseteq X_{2,3}$ and $\hat{X}_{3} \subseteq X_{3}$ defined by the vanishing of $x_{3}$ and $y_{3}$. Then $X_{2,3} \cong \hat{X}_{2,3} \times \mathbb{C}^{2}$ and $X_{3} \cong \hat{X}_{3} \times \mathbb{C}^{2}$, so we only need to consider the morphism $\hat{g}: \hat{X}_{2,3} \rightarrow \hat{X}_{3}$. We have $\operatorname{dim} \hat{X}_{2,3}=\operatorname{dim} \hat{X}_{3}=4$. The fiber of $\hat{g}$ over the unique singular point $Q_{(2,1)} \in \hat{X}_{3}$ is a projective line, and $\hat{g}$ is one-to-one on the complement of this fiber. Let $z_{1}, z_{2}, z_{3}$ be part of a system of local parameters on $\hat{X}_{3}$ at $Q_{(2,1)}$, so the locus $Z=V(\mathbf{z}) \subseteq \hat{X}_{3}$ has dimension 1. The locus $\hat{g}^{-1}(Z) \subseteq \hat{X}_{2,3}$ is the union of the fiber over $Q_{(2,1)}$ and the preimage of $Z \backslash\left\{Q_{(2,1)}\right\}$. Hence it also has dimension 1. Now $\hat{X}_{3}$ is Cohen-Macaulay by Theorem 3.1 for $n=3$, and $\hat{X}_{2,3}$ is Cohen-Macaulay by the theorem for $n=2$ and the argument which will be given below for general $n$. The hypotheses of Lemma 3.8.5 are satisfied, so we have $R \hat{g}_{*} \mathcal{O}_{\hat{X}_{2,3}}=\mathcal{O}_{\hat{X}_{3}}$.

Assume now by induction that $X_{n-1}$ is Cohen-Macaulay and Gorenstein with $\omega_{X_{n-1}}=\mathcal{O}_{X_{n-1}}(-1)$. Then $\rho_{n-1}: X_{n-1} \rightarrow H_{n-1}$ is flat and finite with Gorenstein fibers. In the scheme-theoretic fiber square

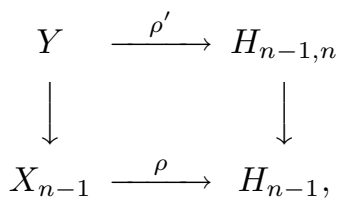

the morphism $\rho^{\prime}$ is therefore also flat and finite with Gorenstein fibers. Since $H_{n-1, n}$ is nonsingular, $Y$ is Cohen-Macaulay and Gorenstein. On the generic locus, where $P_{1}, \ldots, P_{n}$ are all distinct, the above diagram coincides locally with the fiber square

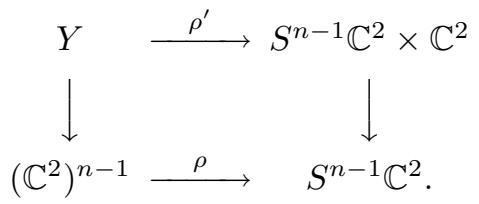

This shows that $Y$ is generically reduced, hence reduced, as well as irreducible and birational to $\left(\mathbb{C}^{2}\right)^{n}$. The reduced fiber product in (51) is $X_{n-1, n}$ by definition, so we have $Y=X_{n-1, n}$. Thus $X_{n-1, n}$ is Cohen-Macaulay and Gorenstein. Furthermore, the relative canonical sheaf of $X_{n-1, n}$ over $H_{n-1, n}$ is the pullback of that of $X_{n-1}$ over $H_{n-1}$. By Proposition 3.6.3 and the induction hypothesis, the latter is $\mathcal{O}_{X_{n-1}}(-1)$ and its pullback to $X_{n-1, n}$ is $\mathcal{O}(-1,0)$. By Proposition 3.6.4 it follows that the canonical sheaf on $X_{n-1, n}$ is $\omega_{X_{n-1, n}}=\mathcal{O}(-1,0) \otimes \mathcal{O}(1,-1)=\mathcal{O}(0,-1)$. 
Now consider the projection

$$
g: Y=X_{n-1, n} \rightarrow X_{n} .
$$

We claim that $R g_{*} \mathcal{O}_{Y}=\mathcal{O}_{X_{n}}$. By the projection formula, since $\mathcal{O}(0,-1)=$ $g^{*} \mathcal{O}_{X_{n}}(-1)$ is pulled back from $X_{n}$, this implies also $R g_{*} \mathcal{O}(0,-1)=\mathcal{O}_{X_{n}}(-1)$. Now $\mathcal{O}(0,-1)[2 n]=\omega_{X_{n-1, n}}[2 n]$ is the dualizing complex on $X_{n-1, n}$, so by the duality theorem it follows that $\mathcal{O}(-1)[2 n]$ is the dualizing complex on $X_{n}$. In other words, $X_{n}$ is Gorenstein, with canonical sheaf $\omega_{X_{n}}=\mathcal{O}(-1)$, which is what we wanted to prove.

For the claim, we verify the conditions of Lemma 3.8.5 with $Y=X_{n-1, n}, X=$ $X_{n}$, and $z_{1}, \ldots, z_{n-1}$ equal to $y_{1}-y_{2}, \ldots, y_{n-1}-y_{n}$. For the $z_{i}$ to form a regular sequence it suffices that $y_{1}, \ldots, y_{n}$ is a regular sequence. Strictly speaking, this only shows that the $z_{i}$ form a regular sequence where the $y_{i}$ are all equal to zero. However, since all automorphisms of $\mathbb{C}^{2}$, and translations in the $y$ direction in particular, act on all schemes under consideration, it follows that the $z_{i}$ form a regular sequence wherever the $y_{i}$ are all equal to each other, that is, on $V(\mathbf{z})$.

On $Y=X_{n-1, n}$ the fact that the $y_{i}$ form a regular sequence follows from the Cohen-Macaulay property of $X_{n-1, n}$, together with Proposition 3.5.6. which says that $V(\mathbf{y})$ is a complete intersection in $Y$. On $X=X_{n}$ (this is the crucial step!), the regular sequence condition follows from Corollary 3.8 .2

On the open set $U=X_{n} \backslash V(\mathbf{z})$, the coordinates $y_{i}$ are nowhere all equal. It follows from Lemmas 3.3.1 and 3.5.5 that $U$ can be covered by open sets on which the projection $g: X_{n-1, n} \rightarrow X_{n}$ is locally isomorphic to $1 \times g_{l}: X_{k} \times X_{l-1, l} \rightarrow$ $X_{k} \times X_{l}$ for some $k+l=n$ with $l<n$. We can assume as part of the induction that $R\left(g_{l}\right)_{*} \mathcal{O}_{X_{l-1, l}}=\mathcal{O}_{X_{l}}$. Hence we have $\left.R g_{*} \mathcal{O}_{X_{n-1, n}}\right|_{g^{-1}(U)}=\left.\mathcal{O}_{X_{n}}\right|_{U}$.

For the fiber dimension condition, note that the fiber of $g$ over a point $\left(I, P_{1}, \ldots, P_{n}\right)$ of $X_{n}$ is the same as the fiber of the morphism $\alpha$ in (27) over $\left(I, P_{n}\right)$. By Proposition 3.5.3, its dimension $d$ satisfies the inequality $\left(\begin{array}{c}d+2 \\ 2\end{array}\right) \leq n$. Since we are assuming $n>3$, this inequality easily implies $d<n-2$, as required for Lemma 3.8.5 to hold.

3.9. Proof of the graded character conjecture. We now review in outline the proof from 24] that the $n$ ! conjecture and the Cohen-Macaulay property of $X_{n}$ imply Conjecture 2.2.2 The proof involves some technical manipulations with Frobenius series and Macdonald polynomials which it would take us too far afield to repeat in full here. Conceptually, however, the argument is straightforward. The main point is the connection between $S_{n}$ characters and symmetric functions given by the Frobenius characteristic

$$
\Psi(\chi)=\frac{1}{n !} \sum_{w \in S_{n}} \chi(w) p_{\tau(w)}(x)
$$

Here $p_{\tau}(x)$ denotes a power-sum symmetric function and $\tau(w)$ is the partition of $n$ given by the cycle lengths in the expression for $w$ as a product of disjoint cycles. The Frobenius characteristics of the irreducible characters are the Schur functions (40], I, eq. (7.5))

$$
\Psi\left(\chi^{\lambda}\right)=s_{\lambda}(x)
$$

Let $J_{\mu}$ be the ideal of operators annihilating $\Delta_{\mu}$, and set $R_{\mu}=\mathbb{C}[\mathbf{x}, \mathbf{y}] / J_{\mu}$, as in (36) - (37). It follows from [24], Proposition 3.4, that $\operatorname{ch}\left(R_{\mu}\right)_{r, s}=\operatorname{ch}\left(D_{\mu}\right)_{r, s}$ for all degrees $r, s$, so we may replace $D_{\mu}$ with $R_{\mu}$ in the statement of Conjecture 2.2.2 
We define the Frobenius series of $R_{\mu}$ to be

$$
\mathcal{F}_{R_{\mu}}(x ; q, t)=\sum_{r, s} t^{r} q^{s} \Psi\left(\operatorname{ch}\left(R_{\mu}\right)_{r, s}\right)
$$

The Frobenius series is a kind of doubly graded Hilbert series that keeps track of characters instead of just dimensions. In this notation Conjecture 2.2.2 takes the form of an identity

$$
\mathcal{F}_{R_{\mu}}(x ; q, t)=\tilde{H}_{\mu}(x ; q, t) .
$$

There is a well-defined formal extension of the notion of Frobenius series to certain local rings with $S_{n}$ and $\mathbb{T}^{2}$ actions, as explained in 24], §5. In particular, we can define $\mathcal{F}_{S_{\mu}}(x ; q, t)$, where $S_{\mu}=\mathcal{O}_{X_{n}, Q_{\mu}}$ is the local ring of $X_{n}$ at the distinguished point $Q_{\mu}$ lying over $I_{\mu}$.

By Theorem 3.1 and Proposition 3.7.3, the ring $R_{\mu}$ is the coordinate ring of the scheme-theoretic fiber $\rho^{-1}\left(I_{\mu}\right) \subseteq X_{n}$. This and the flatness of $\rho$ imply ([24], eq. (5.3)) that the Frobenius series of $S_{\mu}$ and $R_{\mu}$ are related by a scalar factor,

$$
\mathcal{F}_{S_{\mu}}(x ; q, t)=\mathcal{H}(q, t) \mathcal{F}_{R_{\mu}}(x ; q, t),
$$

where $\mathcal{H}(q, t)$ is the formal Hilbert series of the local ring $\mathcal{O}_{H_{n}, I_{\mu}}$, as defined in [24], a rational function of $q$ and $t$. In fact, it follows from the determination in [23] of explicit regular local parameters on $H_{n}$ at $I_{\mu}$ that $\mathcal{H}(q, t)$ is the reciprocal of the polynomial

$$
\prod_{x \in D(\mu)}\left(1-q^{-a(x)} t^{1+l(x)}\right) \cdot \prod_{x \in D(\mu)}\left(1-q^{1+a(x)} t^{-l(x)}\right),
$$

where $a(x)$ and $l(x)$ denote the lengths of the arm and leg of the cell $x$ in the diagram of $\mu$. It is interesting to note that the two products above are essentially the normalizing factors introduced in [40], VI, eqs. (8.1-8.1'), to define the integral form Macdonald polynomials $J_{\mu}$. This coincidence is typical of the numerological parallels that already pointed to a link between Macdonald polynomials and the Hilbert scheme, before the theory presented here was fully developed.

Now, the global regular functions $y_{1}, \ldots, y_{n}$ form a regular sequence in the local ring $S_{\mu}$, as follows from the proof of Theorem 3.1] By [24], Proposition 5.3, part (3), this implies that

$$
\mathcal{F}_{S_{\mu} /(\mathbf{y})}(x ; q, t)=\mathcal{F}_{S_{\mu}}[X(1-q) ; q, t] .
$$

Furthermore, 24, Proposition 5.3, part (1), implies that if the irreducible character $\chi^{\lambda}$ has multiplicity zero in $\operatorname{ch} R_{\mu} /(\mathbf{y})$, then the coefficient of the Schur function $s_{\lambda}$ in $\mathcal{F}_{S_{\mu} /(\mathbf{y})}(x ; q, t)$ is equal to zero.

The ring $R_{\mu} /(\mathbf{y})$, which is simply the $y$-degree zero part of $R_{\mu}$, is a wellunderstood algebraic entity. By the results of [4, 17, it is isomorphic to the cohomology ring of the Springer fiber over a unipotent element of GL $(n)$, as defined in 45]. Its Frobenius series, which is the $q=0$ specialization $\mathcal{F}_{R_{\mu}}(x ; 0, t)$, is given by the transformed Hall-Littlewood polynomial

$$
\mathcal{F}_{R_{\mu}}(x ; 0, t)=t^{n(\mu)} Q_{\mu}\left[X /\left(1-t^{-1}\right) ; t^{-1}\right],
$$

where $Q_{\mu}(x ; t)$ is defined as in 40, III, eq. (2.11). We remark that (61) is just the $q=0$ specialization of (57). Equation (61) implies that the only characters $\chi^{\lambda}$ that 
occur with nonzero multiplicity in $\operatorname{ch} R_{\mu} /(\mathbf{y})$ are those with $\lambda \geq \mu$. Hence by (58) and (60) we have

$$
\mathcal{F}_{R_{\mu}}[X(1-q) ; q, t] \in \mathbb{Q}(q, t)\left\{s_{\lambda}(x): \lambda \geq \mu\right\} .
$$

This shows that $\mathcal{F}_{R_{\mu}}(x ; q, t)$ satisfies condition (1) of the characterization of $\tilde{H}_{\mu}(x ; q, t)$ in Proposition 2.1.1. By the symmetry between $\mathbf{x}$ and $\mathbf{y}, \mathcal{F}_{R_{\mu}}$ also satisfies condition (2). Finally, condition (3) for $\mathcal{F}_{R_{\mu}}$ says that the trivial character occurs only in degree zero, with multiplicity one. This is obvious, since the only $S_{n}$-invariants in $R_{\mu}$ are the constants. Hence (57) holds.

To summarize, we have the following consequence of Theorem 3.1 and Proposition 3.7 .3

Theorem 3.2. Conjectures 2.1.2, 2.2.1 and [2.2.2, that is, the Macdonald positivity conjecture, the $n$ ! conjecture and the graded character interpretation of $\tilde{K}_{\lambda \mu}(q, t)$, are all true.

3.10. Appendix: Cohen-Macaulay and Gorenstein schemes and duality theory. Here we give a synopsis of duality theory as used in this paper. Since we are chiefly interested in the connection between duality and the theory of CohenMacaulay and Gorenstein singularities, we formulate the theory in terms of dualizing complexes. The formulation below is valid for schemes $X$ of finite type over a field $k$, which is general enough for our purposes.

Let $D(X)$ denote the derived category of complexes of sheaves of $\mathcal{O}_{X}$-modules with bounded, coherent cohomology. If $A$ is a coherent sheaf, we denote by $A[d]$ the complex which is $A$ in degree $-d$ and zero in every other degree. If $A^{\bullet}$ is any complex in $D(X)$ whose only nonzero cohomology sheaf is $H^{-d}\left(A^{\bullet}\right) \cong A$, then we have $A^{\bullet} \cong A[d]$. We say that such a complex $A^{\bullet}$ reduces to a sheaf.

If a complex $\omega^{\bullet} \in D(X)$ has finite injective dimension, then the functor $\mathcal{D}_{\omega} \bullet=$ $R \mathcal{H} \operatorname{om}\left(-, \omega^{\bullet}\right)$ carries $D(X)$ into itself. A dualizing complex on $X$ is a complex $\omega^{\bullet}$ of finite injective dimension such that the canonical natural transformation $1_{D(X)} \rightarrow \mathcal{D}_{\omega} \bullet \circ \mathcal{D}_{\omega} \bullet$ is an isomorphism (see [25], $\mathrm{V}, \S 2$ ).

There is a preferred choice of dualizing complex $\omega_{X}^{\bullet}$ on each scheme $X$ of finite type over $k$, defined by $\omega_{X}^{\bullet}=f^{!} \mathcal{O}$, where $f: X \rightarrow$ Spec $k$ is the defining morphism of $X$ as a scheme over $k$. The functor $f^{!}$here is the one given by [25], VII, Corollary 3.4. That $\omega_{X}^{\bullet}$ is a dualizing complex follows from the definition of $f^{!}$given there, together with the fact that $\mathcal{O}$ is a dualizing complex on $X=\operatorname{Spec} k$. With this choice, the corresponding dualizing functors $\mathcal{D}_{X}=\mathcal{D}_{\omega_{X}}$ have the following properties.

(1) Duality theorem ([25], VII, Theorem 3.3): If $f: Y \rightarrow X$ is a proper morphism, then there is a canonical natural isomorphism

$$
\mathcal{D}_{X} \circ R f_{*} \cong R f_{*} \circ \mathcal{D}_{Y} \text {. }
$$

(2) If $f: Y \rightarrow X$ is smooth of relative dimension $d$, then $\omega_{Y}^{\bullet}=\omega_{Y / X}[d] \otimes f^{*} \omega_{X}^{\bullet}$, where $\omega_{Y / X}$ is the relative canonical line bundle, that is, the sheaf of relative exterior $d$-forms $\Omega_{Y / X}^{d}$. This follows from the definition of $f^{\sharp}$ in [25], III, $\S 2$, and the fact that $f^{!}=f^{\sharp}$ for a smooth morphism $f$.

A scheme $X$ is Cohen-Macaulay (respectively, Gorenstein) if its local ring $\mathcal{O}_{X, x}$ at every point is a Cohen-Macaulay (or Gorenstein) local ring. Since a localization of a Cohen-Macaulay or a Gorenstein ring is again Cohen-Macaulay or Gorenstein, it suffices that the condition hold for closed points $x$. If $\rho: X \rightarrow H$ is a finite morphism of equidimensional schemes of the same dimension, with $H$ nonsingular, 
then $X$ is Cohen-Macaulay if and only if $\rho$ is flat. When these conditions hold, $X$ is also Gorenstein if and only if the scheme-theoretic fibers of $\rho$ are Gorenstein.

The Cohen-Macaulay property has a simple characterization in terms of the dualizing complex. Recall that if $A$ is a Noetherian local ring of dimension $d$ and $x \in \operatorname{Spec} A$ is the closed point (the maximal ideal of $A$ ), then $A$ is Cohen-Macaulay if and only if the local cohomology modules $H_{x}^{i}(A)$ are zero except for $i=d$. Let $x$ be a closed point of a scheme $X$ of finite type over $k$ and let $A=\mathcal{O}_{X, x}$ be the local ring at $x$. By 25, $\mathrm{V}$, Corollary $2.3, \Omega^{\bullet}=\omega_{X, x}^{\bullet}$ is a dualizing complex on $\operatorname{Spec} A$. The duality theorem for the closed embedding of the point $x$ in $X$ implies that $\Omega^{\bullet}$ is a normalized dualizing complex in the sense of [25], $\mathrm{V}, \S 6$. It now follows from [25], V, Corollary 6.3, that $X$ is Cohen-Macaulay if and only if $\omega_{X}^{\bullet}$ reduces to a sheaf, concentrated in degree $-d$ on all connected components of $X$ with dimension $d$. Note that if $\omega_{X}^{\bullet}$ reduces to a sheaf on each connected component of $X$, then it must be concentrated in the correct degree, since any scheme $X$ of finite type over $k$ is generically Cohen-Macaulay.

Recall that if $A$ is a Cohen-Macaulay local ring of dimension $d$, then its canonical module $\Omega$ is the Matlis dual $\Omega \cong \operatorname{Hom}\left(H_{x}^{d}(A), I\right)$ of the $d$-th local cohomology module, where $I$ is an injective hull of the residue field $A / x$. Therefore it further follows from [25], V, Corollary 6.3, that if $X$ is Cohen-Macaulay, then the stalk $\omega_{X, x}^{\bullet}$ of the dualizing complex is isomorphic to $\Omega[d]$, where $\Omega$ is the canonical module of $A=\mathcal{O}_{X, x}$. In particular, $X$ is Gorenstein if and only if $\omega_{X}^{\bullet}$ reduces to a line bundle, i.e., a locally free sheaf of rank 1 , on each connected component of $X$. More precisely, when $X$ is Gorenstein, $\omega_{X}^{\bullet}$ reduces to $\omega_{X}[d]$ on components of dimension $d$, where $\omega_{X}$ is the canonical line bundle.

\section{Polygraphs}

4.1. First definitions. Let $E=\mathbb{A}^{2}(k)$ be the affine plane over a field $k$ of characteristic zero (the restriction on $k$ is not really necessary - see 4.12). We are going to study certain unions of linear subspaces, or subspace arrangements, in $E^{n} \times E^{l}$. We call these arrangements polygraphs because their constituent subspaces are the graphs of linear maps from $E^{n}$ to $E^{l}$.

Let $[n]$ denote the set of integers $\{1, \ldots, n\}$. Given a function $f:[l] \rightarrow[n]$, there is a linear morphism

$$
\pi_{f}: E^{n} \rightarrow E^{l}
$$

defined by

$$
\pi_{f}\left(P_{1}, \ldots, P_{n}\right)=\left(P_{f(1)}, \ldots, P_{f(l)}\right) .
$$

Let

$$
W_{f} \subseteq E^{n} \times E^{l}
$$

be the graph of $\pi_{f}$. We denote the coordinates on $E^{n} \times E^{l}$ by

$$
\mathbf{x}, \mathbf{y}, \mathbf{a}, \mathbf{b}=x_{1}, y_{1}, \ldots, x_{n}, y_{n}, a_{1}, b_{1}, \ldots, a_{l}, b_{l},
$$

where $x_{j}, y_{j}$ are the coordinates on the $j$-th factor in $E^{n}$ and $a_{i}, b_{i}$ are the coordinates on the $i$-th factor in $E^{l}$. In coordinates, $W_{f}$ is then defined by the equations

$$
W_{f}=V\left(I_{f}\right), \quad \text { where } \quad I_{f}=\sum_{i \in[l]}\left(a_{i}-x_{f(i)}, b_{i}-y_{f(i)}\right) .
$$


Definition 4.1.1. The polygraph $Z(n, l) \subseteq E^{n} \times E^{l}$ is the subspace arrangement

$$
Z(n, l)=\bigcup W_{f}, \quad \text { over all } f:[l] \rightarrow[n] .
$$

The geometric points of $Z(n, l)$ are the points $(\mathbf{x}, \mathbf{y}, \mathbf{a}, \mathbf{b})=\left(P_{1}, \ldots, P_{n}\right.$, $\left.Q_{1}, \ldots, Q_{l}\right)$ satisfying the following condition: for all $i \in[l]$ there is a $j \in[n]$ such that $Q_{i}=\left(a_{i}, b_{i}\right)$ is equal to $P_{j}=\left(x_{j}, y_{j}\right)$. Note that for $l=0$, the polygraph $Z(n, 0)$ makes sense and is equal to $E^{n}$. The index set $[l]=[0]$ is empty in this case, and the unique function $f:[0] \rightarrow[n]$ is the empty function $f=\emptyset$, with $I_{\emptyset}=0$ and $W_{\emptyset}=E^{n}$.

A word on terminology is in order here. For clarity it is often useful to describe a scheme or a morphism in terms of geometric points, that is, points defined by values of the coordinates in some algebraically closed extension $K$ of $k$. In the preceding paragraph and in (64), we have given geometric descriptions. In all other contexts, however, we will use the term point in the scheme-theoretic sense. When discussing the local geometry of $Z(n, l)$ at a point $P$, for instance, we mean that $P \in \operatorname{Spec} R(n, l)$ is a prime ideal in the coordinate $\operatorname{ring} R(n, l)$ of $Z(n, l)$. Since all schemes under consideration are closed subschemes of $E^{n} \times E^{l}$, we can also identify $P$ with a prime ideal of $k[\mathbf{x}, \mathbf{y}, \mathbf{a}, \mathbf{b}]$. Ultimately, everything we do reduces to commutative algebra and ideal theory in the polynomial ring $k[\mathbf{x}, \mathbf{y}, \mathbf{a}, \mathbf{b}]$. Any geometric descriptions we give are best understood merely as guides to formal definitions in terms of ideals and ring homomorphisms. Thus the polygraph $Z(n, l)$ is correctly defined as the subscheme of $E^{n} \times E^{l}$ whose ideal is the intersection of the ideals $I_{f}$ defined in (67).

Our purpose in this section is to prove the following theorem.

Theorem 4.1. The coordinate ring $R(n, l)=\mathcal{O}(Z(n, l))$ of the polygraph $Z(n, l)$ is a free $k[\mathbf{y}]$-module.

4.2. Examples. As motivation for Theorem 4.1, it may be helpful to consider the case of polygraphs in one set of variables, that is, the subspace arrangements defined as in 4.1.1, but with $E=\mathbb{A}^{1}$.

Polygraphs as we have defined them are in two sets of variables, namely the $\mathbf{x}, \mathbf{a}$ and the $\mathbf{y}, \mathbf{b}$. Had we begun with $E=\mathbb{A}^{d}$ instead of $E=\mathbb{A}^{2}$, we would have $d$ sets of variables (and notational headaches galore). In one set of variables the coordinates are just $\mathbf{x}, \mathbf{a}$, and the ideal of $Z(n, l)$ is simply

$$
I=\sum_{i \in[l]}\left(\prod_{j \in[n]}\left(a_{i}-x_{j}\right)\right) .
$$

Indeed, $I$ clearly defines $Z(n, l)$ set-theoretically, and it is a complete intersection ideal, since $Z(n, l)$ has codimension $l$, while $I$ has $l$ generators. It is easy to see that $V(I)$ is generically reduced: if $P$ is a point where the $x_{j}$ are all distinct, then only one $W_{f}$ passes through $P$, and each factor $\left(a_{i}-x_{j}\right)$ in (69) with $j \neq f(i)$ is a unit in the local ring $k[\mathbf{x}, \mathbf{a}]_{P}$, so $I_{P}$ coincides with the local ideal $\left(I_{f}\right)_{P}$. A generically reduced complete intersection is reduced, so $I=I(Z(n, l))$.

Now $R(n, l)=\mathcal{O}(Z(n, l))$ is a complete intersection ring, hence Cohen-Macaulay, and since $Z(n, l)$ is finite over $E^{n}$, the variables $\mathbf{x}$ form a homogeneous system of parameters. This implies that $R(n, l)$ is a free $k[\mathbf{x}]$-module. In fact, it is free with basis consisting of monomials $\mathbf{a}^{e}=a_{1}^{e_{1}} \cdots a_{l}^{e_{l}}, 0 \leq e_{i}<n$, since these monomials span modulo $I$ and their number is equal to $n^{l}$, which is the number of $W_{f}$ 's and thus the degree of the finite flat morphism $Z(n, l) \rightarrow E^{n}$. 
Returning to polygraphs in two sets of variables, the analog of (69), namely

$$
I=\sum_{i \in[l]} \prod_{j \in[n]}\left(a_{i}-x_{j}, b_{i}-y_{j}\right),
$$

clearly defines $Z(n, l)$ set-theoretically, but now it is not a complete intersection ideal, and does not define $Z(n, l)$ as a reduced scheme, that is, $I \neq \sqrt{ } I$. At present, we do not have a good conjecture as to a set of generators for the full ideal $I(Z(n, l))$ in general. To get a feeling for the possibilities, the reader might look ahead to \$4.6 eq. (96), where generators for the ideal are given in the case $n=2$.

In the bivariate situation, $Z(n, l)$ is not Cohen-Macaulay. In fact, $Z(2,1)$ is isomorphic to $E \times Y$, where $Y$ is the union of two linear 2-spaces in $\mathbb{A}^{4}$ that meet only at the origin. This $Y$ is the classic simplest example of an equidimensional affine algebraic set whose coordinate ring is not Cohen-Macaulay (and also not a complete intersection, even set-theoretically).

The property that does extend from the univariate case, according to Theorem 4.1 is that $R(n, l)$ is free over the ring of polynomials in one set of the $\mathbf{x}, \mathbf{y}$ variables, which by symmetry we have taken without loss of generality to be $\mathbf{y}$. We expect the analog of Theorem 4.1 to hold in $d$ sets of variables, with freeness over one set of the variables (Conjecture 5.2.2). The proof given here, however, is specific to the bivariate case.

As a further example, let us consider the case $l=1$. We will give a simple proof of Theorem 4.1 for $Z(n, 1)$, which also works in $d$ sets of variables after some obvious modifications.

For $l=1$, we write $W_{j}$ instead of $W_{f}$, where $j=f(1)$. The subspaces $W_{j}=$ $V\left(a_{1}-x_{j}, b_{1}-y_{j}\right)$ meet transversely, since a change of variables to $x_{j}^{\prime}=x_{j}-a_{1}$, $y_{j}^{\prime}=y_{j}-b_{1}$ makes them into coordinate subspaces. It follows that the ideal $I(Z(n, 1))$ of their union is the product of the ideals $I_{j}$. In other words, for $l=1$, the ideal $I(Z(n, 1))$ is equal to the ideal in (70) .

Now $W_{n}$ projects isomorphically on $E^{n}$, the projection being given in coordinates by the substitutions $a_{1} \mapsto x_{n}, b_{1} \mapsto y_{n}$. These substitutions carry the ideal of $W_{1} \cup \cdots \cup W_{n-1}$ to

$$
\prod_{j=1}^{n-1}\left(x_{n}-x_{j}, y_{n}-y_{j}\right)
$$

This shows that the scheme-theoretic intersection of $W_{n}$ with $W_{1} \cup \cdots \cup W_{n-1}$ is isomorphic to $Z(n-1,1)$, the coordinates $a_{1}, b_{1}$ on $Z(n-1,1)$ being identified with $x_{n}, y_{n}$. Since $W_{1} \cup \cdots \cup W_{n-1}=Z(n-1,1) \times E$, we have an exact sequence of $k\left[\mathbf{x}, \mathbf{y}, a_{1}, b_{1}\right]$-modules

$$
0 \rightarrow R(n, 1) \rightarrow k[\mathbf{x}, \mathbf{y}] \oplus\left(R(n-1,1) \otimes k\left[x_{n}, y_{n}\right]\right) \rightarrow R(n-1,1) \rightarrow 0 .
$$

The middle term here is the direct sum of the coordinate rings of $W_{n}$ and $W_{1} \cup \cdots \cup$ $W_{n-1}$; the outer terms are the coordinate rings of their union and their schemetheoretic intersection. By induction on $n$ (the case $n=1$ is trivial), the middle term is a free $k[\mathbf{y}]$-module and the last term is a free $k\left[y_{1}, \ldots, y_{n-1}\right]$-module. All terms are graded $k[\mathbf{y}]$-modules, finitely generated in each $x$-degree (see 44.5 for more explanation). It follows that the first term is a free $k[\mathbf{y}]$-module.

4.3. Overview. Before embarking on the proof of Theorem4.1, let us outline our method. We will actually prove a stronger result, by induction on $n$ and $l$, namely, 
that $R=R(n, l)$ is free with a basis $B$ which is a common basis for a certain family of ideals in $R$. By this we mean that each ideal in the family is itself a free module, spanned by a subset of the overall basis $B$. We remark that this is only possible because the family of ideals we consider is rather special: inside the lattice of all ideals of $R(n, l)$, with intersection and sum as lattice operations, our family generates a distributive sublattice $\mathcal{L}$, all of whose members are radical ideals, and such that $R(n, l) / I$ is a free $k[\mathbf{y}]$-module for all $I \in \mathcal{L}$.

The ideals $I$ in our family will be the ideals of certain subspace arrangements contained as closed subsets within the polygraph $Z(n, l)$. Before proceeding further, we define these new subspace arrangements, and state the theorem to be proven in its full strength.

Definition 4.3.1. Let $Z(n, l)$ be a polygraph. Given integers $r \in[n] \cup\{0\}, k \in$ $[l] \cup\{0\}$ and $m$, we denote by $Y(m, r, k)$ the subspace arrangement

$$
Y(m, r, k)=\bigcup_{f, T} V\left(x_{j}: j \in T\right) \cap W_{f},
$$

where $f$ ranges over functions $f:[l] \rightarrow[n]$, as in Definition 4.1.1, and $T$ ranges over subsets of $[n]$ such that

$$
|T \cap[r] \backslash f([k])| \geq m
$$

We denote by $I(m, r, k)$ the ideal of $Y(m, r, k)$ as a reduced closed subscheme of $Z(n, l)$.

Roughly speaking, $Y(m, r, k)$ consists of points at which $x_{j}$ vanishes for at least $m$ indices $j \in[r]$ such that $j \neq f(i)$ for $i \leq k$. Stated this way, the criterion for membership in $Y(m, r, k)$ is ambiguous for points lying on more than one $W_{f}$. A precise formulation will be given in $\$ 4.7$.

Note that in some cases $Y(m, r, k)$ is trivial - either empty or equal to $Z(n, l)$. Specifically, if $m \leq 0$, then $Y(m, r, k)$ is the whole of $Z(n, l)$ and $I(m, r, k)=0$. If $m>r$, or if $m=r=n$ and $k>0$, then $Y(m, r, k)$ is empty and $I(m, r, k)=(1)$. Of course we could have simply ruled out the trivial cases by definition, but it will simplify notation later on to admit them.

Now we state the full theorem to be proven by induction.

Theorem 4.2. The coordinate ring $R(n, l)$ of the polygraph $Z(n, l)$ is a free $k[\mathbf{y}]$ module with a basis $B$ such that every ideal $I(m, r, k)$ is spanned as a $k[\mathbf{y}]$-module by a subset of $B$.

The proof of Theorem 4.2 will occupy us for most of the rest of this section. Our strategy is to construct the common ideal basis $B$ by induction, using bases that we may assume are already given in $R(n-1, l)$ and $R(n, l-1)$. The induction proceeds in three stages. First we construct a basis of $R(n, l) / I(1,1, l)$ from a basis of $R(n-1, l)$, then we construct a basis of $R(n, l) / I(1,1, t-1)$ from a basis of $R(n, l) / I(1,1, t)$, for $t$ descending from $l$ to 1 , and finally we construct a basis of $R(n, l)$ from a basis of $R(n, l) / I(1,1,0)$.

In order to show that the sets we construct are bases, we rely on a simple but crucial algebraic device. Because we only consider arrangements of subspaces on which the $\mathbf{y}$ coordinates are independent, their coordinate rings are torsionfree $k[\mathbf{y}]$-modules. To prove that a subset of a torsion-free $k[\mathbf{y}]$-module is a free module basis, it suffices to verify it locally on an open locus $\hat{U}_{2} \subseteq \operatorname{Spec} k[\mathbf{y}]$ whose 
complement has codimension two. In our situation there is a natural choice of the open set $\hat{U}_{2}$, namely, the set of points where at most two of the $\mathbf{y}$ coordinates coincide. We shall see that the local geometry of $Z(n, l)$ over $\hat{U}_{2}$ essentially reduces to the case $n=2$. In this case we are able to verify everything explicitly.

Several sources of difficulty make the proof more complicated than the program just outlined would suggest. The first difficulty is that we sometimes have to use facts we can only verify over $\hat{U}_{2}$ not just to prove that our elements form a basis, but to construct them in the first place. This forces us to employ a delicate method in the second and third stages of the basis construction process. From an inductively constructed common ideal basis, we first produce a less restrictive kind of basis. We then define elements of our new common ideal basis in terms of the less restrictive basis, allowing the coefficients to be rational functions of $\mathbf{y}$. Finally we use information from the case $n=2$ to show that the coefficients are regular on $\hat{U}_{2}$, and hence they are polynomials.

Even in the construction of the less restrictive basis, the role of the inductively constructed common ideal basis is quite subtle. Our ability to make use of it depends on fortunate scheme-theoretic relationships between the subspace arrangements $Y(m, r, k)$ and certain other special arrangements. Most of the work in $\S \$ 4.9$ and 4.10 goes into establishing and carefully exploiting these relationships.

The easier, preliminary portions of the proof are in $\$ \$ 4.44 .8$. Some of this preliminary material is conceptually fundamental, especially the local reduction in 84.4 the description of the basis and the ideals $I(m, r, k)$ in the case $n=2$ in \$4.6. and the general basis lifting method in $\$ 4.8$. The preliminary material also includes various lemmas giving assorted details about the $n=2$ picture and the corresponding local picture over $\hat{U}_{2}$. The reader might do well to skip the proofs of these lemmas at first, returning to them after seeing how they are used later on. As a guide to the reader we now summarize the contents of the remaining subsections.

\$4.4 Definition of the open sets $U_{1}$ and $U_{2}$ and reduction of the local geometry of $Z(n, l)$ to the case $n=2$.

$\$ 4.5$ Double grading of $R(n, l)$ and computation of $x$-degree Hilbert series for generic $\mathbf{y}$.

\$4.6 Full working out of the case $n=2$ and some of its consequences.

\$4.7 Further information about the arrangements $Y(m, r, k)$.

$\$ 4.8$ General lifting principle for extending common ideal bases to schemes with an extra coordinate.

$\$ 4.9$ Application of the lifting principle in the case of special arrangements.

$\$ 4.10$ The three stages of the basis construction procedure.

$\$ 4.11$ Proofs of Theorems 4.1 and 4.2 and Proposition 3.8.1.

$\$ 4.12$ Extension of Theorem 4.1 to arbitrary ground rings.

The geometric results in $\$ 3$ only depend on the contents of $\$ 4.1$ and $\S \$ 4.34 .11$ The material in 4.2 and 4.12 has been included for the sake of illustration and completeness.

We close this subsection with a brief discussion of the base cases for the induction. Theorem 4.2 is essentially trivial for $n=1$. This case can therefore serve as the base of induction on $n$, although we still need to work out the case $n=2$ in full detail since it is used in the induction step for $n>1$. The base of induction on $l$ will be the case $l=0$. This case is not altogether trivial, and already usefully 
illustrates both Theorem 4.2 and the definition of the arrangements $Y(m, r, k)$. For this reason we give it here rather than later.

Lemma 4.3.2. Theorem 4.2 holds for $Z(n, 0)$. More precisely, we have $R(n, 0)=$ $k[\mathbf{x}, \mathbf{y}]$, and the set of all monomials in the variables $\mathbf{x}$ is a basis of the required type.

Proof. We have $Z(n, 0)=E^{n}$ and hence $R(n, 0)=k[\mathbf{x}, \mathbf{y}]$. Obviously $R(n, 0)$ is a free $k[\mathbf{y}]$-module, but there are also the ideals $I(m, r, k)$ to consider. Necessarily we must have $k=0$. Then $Y(m, r, 0)$ is the union of subspaces in $E^{n}$ defined by the vanishing of at least $m$ of the coordinates $x_{1}, \ldots, x_{r}$. Its ideal $I(m, r, 0)$ is generated by all square-free monomials $\prod_{j \in T} x_{j}$, where $T$ is a subset of $[r]$ of size $|T|=r-m+1$. The set $B$ of all monomials in the $\mathbf{x}$ coordinates is a free $k[\mathbf{y}]$ module basis of $R(n, 0)$, with subsets spanning every ideal generated by monomials in $\mathbf{x}$. In particular, each ideal $I(m, r, 0)$ is spanned by a subset of $B$.

4.4. Local geometry of $Z(n, l)$. Nearly everything in our proof of Theorem 4.2 ultimately depends on a process of local geometric reduction over certain open sets $\hat{U}_{1}$ and $\hat{U}_{2}$ to the cases $n=1$ (which is trivial) and $n=2$ (which we will examine in detail). Here we define the relevant open sets, and set up the required algebraic, geometric and notational machinery.

Definition 4.4.1. The set $\hat{U}_{k}$ is the open locus in Spec $k[\mathbf{y}]$ where the coordinates $y_{1}, \ldots, y_{n}$ assume at least $n+1-k$ distinct values. In other words, $\hat{U}_{k}$ is the complement of the union of all linear subspaces $V=V\left(y_{i_{1}}-y_{j_{1}}, \ldots, y_{i_{k}}-y_{j_{k}}\right)$ defined by $k$ independent forms $\left(y_{i}-y_{j}\right)$. In particular, $\hat{U}_{1}$ is the locus where the $y_{i}$ are all distinct, and $\hat{U}_{2}$ is the locus where there is at most one coincidence $y_{p}=y_{q}$. For any scheme $\pi: Z \rightarrow \operatorname{Spec} k[\mathbf{y}]$ over Spec $k[\mathbf{y}]$, we define $U_{k} \subseteq Z$ to be the open set $\pi^{-1}\left(\hat{U}_{k}\right)$.

The definition of $U_{k}$ involves an abuse of notation, since we might, for instance, have $U_{k}$ defined as a subset of $E^{n} \times E^{l}$ in one place and as a subset of $Z(n, l)$ in another. In practice it will be clear from the context what is meant. Note that the definitions are consistent in the sense that the subset $U_{k} \subseteq Z(n, l)$ is the intersection of $Z(n, l)$ with the subset $U_{k} \subseteq E^{n} \times E^{l}$.

To study $R(n, l)$ as a $k[\mathbf{y}]$-module, we will want to localize with respect to prime ideals in $k[\mathbf{y}]$, that is, at points $Q \in \hat{U}_{k} \subseteq \operatorname{Spec} k[\mathbf{y}]$. To extract local geometric information about $Z(n, l)$ as a subscheme of $E^{n} \times E^{l}$, by contrast, we want to localize at points $P \in U_{k} \subseteq E^{n} \times E^{l}$. The following lemma relates these two types of localization.

Lemma 4.4.2. Let $R$ be a $k[\mathbf{y}]$-algebra, let $\pi: \operatorname{Spec} R \rightarrow \operatorname{Spec} k[\mathbf{y}]$ be the projection on the $\mathbf{y}$ coordinates, let $\hat{U}$ be an open subset of Spec $k[\mathbf{y}]$, and let $U=\pi^{-1}(\hat{U})$, as in Definition 4.4.1. If $I, J \subseteq R$ are ideals such that $I_{P}=J_{P}$ locally for all $P \in U$ (localized as $R$-modules), then $I_{Q}=J_{Q}$ for all $Q \in \hat{U}$ (localized as $k[\mathbf{y}]$ modules).

Proof. The points of Spec $R_{Q}$ are exactly the ideals $P_{Q}$, where $P \in \operatorname{Spec} R$ is such that $\pi(P) \subseteq Q$. In particular, every such $P$ belongs to $U$, for $Q \in \hat{U}$. Since $\left(I_{Q}\right)_{P_{Q}}=I_{P}=J_{P}=\left(J_{Q}\right)_{P_{Q}}$ for all such $P$, we have $I_{Q}=J_{Q}$.

We will really only be interested in the open sets $U_{1}$ and $U_{2}$, where we can fully understand the local geometry of $Z(n, l)$. Since $Z(n, l)$ and every other subspace 
arrangement we consider consists of subspaces on which the $\mathbf{y}$ coordinates are independent, $U_{1}$ and $U_{2}$ are dense and have complements of codimension 1 and 2 respectively. As we shall see, it is possible to extract significant global geometric information from the careful use of local information on $U_{1}$ or $U_{2}$. The following lemma shows that the local geometry of $Z(n, l)$ on $U_{1}$ is essentially trivial.

Lemma 4.4.3. The components $W_{f}$ of $Z(n, l)$ have disjoint intersections with $U_{1}$. Thus for $P \in U_{1} \cap Z(n, l)$, there is a unique $W_{f}$ containing $P$, and $Z(n, l)$ coincides locally with $W_{f}$, that is, $I(Z(n, l))_{P}=\left(I_{f}\right)_{P}$.

Proof. Let $P$ be a point of $W_{f} \cap W_{g}$, where $f(i) \neq g(i)$ for some $i$. On $W_{f}$ we have $b_{i}=y_{f(i)}$, while on $W_{g}$ we have $b_{i}=y_{g(i)}$. Hence $P \in V\left(y_{f(i)}-y_{g(i)}\right)$, so $P \notin U_{1}$.

Here is one easy but useful consequence of the local picture on $U_{1}$. Recall that the lattice of ideals in a ring $R$ is the set of all ideals in $R$, with meet and join operations given by intersection and sum.

Lemma 4.4.4. Let $\mathcal{L}$ be the sublattice of the lattice of ideals in $R(n, l)$ generated by the ideals of all subspaces of the form

$$
V\left(x_{j}: j \in T\right) \cap W_{f} .
$$

Then for every $I \in \mathcal{L}, V(I) \cap U_{1}$ is reduced, that is, $I_{P}=\sqrt{ } I_{P}$ for all $P \in U_{1}$.

Proof. At a point $P \in U_{1}, Z(n, l)$ coincides locally with $W_{g} \cong E^{n}$ for a unique $g$. Identifying $\mathcal{O}\left(W_{g}\right)$ with $\mathcal{O}\left(E^{n}\right)=k[\mathbf{x}, \mathbf{y}]$, the ideal of the subspace in (75) is locally either $\left(x_{j}: j \in T\right)$, if $g=f$, or $(1)$, otherwise. It follows that every $I \in \mathcal{L}$ coincides locally with an ideal in $k[\mathbf{x}, \mathbf{y}]$ generated by square-free monomials in the variables $\mathbf{x}$, and for such an ideal we have $I=\sqrt{ } I$.

Corollary 4.4.5. If $I$ belongs to the lattice generated by the ideals $I(m, r, k)$ in $R(n, l)$, then $V(I) \cap U_{1}$ is reduced.

Next we want to give the analog of Lemma 4.4.3 for $U_{2}$, showing that the local geometry of $Z(n, l)$ at a point of $U_{2}$ essentially reduces to the case $n=2$. To state our next lemma precisely, we will need to consider the following type of situation. Let $Z \subseteq Z(n, l)$ be the union of those components $W_{f}$ of $Z(n, l)$ for which $f(i)$ takes some preassigned value $h(i)$, for all $i$ in a subset $I$ of the index set $[l]$. On $Z$ we have identically $a_{i}=x_{h(i)}, b_{i}=y_{h(i)}$ for $i \in I$. Using these equations to eliminate the coordinates $a_{i}, b_{i}$ for $i \in I$ we see that $Z$ is isomorphic to a polygraph $Z(n, l-j) \subseteq E^{n} \times E^{l-j}$, where $j=|I|$.

This situation creates a problem of notation, as the natural index set for the coordinates on $E^{l-j}$ here is not $[l-j]$ but $L=[l] \backslash I$. Similar problems arise involving the index set $[n]$, for example if $Z$ is the union of those components $W_{f}$ for which $f$ takes values in a subset $N \subseteq[n]$. As such situations will arise repeatedly in what follows, we adopt the following notational convention to deal with them.

Convention 4.4.6. Let $N$ and $L$ be finite sets of positive integers of sizes $|N|=n$, $|L|=l$. To every construct that we will define in terms of $n$ and $l$, there is a corresponding construct in which the roles of $[n]$ and $[l]$ are played by $N$ and $L$, respectively. We will refer to the $N, L$ version as the construct in indices $N, L$. 
A few examples should suffice to make the meaning of this convention clear. In indices $N, L$, the ambient space $E^{n} \times E^{l}$ is replaced by the space $E^{N} \times E^{L}$ with coordinates

$$
\mathbf{x}_{N}, \mathbf{y}_{N}, \mathbf{a}_{L}, \mathbf{b}_{L}=x_{j_{1}}, y_{j_{1}}, \ldots, x_{j_{n}}, y_{j_{n}}, a_{i_{1}}, b_{i_{1}}, \ldots, a_{i_{l}}, b_{i_{l}},
$$

where $N=\left\{j_{1}, \ldots, j_{n}\right\}$ and $L=\left\{i_{1}, \ldots, i_{l}\right\}$. Functions $f:[l] \rightarrow[n]$ become functions $f: L \rightarrow N$, the subspaces $W_{f}$ in indices $N, L$ have the obvious meaning, and their union is the polygraph $Z(N, L)$ in indices $N, L$. Its coordinate ring is $R(N, L)$. The arrangements $Y_{N, L}(m, r, k)$ and their ideals $I_{N, L}(m, r, k)$ are defined as in 4.3.1 but with $[r]$ and $[k]$ referring to the smallest $r$ elements of $N$ and the smallest $k$ elements of $L$, respectively.

Using the above convention we can readily describe the local geometry of $Z(n, l)$ at points of $U_{2}$.

Lemma 4.4.7. Let $P$ be a point of $U_{2} \backslash U_{1}$ and let $\{p, q\}$ be the unique pair of indices such that $P \in V\left(y_{p}-y_{q}\right)$. Let $\sim$ be the equivalence relation on functions $f:[l] \rightarrow[n]$ defined by $f \sim g$ if and only if for all $i \in[l], y_{f(i)}-y_{g(i)}$ vanishes at $P$, that is, $f(i)=g(i)$ or $\{f(i), g(i)\}=\{p, q\}$.

(i) We have $P \in W_{f}$ only for $f$ in a unique $\sim$-equivalence class $F$, so $Z(n, l)$ coincides locally at $P$ with

$$
Z=\bigcup_{f \in F} W_{f}
$$

(ii) Let $h$ be a member of $F$, let $N=\{p, q\}$ and let $L=h^{-1}(N)$ (note that $L$ depends only on $F)$. The projection of $Z$ on the coordinates $\mathbf{x}, \mathbf{y}, \mathbf{a}_{L}, \mathbf{b}_{L}$ is an isomorphism

$$
Z \cong E^{[n] \backslash N} \times Z(N, L),
$$

where $Z(N, L)$ is the polygraph in indices $N$ and $L$.

Proof. If $P \in W_{f} \cap W_{g}$, then for all $i$ we have $P \in V\left(y_{f(i)}-y_{g(i)}\right)$, just as in the proof of Lemma 4.4.3. This implies (i). On $Z$ we have identically $a_{i}=x_{h(i)}, b_{i}=y_{h(i)}$ for $i \notin L$, so the coordinate ring of $Z$ is generated by the remaining variables, namely $\mathbf{x}, \mathbf{y}, \mathbf{a}_{L}, \mathbf{b}_{L}$. This implies that the projection on these coordinates is an isomorphism of $Z$ onto its image, which is clearly $E^{[n] \backslash N} \times Z(N, L)$.

Reasoning as in the proof of part (ii) of the preceding lemma, we also obtain the following ideal-theoretic result, which we record for future reference.

Lemma 4.4.8. Let $I \subseteq[l]$ and $h: I \rightarrow[n]$ be given, and set

$$
Z=\bigcup W_{f}, \quad \text { over } f:[l] \rightarrow[n] \text { such that }\left.f\right|_{I}=h,
$$

a subarrangement of $Z(n, l)$. Setting $L=[l] \backslash I$, the projection of $Z$ on the coordinates $\mathbf{x}, \mathbf{y}, \mathbf{a}_{L}, \mathbf{b}_{L}$ is an isomorphism $Z \cong Z([n], L)$, and the ideal of $Z$ as a closed subscheme of $Z(n, l)$ is given by

$$
I(Z)=\sum_{i \in I}\left(a_{i}-x_{h(i)}, b_{i}-y_{h(i)}\right)
$$

Proof. Let $\pi$ be the projection on the coordinates $\mathbf{x}, \mathbf{y}, \mathbf{a}_{L}, \mathbf{b}_{L}$. On $Z$ we have identically $a_{i}=x_{h(i)}, b_{i}=y_{h(i)}$ for $i \notin L$. As in the proof of the preceding lemma, this implies that $\pi$ induces an isomorphism of $Z$ onto its image, which in this case is $Z([n], L)$. Note that $Z([n], L)$ is also the image under $\pi$ of the whole of $Z(n, l)$. 
Let $J$ be the ideal on the right-hand side in (178), and let $\tilde{Z}=V(J)$. Then $\tilde{Z}$ is a closed subscheme of $Z(n, l)$, conceivably nonreduced. Modulo $J$ we again have $a_{i}=x_{h(i)}, b_{i}=y_{h(i)}$ for $i \notin L$, so $\pi$ induces an isomorphism of $\tilde{Z}$ onto its (scheme-theoretic) image. But we have $Z \subseteq \tilde{Z} \subseteq Z(n, l)$, and $Z([n], L)$ is the image of both $Z$ and $Z(n, l)$. Since $Z$ and $Z(n, l)$ are reduced, this holds scheme-theoretically as well as set-theoretically. It follows that $Z([n], L)$ is also the scheme-theoretic image $\pi(\tilde{Z})$. The isomorphism $\pi: Z \cong Z([n], L)$ factors as the isomorphism $\pi: \tilde{Z} \cong Z([n], L)$ composed with the closed embedding $Z \hookrightarrow \tilde{Z}$, so this implies $Z=\tilde{Z}$ or equivalently $I(Z)=J$.

We come now to a technical lemma which despite its simplicity is really the motor of the whole machine. It allows us to verify that a purported free $k[\mathbf{y}]$-module basis (of $R(n, l)$, for instance) really is one by restricting our attention to $\hat{U}_{2}$, where we have good control of the local geometry.

Lemma 4.4.9. Let $M$ be a torsion-free $k[\mathbf{y}]$-module, and let $B$ be a subset of $M$. Suppose that for every $Q \in \hat{U}_{2}$, the localization $M_{Q}$ is a free $k[\mathbf{y}]_{Q}$-module with basis $B$. Then $M$ is a free $k[\mathbf{y}]$-module with basis $B$.

Proof. We are to show that every $x \in M$ can be uniquely expressed as

$$
x=\sum_{\alpha} p_{\alpha} b_{\alpha}
$$

with $p_{\alpha} \in k[\mathbf{y}]$ and $b_{\alpha} \in B$. By hypothesis, this is true for the image of $x$ in $M_{Q}$, with $p_{\alpha} \in k[\mathbf{y}]_{Q}$, for all $Q \in \hat{U}_{2}$. The local ring $k[\mathbf{y}]_{Q}$ is a subring of $k[\mathbf{y}]_{(0)}=k(\mathbf{y})$, and the unique coefficients $p_{\alpha} \in k[\mathbf{y}]_{Q} \subseteq k(\mathbf{y})$ satisfying (79) for any $Q$ also satisfy (79) for $Q=0$. Hence they do not depend on $Q$. Since the complement of $\hat{U}_{2}$ has codimension 2, every rational function regular on $\hat{U}_{2}$ is regular everywhere. Thus the $p_{\alpha}$ belong to $k[\mathbf{y}]$. Since $M$ is torsion-free and (179) holds locally on the dense open set $\hat{U}_{2}$, (79) holds identically.

Corollary 4.4.10. Let $I$ and $J$ be free submodules of a torsion-free $k[\mathbf{y}]$-module $M$ and suppose that $I_{Q}=J_{Q}$ for all $Q \in \hat{U}_{2}$. Then $I=J$.

Proof. Lemma 4.4 .9 implies that any free $k[\mathbf{y}]$-module basis of $I$ is also a basis of $I+J$, so $I=I+J$. Similarly $J=I+J$.

We should stress that the role of the explicit basis $B$ in Lemma 4.4.9 is crucial. In general, a torsion-free $k[\mathbf{y}]$-module $M$ which is locally free on $\hat{U}_{2}$ certainly need not be free. As an example, take $M$ to be an ideal in $k[\mathbf{y}]$ with $V(M)$ nonempty and disjoint from $\hat{U}_{2}$. Then $M_{Q}=(1)_{Q}$ for all $Q \in \hat{U}_{2}$, so $M$ is locally free with basis $\{1\}$ on $\hat{U}_{2}$. But no element contained in $M$ generates $M$ locally on $\hat{U}_{2}$, so we cannot conclude that $M$ is free. Indeed if $M$ were free, Corollary 4.4.10 would then imply $M=(1)$.

The following companion to Lemma 4.4.9 is useful to establish that a known basis of a free $k[\mathbf{y}]$-module (of $R(n, l)$, for instance) is a common basis for a submodule or submodules (such as the ideals $I(m, r, k)$ ).

Lemma 4.4.11. Let $B$ be a basis of a free $k[\mathbf{y}]$-module $M$. Let $J$ be a submodule of $M$, and suppose that $B_{1}=B \cap J$ spans $k(\mathbf{y}) \otimes J$. Then $J=k[\mathbf{y}] B_{1}$. 
Proof. Let $x$ be an element of $J$. Since $M$ is free with basis $B$ we can write

$$
x=\sum_{\alpha} p_{\alpha} b_{\alpha}
$$

with $p_{\alpha} \in k[\mathbf{y}]$ and $b_{\alpha} \in B$. Of course this is also the unique expression for $x$ in terms of the basis $B$ of the $k(\mathbf{y})$-vector space $k(\mathbf{y}) \otimes M$. Hence $p_{\alpha}=0$ for $b_{\alpha} \notin B_{1}$, so we have $x \in k[\mathbf{y}] B_{1}$.

Note that the condition that $B_{1}$ spans $k(\mathbf{y}) \otimes J$ can be checked locally on $\hat{U}_{1}$, since tensoring with $k(\mathbf{y})$ is the same as localizing at $Q=0$, and the zero ideal belongs to every nonempty open subset of Spec $k[\mathbf{y}]$. The philosophy governing the application of Lemmas 4.4.9 and 4.4.11 is therefore as follows. To show that a candidate $B$ is a free module basis, we can check it locally on $\hat{U}_{2}$; then to show that $B$ is a common ideal basis, we can check it locally on $\hat{U}_{1}$.

4.5. Generic Hilbert series. Our next task is to work out the case $n=2$ in detail. This will be done in \$4.6, using what amounts to a Gröbner basis argument in disguise. To make this argument work, we need some enumerative information in advance about the Hilbert series of $R(n, l)$. Here we gather the required information. We also note the general fact that $R(n, l)$ is doubly graded and finite over $k[\mathbf{x}, \mathbf{y}]$, a fact that will later be used implicitly in several places.

The coordinate ring

$$
\mathcal{O}\left(E^{n} \times E^{l}\right)=k[\mathbf{x}, \mathbf{y}, \mathbf{a}, \mathbf{b}]
$$

of $E^{n} \times E^{l}$ is doubly graded, by degree in the $\mathbf{x}$, a variables (or $x$-degree) and the $\mathbf{y}, \mathbf{b}$ variables $\left(y\right.$-degree) respectively. The ideals $I_{f}$ are obviously doubly homogeneous, and since the defining ideal of $Z(n, l)$ is their intersection, the coordinate ring

$$
R(n, l)=k[\mathbf{x}, \mathbf{y}, \mathbf{a}, \mathbf{b}] / I(Z(n, l))
$$

is doubly graded. All ideals considered throughout will be doubly homogeneous, and all coordinate rings doubly graded.

By construction $Z(n, l)$ is finite over $E^{n}$, so $R(n, l)$ is a finitely generated $k[\mathbf{x}, \mathbf{y}]$ module. Hence if

$$
R(n, l)=\bigoplus_{d} R(n, l)_{d}
$$

is the grading of $R(n, l)$ by $x$-degree, then each homogeneous component $R(n, l)_{d}$ is a finitely generated graded $k[\mathbf{y}]$-module (graded by $y$-degree). We have the following well-known graded version of Nakayama's lemma.

Lemma 4.5.1. Let $M$ be a finitely generated graded $k[\mathbf{y}]$-module. If $B$ is a set of homogeneous elements of $M$ that spans $M / \mathbf{y} M$ as a $k$-vector space, then $B$ generates $M$. If $B$ further satisfies $|B|=\operatorname{dim}_{k(\mathbf{y})}(k(\mathbf{y}) \otimes M)$, then $M$ is a free $k[\mathbf{y}]$-module with basis $B$.

For the coordinate ring $R$ of any union of subspaces of the form $V\left(x_{j}: j \in\right.$ $T) \cap W_{f}$, including $Z(n, l)$ and $Y(m, r, k)$, we can readily determine the Hilbert series of $k(\mathbf{y}) \otimes R$ as a graded $k(\mathbf{y})$-algebra (graded by $x$-degree). 
Lemma 4.5.2. Let $R$ be the coordinate ring of a union of subspaces

$$
\bigcup_{C} V\left(x_{j}: j \in T\right) \cap W_{f},
$$

over some collection $C$ of pairs $T \subseteq[n], f:[l] \rightarrow[n]$. Then $R$ is torsion-free as a $k[\mathbf{y}]$-module, and the dimension of the $x$-degree homogeneous component $k(\mathbf{y}) \otimes R_{d}$ is equal to the number of pairs

$$
e \in \mathbb{N}^{n}, \quad f:[l] \rightarrow[n]
$$

such that $e_{1}+\cdots+e_{n}=d$ and there is some $(T, f) \in C$ for this $f$ with $e_{j}=0$ for all $j \in T$.

Proof. Let $W_{T, f}$ denote the subspace $V\left(x_{j}: j \in T\right) \cap W_{f}$, and let $I_{T, f}=I_{f}+\left(x_{j}\right.$ : $j \in T)$ be its ideal and $R_{T, f}=k[\mathbf{x}, \mathbf{y}, \mathbf{a}, \mathbf{b}] / I_{T, f}$ its coordinate ring. By definition, $R=k[\mathbf{x}, \mathbf{y}, \mathbf{a}, \mathbf{b}] / I$, where $I$ is the intersection of the ideals $I_{T, f}$ for all $(T, f) \in C$. Since the $\mathbf{y}$ coordinates are independent on each $W_{T, f}$, the coordinate rings $R_{T, f}$ are free, and hence torsion-free, $k[\mathbf{y}]$-modules. The ring $R$ is isomorphic to a subring of the direct sum $\bigoplus_{C} R_{T, f}$, so $R$ is also a torsion-free $k[\mathbf{y}]$-module.

Let $C_{f}$ be the set of pairs $(T, f) \in C$ with a given $f$, and let $R_{f}$ be the coordinate ring of the partial union $Z_{f}=\bigcup_{C_{f}} W_{T, f}$. We have an injective ring homomorphism

$$
R \hookrightarrow \bigoplus_{f} R_{f}
$$

By Lemma 4.4.3 the partial unions $Z_{f}$ have disjoint restrictions to $U_{1}$. By Lemma 4.4.2 this implies that (86) localizes to an isomorphism at each point of $\hat{U}_{1}$ and, in particular, upon tensoring with $k(\mathbf{y})$.

Now the projection of $W_{f}$ on $E^{n}$ is an isomorphism, so $Z_{f}$ projects isomorphically on $V \times \operatorname{Spec} k[\mathbf{y}]$, where $V$ is the union of coordinate subspaces $\bigcup_{C_{f}} V\left(x_{j}: j \in T\right)$ in Spec $k[\mathbf{x}]$. The coordinate ring of $V$, say $k[\mathbf{x}] / J$, is the face ring of a simplicial complex. It has a homogeneous vector space basis consisting of all monomials $\mathbf{x}^{e}$ such that there is some $(T, f) \in C_{f}$ with $e_{j}=0$ for all $j \in T$. The ring $R_{f}$ in turn is a free $k[\mathbf{y}]$-module with this same basis. Since $k(\mathbf{y}) \otimes R \cong \bigoplus_{f} k(\mathbf{y}) \otimes R_{f}$, the result follows.

Corollary 4.5.3. The Hilbert series of $k(\mathbf{y}) \otimes R(n, l)$ as a $k(\mathbf{y})$-algebra graded by $x$-degree is given by

$$
\sum_{d} t^{d} \operatorname{dim}_{k(\mathbf{y})}\left(k(\mathbf{y}) \otimes R(n, l)_{d}\right)=\frac{n^{l}}{(1-t)^{n}} .
$$

In 4.6 we will make use of Lemma 4.5.1 and Corollary 4.5.3 in the following guise.

Corollary 4.5.4. Let $B$ be a set of doubly homogeneous polynomials whose images in $R(n, l)$ span $R(n, l) /(\mathbf{y})$ as a $k$-vector space. Denoting the $x$-degree of $p \in B$ by $d(p)$, suppose the degree enumerator of $B$ satisfies

$$
\sum_{p \in B} t^{d(p)}=\frac{n^{l}}{(1-t)^{n}} .
$$

Then $R(n, l)$ is a free $k[\mathbf{y}]$-module with basis $B$. 
We also have a version of Corollary 4.5 .3 for the arrangements $Y(m, r, k)$.

Corollary 4.5.5. The Hilbert series

$$
\sum_{d} t^{d} \operatorname{dim}_{k(\mathbf{y})}\left(k(\mathbf{y}) \otimes \mathcal{O}(Y(m, r, k))_{d}\right)
$$

of $k(\mathbf{y}) \otimes \mathcal{O}(Y(m, r, k))$ as a graded $k(\mathbf{y})$-algebra is equal to the enumerator

$$
\sum_{e, f} t^{|e|}, \quad \text { over } e \in \mathbb{N}^{n}, f:[l] \rightarrow[n] \text { satisfying }\left|[r] \backslash S_{k}(e, f)\right| \geq m,
$$

where $|e|=e_{1}+\cdots+e_{n}$ and $S_{k}(e, f)=\left\{j: e_{j}>0\right\} \cup f([k])$.

Proof. The requirement that $e_{j}=0$ for all $j \in T$, for a given $f$ and some $T$ satisfying (74), is equivalent to $\left|[r] \backslash S_{k}(e, f)\right| \geq m$.

4.6. The case $n=2$. At this point we are ready to analyze the case $n=2$ in full detail. Apart from our later need for the results, we hope the reader may find that working out the case $n=2$ usefully illustrates the concepts introduced so far. We begin by writing down explicit polynomials that will form the common ideal basis required by the conclusion of Theorem 4.2 For this discussion we fix $l$, and of course we fix $n=2$.

To each pair $(e, f)$, for $e \in \mathbb{N}^{2}$ and $f:[l] \rightarrow[2]$, we will associate a basis element $p[e, f]$, homogeneous of $x$-degree $|e|=e_{1}+e_{2}$.

For $e=(0,0)$ we set

$$
p[e, f]=\prod_{\substack{j>1 \\ f(j) \neq f(1)}}\left(b_{j}-b_{1}\right) \cdot \begin{cases}\left(b_{1}-y_{2}\right) & \text { if } f(1)=1, \\ 1 & \text { otherwise. }\end{cases}
$$

For $e=(0, h)$ with $h>0$, let $f^{-1}(\{1\})=S \cup T$, where $S$ and $T$ are disjoint and $S$ consists of the smallest $h$ elements of $f^{-1}(\{1\})$, or the whole set if $h \geq\left|f^{-1}(\{1\})\right|$. Then we set

$$
p[e, f]=x_{2}^{h-|S|} \prod_{i \in S}\left(a_{i}-x_{1}-x_{2}\right) \prod_{j \in T}\left(b_{j}-y_{2}\right) .
$$

For $e=(h, 0)$ with $h>0$ we set

$$
p[e, f]=x_{1} \theta p[(0, h-1), \theta f],
$$

where $\theta$ denotes the transposition (12), acting on $f$ in the obvious way, and on the polynomial ring $k[\mathbf{x}, \mathbf{y}, \mathbf{a}, \mathbf{b}]$ by exchanging $x_{1}$ with $x_{2}$ and $y_{1}$ with $y_{2}$, while fixing the coordinates $\mathbf{a}, \mathbf{b}$.

Finally, for $e=\left(h_{1}, h_{2}\right)$ with both $h_{1}, h_{2}>0$ we set $h=\min \left(h_{1}, h_{2}\right)$ and

$$
p[e, f]=\left(x_{1} x_{2}\right)^{h} p[e-(h, h), f] .
$$

The complicated definition of the elements $p[e, f]$ is forced on us by the requirement that they should form a common basis for the ideals $I(m, r, k)$, with the rule for membership in $I(m, r, k)$ being given by Lemma 4.6.2, below. To help orient the reader let us consider the simplest example. For $e=(0,0)$ and $f$ equal to the constant function $f(i)=2$, we have $p[e, f]=1$. For these $e, f$, the rule in Lemma 4.6.2 places $p[e, f]$ only in the ideals $I(m, r, k)$ with $m>r$ and $I(2,2, k)$ with $k>0$, which are trivially equal to (1). 
Lemma 4.6.1. For $n=2$, the coordinate ring $R(2, l)$ of $Z(2, l)$ is a free $k[\mathbf{y}]$ module with basis $B$ the set of elements $p[e, f]$ defined in (91)-(94).

Proof. We will show that $B$ spans $R(2, l) /(\mathbf{y})$ as a $k$-vector space. Since the $x$ degree enumerator of $B$ is clearly $2^{l} /(1-t)^{2}$, this implies the result by Corollary 4.5.4.

Let $B_{0}=\{p[(0, h), f]\}$ be the subset of $B$ containing only the elements defined in (91) and (92). By (93) and (94) we have

$$
B=\left(B_{0} \cup x_{1} \theta B_{0}\right) \cdot\left\{1, x_{1} x_{2},\left(x_{1} x_{2}\right)^{2}, \ldots\right\} .
$$

It suffices to show that $B_{0} \cup x_{1} \theta B_{0}$ spans $S=R(2, l) /\left(\left(x_{1} x_{2}\right)+(\mathbf{y})\right)$. For this it suffices in turn to show that $B_{0}$ spans $S /\left(x_{1}\right)$ and $x_{1} \theta B_{0}$ spans $x_{1} S$. Since $x_{1} x_{2}=0$ in $S$, multiplication by $x_{1}$ gives a well-defined surjective homomorphism $S /\left(x_{2}\right) \rightarrow x_{1} S$. If $B_{0}$ spans $S /\left(x_{1}\right)$, then $\theta B_{0}$ spans $S /\left(x_{2}\right)$, and therefore $x_{1} \theta B_{0}$ spans $x_{1} S$. Hence we need only show that $B_{0}$ spans $S /\left(x_{1}\right)$.

The ideal of $Z(2, l)$ contains the ideal

$$
\sum_{i \in[l]}\left(a_{i}-x_{1}, b_{i}-y_{1}\right)\left(a_{i}-x_{2}, b_{i}-y_{2}\right)+\sum_{i, j \in[l]}\left(\operatorname{det}\left[\begin{array}{lll}
a_{i} & b_{i} & 1 \\
a_{j} & b_{j} & 1 \\
x_{1} & y_{1} & 1
\end{array}\right]\right) .
$$

It is a consequence of the argument below that $I(Z(2, l))$ is actually equal to the ideal in (96)), but we do not need this result. We only verify that the generators displayed in (96) do indeed vanish on $Z(2, l)$. This is clear for the generators in the first term, which is the special case of (70) for $n=2$. The determinants in the second term vanish because on every $W_{f}$, either $f(i)=f(j)$, making the first two rows equal, or $f(i) \neq f(j)$, making one of the first two rows equal to the last. From (96) it is easy to see that the ideal

$$
I=I(Z(2, l))+\left(x_{1}\right)+(\mathbf{y})
$$

contains $a_{i}^{2}-a_{i} x_{2}, a_{i} b_{i}, b_{i}^{2}$ and $x_{2} b_{i}$ for all $i$, and $a_{j} b_{i}-a_{i} b_{j}$ for all $i<j$.

With respect to a suitable term ordering, the initial ideal of $I$ contains $a_{i}^{2}, a_{i} b_{i}$, $b_{i}^{2}$ and $x_{2} b_{i}$ for all $i$, and $a_{j} b_{i}$ for all $i<j$. Hence $S /\left(x_{1}\right)=k[\mathbf{x}, \mathbf{y}, \mathbf{a}, \mathbf{b}] / I$ is spanned by monomials in $k\left[x_{2}, \mathbf{a}, \mathbf{b}\right]$ not divisible by any of these. In other words, $S /\left(x_{1}\right)$ is spanned by monomials

$$
x_{2}^{k} \prod_{i \in S} a_{i} \prod_{j \in T} b_{j},
$$

where every element of $S$ is less than every element of $T$, and $k=0$ if $T \neq \emptyset$. Let us order the monomials in (98) so that those with smaller values of $k$ precede those with larger values, and for $k=0$, those that don't contain $b_{1}$ as a factor precede those that do. Then it is easy to see that each monomial in (98) occurs as the leading term in the reduction of an element of $B_{0}$ modulo $I$. This implies that $B_{0}$ spans $S /\left(x_{1}\right)$, as desired.

We now obtain the special case of Theorem 4.2 for $n=2$.

Lemma 4.6.2. For $n=2$, each ideal $I(m, r, k) \subseteq R(2, l)$ is spanned as a $k[\mathbf{y}]$ module by the set of elements $p[e, f] \in B$ indexed by $e, f$ satisfying

$$
\left|[r] \backslash S_{k}(e, f)\right|<m
$$

where $S_{k}(e, f)=\left\{j: e_{j}>0\right\} \cup f([k])$. 
Proof. First we verify that the specified elements do belong to $I(m, r, k)$. Observe (glancing ahead) that for each $m, r, k$, the ideal $I$ displayed on the right-hand side in (100) is generated by polynomials which vanish on $Y(m, r, k)$, so we have $I \subseteq I(m, r, k)$. It is mostly routine now to check case-by-case that the relevant elements $p[e, f]$ belong to $I$. The only tricky case is to show that $p[e, f]$ belongs to $I(1,2, k)$ for $e=(1,0), f(1)=2$, and $k>0$. In this case $p[e, f]=x_{1} \theta p[(0,0), \theta f]$ contains a factor $x_{1}\left(b_{1}-y_{1}\right)$, which is not so obviously in $I$. However, in this case $I$ contains $a_{1}-x_{1}-x_{2}$, and in $R(2, l)$ we have $\left(a_{1}-x_{2}\right)\left(b_{1}-y_{1}\right)=0$, so $x_{1}\left(b_{1}-y_{1}\right)=-\left(a_{1}-x_{1}-x_{2}\right)\left(b_{1}-y_{1}\right)$.

Using Lemma 4.4.11, to complete the proof it is enough to show that the specified elements $p[e, f] \operatorname{span} k(\mathbf{y}) \otimes I(m, r, k)$. Since $B$ is a homogeneous basis of $k(\mathbf{y}) \otimes R(2, l)$, it is equivalent to show that pairs $e, f$ not satisfying (99), counted according to the $x$-degree $|e|$ of $p[e, f]$, are enumerated by the Hilbert series of $k(\mathbf{y}) \otimes \mathcal{O}(Y(m, r, k))$. This is true by Corollary 4.5.5

Corollary 4.6.3. In the case $n=2$, the (nontrivial) ideals $I(m, r, k)$ are generated as ideals in $R(2, l)$ as follows:

$$
\begin{aligned}
& I(2,2,0)=(\mathbf{x}, \mathbf{a}), \\
& I(1,2, k)=\left(x_{1} x_{2}\right)+\sum_{i \in[k]}\left(a_{i}-x_{1}-x_{2}, b_{i}-b_{1}\right), \\
& I(1,1, k)=\left(x_{1}\right)+\sum_{i \in[k]}\left(a_{i}-x_{2}, b_{i}-y_{2}\right) .
\end{aligned}
$$

Proof. In each case the ideal listed on the right-hand side is clearly contained in $I(m, r, k)$. Conversely, in the proof of Lemma 4.6.2 we showed that $I(m, r, k)$ is generated (and even spanned as a $k[\mathbf{y}]$-module) by elements belonging to the ideal on the right-hand side.

Knowing the ideals $I(m, r, k)$ for $n=2$, we now want to draw conclusions for general $n$. The lemmas expressing our conclusions will come in pairs. In each pair we first establish a fact about the case $n=2$, then deduce its analog on $U_{2}$ for general $n$ by local reduction. Our first pair of lemmas extends the local reducedness result in Corollary 4.4.5 from $U_{1}$ to $U_{2}$. In this instance we will need the first lemma in the pair again later, for the proof of Lemma 4.9.5 (looking ahead, the reader may notice that Lemmas 4.9.4 and 4.9.5 form another pair of the same type).

Lemma 4.6.4. Fix a two-element subset $N \subseteq[n]$. Let $R_{1}$ be the coordinate ring of $E^{[n] \backslash N}$, so $R=R_{1} \otimes_{k} R(N, l)$ is the coordinate ring of $E^{[n] \backslash N} \times Z(N, l)$. Let $\mathcal{L}$ be the lattice of ideals in $R$ generated by the ideals $\left(x_{j}\right)$ for $j \notin N$ and $R_{1} \otimes I_{N, l}(m, r, k)$ for all $m, r, k$. Then $I=\sqrt{ } I$ for all $I \in \mathcal{L}$.

Proof. Let $\mathbf{x}^{\prime}, \mathbf{y}^{\prime}$ be the coordinates in indices $[n] \backslash N$, so $R_{1}=k\left[\mathbf{x}^{\prime}, \mathbf{y}^{\prime}\right]$. Obviously $R_{1}$ is a free $k\left[\mathbf{y}^{\prime}\right]$-module with basis $B_{1}$ the set of all monomials in the variables $\mathbf{x}^{\prime}$, and each ideal $\left(x_{j}\right) \cap R_{1}$ is spanned by a subset of $B_{1}$. By Lemma 4.6.2 $R_{2}=R(N, l)$ is a free $k\left[\mathbf{y}_{N}\right]$-module with a basis $B_{2}$ which is a common basis for the ideals $I_{N, l}(m, r, k)$. It follows that $R$ is a free $k[\mathbf{y}]$-module with basis $B_{1} \otimes B_{2}$ and that every ideal in $\mathcal{L}$ is spanned by a subset of $B_{1} \otimes B_{2}$. This implies that $\mathcal{L}$ is a distributive lattice, and hence every ideal in $\mathcal{L}$ is an intersection of ideals of the form $J=\left(x_{j}: j \in T\right) \otimes R_{2}+R_{1} \otimes I$, where $I$ is a sum of ideals $I_{N, l}(m, r, k)$. Now $V(J)=V\left(x_{j}: j \in T\right) \times V(I)$, so we only have to show that $V(I)$ is reduced. 
Lemma 4.6 .2 implies that $R_{2} / I$ is a free $k\left[\mathbf{y}_{N}\right]$-module, and hence a subring of its localization $\left(R_{2} / I\right)_{Q}$ at any point $Q \in \operatorname{Spec} k\left[\mathbf{y}_{N}\right]$. By Corollary 4.4.5 the latter ring is reduced for $Q \in \hat{U}_{1}$ (in particular, $Q=0$ suffices).

Lemma 4.6.5. If I belongs to the lattice generated by the ideals $I(m, r, k)$ in $R(n, l)$, then $V(I) \cap U_{2}$ is reduced.

Proof. We already have the result on $U_{1}$ by Corollary 4.4.5. Let $P$ be a point of $U_{2} \backslash U_{1}$. By Lemma 4.4.7, $Z(n, l)$ coincides locally at $P$ with a subspace arrangement isomorphic to $E^{[n] \backslash N} \times Z(N, L)$, for a set $N$ with two elements. Unraveling the definitions of $Y(m, r, k)$ and the local isomorphism, we find that $Y(m, r, k)$ coincides locally, as a reduced closed subscheme of $E^{[n] \backslash N} \times Z(N, L)$, with a subspace arrangement

$$
\bigcup_{T_{1}, m^{\prime}} V\left(x_{j}: j \in T_{1}\right) \times Y_{N, L}\left(m^{\prime}, r^{\prime}, k^{\prime}\right) .
$$

Here we set $r^{\prime}=|[r] \cap N|$ and $k^{\prime}=|[k] \cap L|$. The union ranges over $T_{1} \subseteq[n] \backslash N$ and $m^{\prime}$ satisfying $m^{\prime}+\left|T_{1} \cap([r] \backslash N) \backslash h([k])\right| \geq m$, where $h$ is chosen so that $P \in W_{h}$. Note that the set $([r] \backslash N) \backslash h([k])$ does not depend on the choice of $h$. It follows from Lemma 4.6.4 that the ideals of the subspace arrangements in (101), for $N, L$ fixed and $m, r, k$ arbitrary, belong to a lattice consisting entirely of radical ideals.

We remark that once Theorem 4.2 is established, we can conclude a posteriori that the lattice $\mathcal{L}$ generated by the ideals $I(m, r, k)$ is distributive, and that the ring $R(n, l) / I$ is a free $k[\mathbf{y}]$-module for every $I \in \mathcal{L}$. In fact, it can be shown that the existence of a common free module basis is equivalent to these conditions, plus the freeness of $R(n, l)$ itself. Combined with Corollary 4.4.5, the freeness of $R(n, l) / I$ for $I \in \mathcal{L}$ implies that $V(I)$ is reduced everywhere, not just on $U_{2}$, or in other words, $I=\sqrt{ } I$. However, we do not see a way to obtain these stronger results without appealing to Theorem 4.2. We must therefore content ourselves for now with the local result on $U_{2}$, and we will ultimately prove Theorem 4.2 for general $n$ by means that require only this local information.

We conclude our study of the case $n=2$ with another pair of lemmas giving a reducedness property for $n=2$ and the corresponding local property on $U_{2}$ for all $n$. For this (and again later) we need a preliminary lemma. By construction, the subspaces $W_{f}$ are invariant with respect to simultaneous translation of the $\mathbf{x}$ and a coordinates by a common quantity. This fact has the following consequence.

Lemma 4.6.6. Let $R$ be the coordinate ring of any union of the subspaces $W_{f} \subseteq$ $E^{n} \times E^{l}$. If $x$ is one of the coordinate variables $\mathbf{x}$, a, we have $(x)=\sqrt{ }(x)$ in $R$.

Proof. A manifestation of translation invariance is that the endomorphism $\tau_{x}$ of $k[\mathbf{x}, \mathbf{y}, \mathbf{a}, \mathbf{b}]$ defined by the substitutions

$$
x_{j} \mapsto x_{j}-x, \quad a_{i} \mapsto a_{i}-x, \quad \text { for all } j \in[n], i \in[l],
$$

carries the ideals $I_{f}$ into themselves, as is clear from their definition in (67). We therefore have $\tau_{x} I \subseteq I$, where $I$ is the defining ideal of $R=k[\mathbf{x}, \mathbf{y}, \mathbf{a}, \mathbf{b}] / I$. Since $\tau_{x}(x)=0$, we have an induced ring homomorphism $\tau_{x}: R /(x) \rightarrow R$. We see immediately that if $\phi: R \rightarrow R /(x)$ is the canonical projection, then $\phi \circ \tau_{x}$ is the identity map on $R /(x)$. Hence $R /(x)$ is isomorphic to a subring of $R$. Since $R$ is reduced, so is $R /(x)$, so we have $(x)=\sqrt{ }(x)$. 
We will need the second lemma of the following pair for the proof of Lemma 4.10.7.

Lemma 4.6.7. For $n=2$ and $t \in[l]$ the ideal $I=I(1,1, t-1)+\left(a_{t}\right)$ in $R(2, l)$ is equal to its radical.

Proof. By Corollary 4.6.3, we have $I=J+\left(x_{1}, a_{t}\right)$, where $J=\sum_{i<t}\left(a_{i}-x_{2}, b_{i}-y_{2}\right)$. By Lemma 4.4.8, we have $R(2, l) / J \cong R(2, L)$ where $L=[l] \backslash[t-1]$. Replacing [l] with $L$, this reduces the problem to showing that $I=\sqrt{ } I$ in $R(2, l)$ for $I=\left(x_{1}, a_{1}\right)$. For this $I$ we have set-theoretically

$$
V(I)=\left(\bigcup_{f(1)=1} V\left(x_{1}\right) \cap W_{f}\right) \cup\left(\bigcup_{f(1)=2} V\left(x_{1}, x_{2}\right) \cap W_{f}\right) .
$$

By Lemma 4.4.8, the ideal $\left(a_{1}-x_{1}, b_{1}-y_{1}\right)$ is equal to its radical, and its zero set is $\bigcup_{f(1)=1} W_{f}$. Applying Lemma 4.6.6 with $x=x_{1}$ to the union of subspaces $\bigcup_{f(1)=1} W_{f}$, we see that the ideal $\left(x_{1}\right)+\left(a_{1}-x_{1}, b_{1}-y_{1}\right)=I+\left(b_{1}-y_{1}\right)$ is equal to its radical, and hence to $(\sqrt{ } I)+\left(b_{1}-y_{1}\right)$. The zero set of this ideal is $V\left(x_{1}\right) \cap \bigcup_{f(1)=1} W_{f}$, which is the first big union in (103).

Again by Lemma 4.4.8, the ideal $\left(a_{1}-x_{2}, b_{1}-y_{2}\right)$ is equal to its radical, with zero set $\bigcup_{f(1)=2} W_{f} \cong Z(2, l-1)$. Observing that $V\left(x_{1}, x_{2}\right)=Y(2,2,0)$ in $Z(2, l-1)$, it follows from Corollary 4.6.3 that the ideal of $V\left(x_{1}, x_{2}\right) \cap \bigcup_{f(1)=2} W_{f}$, that is, of the second big union in (103), is $(\mathbf{x}, \mathbf{a})+\left(a_{1}-x_{2}, b_{1}-y_{2}\right)=(\mathbf{x}, \mathbf{a})+\left(b_{1}-y_{2}\right)$. Since $b_{1}-y_{1}$ does not vanish identically on any component of the second big union, the ideal $(\sqrt{ } I):\left(b_{1}-y_{1}\right)$ is equal to the ideal of the latter. Hence we have $(\sqrt{ } I):\left(b_{1}-y_{1}\right)=$ $(\mathbf{x}, \mathbf{a})+\left(b_{1}-y_{2}\right)$.

We now claim that $I:\left(b_{1}-y_{1}\right)$ contains $(\mathbf{x}, \mathbf{a})+\left(b_{1}-y_{2}\right)$, and hence $I:\left(b_{1}-y_{1}\right)=$ $(\sqrt{ } I):\left(b_{1}-y_{1}\right)$. For the claim, observe that $\left(b_{1}-y_{1}\right)\left(b_{1}-y_{2}\right)=0$ in $R(2, l)$, which shows that $b_{1}-y_{2} \in I:\left(b_{1}-y_{1}\right)$. Observe also that $\left(b_{1}-y_{1}\right)\left(a_{1}-x_{2}\right)=0$ in $R(2, l)$, which shows that $x_{2} \in I:\left(b_{1}-y_{1}\right)$. Finally observe that the determinant

$$
\operatorname{det}\left[\begin{array}{lll}
a_{i} & b_{i} & 1 \\
a_{1} & b_{1} & 1 \\
x_{1} & y_{1} & 1
\end{array}\right]
$$

from (96) reduces modulo $\left(x_{1}, a_{1}\right)$ to $a_{i}\left(b_{1}-y_{1}\right)$, which shows that $a_{i} \in I:\left(b_{1}-y_{1}\right)$ for all $i$.

Multiplying the identity of ideals $I:\left(b_{1}-y_{1}\right)=(\sqrt{ } I):\left(b_{1}-y_{1}\right)$ by $b_{1}-y_{1}$ yields $I \cap\left(b_{1}-y_{1}\right)=(\sqrt{ } I) \cap\left(b_{1}-y_{1}\right)$, and we showed above that $I+\left(b_{1}-y_{1}\right)=$ $(\sqrt{ } I)+\left(b_{1}-y_{1}\right)$. By the modular law for ideals, since $I \subseteq \sqrt{ } I$, these imply $I=\sqrt{ } I$.

Lemma 4.6.8. For all $n$ and for $t \in[l]$, if $I$ is the ideal $I(1,1, t-1)+\left(a_{t}\right)$ in $R(n, l)$, then $V(I) \cap U_{2}$ is reduced.

Proof. Note that by Lemma 4.6.6, $V\left(a_{t}\right)$ is reduced and equal to the union over all $f$ of $V\left(x_{f(t)}\right) \cap W_{f}$. Thus $\left(a_{t}\right)$ belongs to the lattice of ideals in Lemma 4.4.4 as does $I(1,1, t-1)$. Therefore $V(I) \cap U_{1}$ is reduced.

For $P \in U_{2} \backslash U_{1}, Z(n, l)$ coincides locally with the arrangement $Z=E^{[n] \backslash N} \times$ $Z(N, L)$ from Lemma 4.4.7 and we may as well replace $Z(n, l)$ with $Z$. 
First suppose $1 \notin N$. Fixing some $h$ such that $P \in W_{h}$ as in Lemma 4.4.7 either $Y(1,1, t-1)$ is locally empty, if $1 \in h([t-1])$, or else $Y(1,1, t-1)$ coincides locally with $V\left(x_{1}\right)$. If $t \notin L$, then $V\left(a_{t}\right)=V\left(x_{j}\right)$ for $j=h(t) \notin N$. Otherwise $V\left(a_{t}\right)=E^{[n] \backslash N} \times Y$, where $Y=V\left(a_{t}\right) \cap Z(N, L)$, which is reduced by Lemma4.6.6 In every case, the scheme-theoretic intersection of $Y(1,1, t-1)$ and $V\left(a_{t}\right)$ is either empty or coincides locally with a product of reduced schemes, so $V(I)$ is locally reduced when $1 \notin N$.

Suppose instead that $1 \in N$. Then $Y(1,1, t-1)$ coincides locally with $E^{[n] \backslash N} \times$ $Y\left(1,1, t^{\prime}-1\right)$, where $t^{\prime}-1=|[t-1] \cap L|$. If $t \notin L$ we have $V\left(a_{t}\right)=V\left(x_{h(t)}\right)$ and the result is immediate, as before. Otherwise it reduces to Lemma 4.6.7

4.7. Further description of $Y(m, r, k)$. In $\S \$ 4.9$ and 4.10 we will prove a series of lemmas enabling us to extend bases of the type specified in Theorem 4.2 from the coordinate rings of smaller arrangements to those of larger ones. Since the characteristic property of our bases is their compatibility with the ideals $I(m, r, k)$, we will of course have to use some geometric facts about the corresponding subspace arrangements $Y(m, r, k)$. Our purpose in this subsection is to take note of some such facts for later use. We begin by refining our description of the arrangements $Y(m, r, k)$.

Lemma 4.7.1. Let $P$ be a point of $Z(n, l)$ and let $f$ be the pointwise maximum of all functions $h$ such that $P$ lies on $W_{h}$. Then $P$ lies on $W_{f}$, and $P$ belongs to $Y(m, r, k)$ if and only if the set $T=\left\{j: P \in V\left(x_{j}\right)\right\}$ satisfies $|T \cap[r] \backslash f([k])| \geq m$.

Proof. Define an equivalence relation $\sim_{P}^{\prime}$ on $[n]$ by the rule $i \sim_{P}^{\prime} j$ if and only if $P \in V\left(x_{i}-x_{j}, y_{i}-y_{j}\right)$. It induces an equivalence relation $\sim_{P}$ on functions $h:[l] \rightarrow[n]$ by the rule $g \sim_{P} h$ if and only if $g(i) \sim_{P}^{\prime} h(i)$ for all $i \in[l]$ (this differs from the equivalence relation in Lemma 4.4.7 in that it depends on both the $\mathbf{x}$ and the $\mathbf{y}$ coordinates). It is easy to see that the functions $h$ such that $P$ lies on $W_{h}$ form an equivalence class, and that the pointwise maximum of two equivalent functions is equivalent to each of them. This shows that $P$ lies on $W_{f}$.

The function $f$ takes at most one value in each $\sim_{P}^{\prime}$-equivalence class, namely, the maximum element in that class. Furthermore, that value is only in $[r]$ if the whole equivalence class is contained in $[r]$. Hence, among all functions in its $\sim_{P^{-}}$ equivalence class, $f$ maximizes the size of the intersection of $[r] \backslash f([k])$ with every $\sim_{P}^{\prime}$-equivalence class. The set $T$ is a union of $\sim_{P^{-}}^{\prime}$ equivalence classes, so it follows that $f$ maximizes $|T \cap[r] \backslash f([k])|$. But $P$ belongs to $Y(m, r, k)$ if and only if the maximum of this quantity over all $h$ such that $P \in W_{h}$ is at least $m$.

At certain points later on we will need to know that intersections of the arrangements $Y(m, r, k)$ consist of spaces on which the $\mathbf{y}$ coordinates are independent. Note that this is not obvious from the definition, although it has to be true if Theorem 4.2 is to hold. Using the preceding lemma, we can prove a precise version of the fact we require.

Lemma 4.7.2. Set-theoretically, $Y(m, r, k) \cap Y\left(m^{\prime}, r^{\prime}, k^{\prime}\right)$ is the union of subspaces

$$
\bigcup_{f, T} V\left(x_{j}: j \in T\right) \cap W_{f}
$$

over all $f:[l] \rightarrow[n]$ and $T \subseteq[n]$ satisfying

$$
|T \cap[r] \backslash f([k])| \geq m \text { and }\left|T \cap\left[r^{\prime}\right] \backslash f\left(\left[k^{\prime}\right]\right)\right| \geq m^{\prime} .
$$


Proof. It is clear that $Y(m, r, k) \cap Y\left(m^{\prime}, r^{\prime}, k^{\prime}\right)$ contains the union in (105). Given a point $P$ of $Y(m, r, k) \cap Y\left(m^{\prime}, r^{\prime}, k^{\prime}\right)$, let $T=\left\{j: P \in V\left(x_{j}\right)\right\}$. By the definitions of $Y(m, r, k)$ and $Y\left(m^{\prime}, r^{\prime}, k^{\prime}\right)$, we have $P \in W_{f}$ for some $f$ such that $|T \cap[r] \backslash f([k])| \geq$ $m$, and $P \in W_{f^{\prime}}$ for some $f^{\prime}$ such that $\left|T \cap\left[r^{\prime}\right] \backslash f^{\prime}\left(\left[k^{\prime}\right]\right)\right| \geq m^{\prime}$. A priori, $f$ and $f^{\prime}$ might be different, but Lemma 4.7 .1 allows us to take them both to be the maximum $f$ for which $W_{f}$ contains $P$. Hence $Y(m, r, k) \cap Y\left(m^{\prime}, r^{\prime}, k^{\prime}\right)$ is contained in the union in (105).

In the proof of Lemma 4.10 .7 we will need one more result. To state it we need to introduce the circular shift permutation $\theta=\left(\begin{array}{lll}1 & 2 & \ldots\end{array}\right)$, acting on $E^{n} \times E^{l}$ as the automorphism which shifts the $E^{n}$ coordinates and fixes the $E^{l}$ coordinates, that is,

$$
\theta\left(P_{1}, \ldots, P_{n}, Q_{1}, \ldots, Q_{l}\right)=\left(P_{n}, P_{1}, \ldots, P_{n-1}, Q_{1}, \ldots, Q_{l}\right) .
$$

Since the definition of the polygraph is symmetric in the $P_{i}$ 's, $\theta$ maps $Z(n, l)$ onto itself.

Lemma 4.7.3. Given $n, l$ and $t \in[l]$, set $L=[l] \backslash\{t\}$ and let $\pi: Z(n, l) \rightarrow Z(n, L)$ be the projection on the coordinates other than $a_{t}, b_{t}$. Let $\theta$ be as above. Then for $r>0$ and $k<t$ we have

$$
\begin{aligned}
& Y(1,1, t) \cup(Y(m, r, k) \cap Y(1,1, t-1)) \\
& \quad=Y(1,1, t) \cup\left(\pi^{-1} \theta Y_{n, L}(m-1, r-1, k) \cap Y(1,1, t-1)\right),
\end{aligned}
$$

while for $r>0$ and $k \geq t$ we have

$$
\begin{aligned}
& Y(1,1, t) \cup(Y(m, r, k) \cap Y(1,1, t-1)) \\
& \quad=Y(1,1, t) \cup\left(\pi^{-1} \theta Y_{n, L}(m, r-1, k-1) \cap Y(1,1, t-1)\right) .
\end{aligned}
$$

Proof. For each identity, we are to show that a point $P$ belongs to the set on the left-hand side if and only if it belongs to the set on the right-hand side. Suppose first that we have $P \in V\left(x_{1}-x_{j}, y_{1}-y_{j}\right)$ for some $j>1$. In the notation of the proof of Lemma 4.7.1, this means that $1 \sim_{P}^{\prime} j$. In this case, we have $1 \notin f([l])$, where $f$ is the pointwise maximum function for which $P \in W_{f}$. From the criterion in Lemma 4.7.1 we see that $P \in Y(1,1, t-1)$ if and only if $P \in Y(1,1, t)$. Hence both identities (108) and (109) hold trivially for such a point $P$. We may therefore assume that $P \notin V\left(x_{1}-x_{j}, y_{1}-y_{j}\right)$ for all $j>1$. Then the set $S=h^{-1}(\{1\})$ is the same for every function $h$ such that $P \in W_{h}$. If we have $P \in Y(1,1, t-1)$ and $P \notin Y(1,1, t)$, then $t$ must be the least element of $S$, and we must have $P \in V\left(x_{1}\right)$. Thus we may assume that these conditions hold.

For $k<t$, we are to show that $P \in Y(m, r, k)$ if and only if

$$
\pi(P) \in \theta Y_{n, L}(m-1, r-1, k),
$$

assuming the above conditions. Let $f$ be the pointwise maximum function for which $P \in W_{f}$. Note that a component $W_{h} \subseteq Z(n, L)$ contains $\pi(P)$ if and only if $h=\left.g\right|_{L}$ for some $W_{g}$ containing $P$. Hence $h=\left.f\right|_{L}$ is the pointwise maximum $h$ such that $W_{h}$ contains $\pi(P)$. We claim that $h$ is also pointwise maximum for the circularly shifted ordering $2<3<\cdots<n<1$ of $[n]$. To see this, suppose $h^{\prime}$ is the maximum for this ordering. Clearly $h^{\prime}(i)$ can only differ from $h(i)$ if $h^{\prime}(i)=1$. But that would force $1 \sim_{P}^{\prime} h(i)$, contrary to our assumptions. It follows that we can test 
$\pi(P)$ for membership in $\theta Y_{n, L}(m-1, r-1, k)$ by applying the criterion in Lemma 4.7.1 using the function $h$.

Let $T=\left\{j: P \in V\left(x_{j}\right)\right\}$. By Lemma4.7.1 we have $P \in Y(m, r, k)$ if and only if $|T \cap[r] \backslash f([k])| \geq m$. By assumption, we have $1 \in T$ and $1 \notin f([t-1])$. Hence, since $k<t$, we have $1 \in T \cap[r] \backslash f([k])$ and $|T \cap[r] \backslash f([k])|=1+|T \cap([r] \backslash\{1\}) \backslash f([k])|$. Abusing notation, we write $\theta$ for the circular shift $\left(\begin{array}{l}1 \\ 2 \ldots n\end{array}\right)$ permuting the index set $[n]$ as well as for the corresponding automorphism of $Z(n, L)$. Then we have $[r] \backslash\{1\}=\theta[r-1]$. Since $k<t$, we also have $f([k])=h([k])$. Thus we have $P \in Y(m, r, k)$ if and only if $|T \cap \theta[r-1] \backslash h([k])| \geq m-1$. By Lemma 4.7.1 this is precisely the criterion for $\pi(P) \in \theta Y_{n, L}(m-1, r-1, k)$, which establishes (108).

For $k \geq t$, we are to show that $P \in Y(m, r, k)$ if and only if

$$
\pi(P) \in \theta Y_{n, L}(m, r-1, k-1) .
$$

As before, we can test the latter condition using the criterion in Lemma 4.7.1 with the function $h$. By assumption we have $f(t)=1$. Hence we have $1 \notin T \cap[r] \backslash f([k])$ and $|T \cap[r] \backslash f([k])|=|T \cap([r] \backslash\{1\}) \backslash f([k])|=|T \cap \theta[r-1] \backslash h([k] \backslash\{t\})|$. Now we conclude as before that $P$ belongs to $Y(m, r, k)$ if and only if $\pi(P)$ belongs to $\theta Y_{n, L}(m, r-1, k-1)$.

4.8. Lifting common ideal bases. We now develop a technique for lifting a common free $k[\mathbf{y}]$-module basis for certain ideals in a $k[\mathbf{y}]$-algebra $R$ to a basis of a finite $R$-algebra $S$ generated by one extra coordinate variable $b$. We give the general method here. In $\$ 4.9$ we apply it with $R=R(n, l-1)$ to obtain bases for the coordinate rings of certain special arrangements $Z^{\prime}$ contained in $Z(n, l)$. This basis lifting from $Z(n, l-1)$ to $Z^{\prime} \subseteq Z(n, l)$ will be crucial for one of the three stages of our basis construction procedure in 4.10

Definition 4.8.1. Let $Z=\operatorname{Spec} R$ be a Noetherian affine scheme. Let $S=R[b] / J$ be a finite $R$-algebra generated by one variable b, i.e., $Z^{\prime}=\operatorname{Spec} S$ is a closed subscheme of $\mathbb{A}^{1}(Z)$ finite over $Z$. We denote by $I_{m}\left(Z^{\prime}\right)$ the ideal in $R$ consisting of all elements $a_{m-1}$ for some

$$
a_{m-1} b^{m-1}+\cdots+a_{1} b+a_{0} \in J \quad\left(a_{i} \in R\right) .
$$

We denote by $V_{m}\left(Z^{\prime}\right)$ the corresponding closed subscheme $V\left(I_{m}\left(Z^{\prime}\right)\right) \subseteq Z$.

Note that the elements in (112) for a given $m$ constitute an $R$-submodule of $J$, so $I_{m}\left(Z^{\prime}\right)$ is in fact an ideal. If $F_{m}$ denotes the $R$-submodule of $S$ generated by the elements $\left\{1, b, \ldots, b^{m-1}\right\}$, then $F_{m} / F_{m-1}$ is a cyclic $R$-module generated by $b^{m-1}$ and isomorphic to $R / I_{m}\left(Z^{\prime}\right)$. In other words, we have $R b^{m-1} \cap F_{m-1}=$ $b^{m-1} I_{m}\left(Z^{\prime}\right)$. Since $S$ is assumed to be finitely generated as an $R$-module, we have $F_{r}=S$ for some $r$ and hence $I_{m}\left(Z^{\prime}\right)=(1)$ for all $m>r$.

Multiplying (112) by $b$, we see that $I_{m}\left(Z^{\prime}\right) \subseteq I_{m+1}\left(Z^{\prime}\right)$ for all $m$. If we agree to set $I_{0}\left(Z^{\prime}\right)=0$, then we have

$$
0=I_{0}\left(Z^{\prime}\right) \subseteq I_{1}\left(Z^{\prime}\right) \subseteq \cdots \subseteq I_{r+1}\left(Z^{\prime}\right)=(1) .
$$

The ideals $I_{m}\left(Z^{\prime}\right)$ are called partial elimination ideals by Green [20], who studied them in connection with the theory of lexicographic generic initial ideals. Green observed that the geometric interpretation of the loci $V_{m}\left(Z^{\prime}\right)$ has to do with the lengths of the fibers of the morphism $Z^{\prime} \rightarrow Z$, as we now explain. 
Definition 4.8.2. Let $S$ be a finitely generated module over a Noetherian commutative ring $R$. For $P \in \operatorname{Spec} R$ we set

$$
\mu_{P}(S)=\operatorname{dim}_{K_{P}}\left(K_{P} \otimes_{R} S\right),
$$

where $K_{P}=R_{P} / P R_{P}$ is the residue field of the local ring at $P$.

By Nakayama's lemma, $\mu_{P}(S)$ is the number of elements of any minimal set of generators of $S_{P}$ as an $R_{P}$-module. Also, if $S$ is an $R$-algebra, then $\mu_{P}(S)$ is the length of the fiber of Spec $S$ over the $K_{P}$-valued point $\operatorname{Spec} K_{P} \rightarrow \operatorname{Spec} R$ with image $P$.

Lemma 4.8.3 (20]). Let $Z$ and $Z^{\prime}$ be as in Definition 4.8.1. As sets, we have

$$
V_{m}\left(Z^{\prime}\right)=\left\{P \in Z: \mu_{P}(S) \geq m\right\}
$$

Proof. Let $P$ be a point of $Z=\operatorname{Spec} R$. The Artin $K_{P}$-algebra $K_{P} \otimes_{R} S$ is a quotient of the polynomial ring $K_{P}[b]$, and therefore has a $K_{P}$-basis $\left\{1, b, \ldots, b^{m_{0}-1}\right\}$, where $m_{0}=\mu_{P}(S)$. Equivalently, $\left\{1, b, \ldots, b^{m_{0}-1}\right\}$ minimally generates $S_{P}$ as an $R_{P^{-}}$ module, so with $F_{m}$ as in the remarks following Definition 4.8.1, we have $\left(F_{m_{0}}\right)_{P}=$ $S_{P},\left(F_{m_{0}-1}\right)_{P} \neq S_{P}$. This implies $I_{m_{0}}\left(Z^{\prime}\right)_{P} \neq(1), I_{m_{0}+1}\left(Z^{\prime}\right)_{P}=(1)$, that is, $P \in V_{m_{0}}\left(Z^{\prime}\right), P \notin V_{m_{0}+1}\left(Z^{\prime}\right)$. By (113) it follows that $P \in V_{m}\left(Z^{\prime}\right)$ if and only if $m \leq \mu_{P}(S)$.

The next two lemmas give some further elementary facts about partial elimination ideals.

Lemma 4.8.4. Let $Z$ and $Z^{\prime}$ be as in Definition 4.8.1. Assume they are schemes over $k$, and let $X$ be an affine scheme flat over $k$. Then we have

$$
V_{m}\left(Z^{\prime} \times X\right)=V_{m}\left(Z^{\prime}\right) \times X
$$

as closed subschemes of $Z \times X$.

Proof. Let $Z=\operatorname{Spec} R, Z^{\prime}=\operatorname{Spec} S$ and $X=\operatorname{Spec} T$, where $S=R[b] / J$. Then $S \otimes T=(R \otimes T)[b] /(J \otimes T)$, so the defining ideal of $Z^{\prime} \times X$ is $J \otimes T$. Since $T$ is flat, we have $(J \otimes T) \cap(R \otimes T)\left\{1, b, \ldots, b^{m-1}\right\}=\left(J \cap R\left\{1, b, \ldots, b^{m-1}\right\}\right) \otimes T$. From the definition it then follows that $I_{m}\left(Z^{\prime} \times X\right)=I_{m}\left(Z^{\prime}\right) \otimes T$, which is the algebraic equivalent of (116).

Note that the lemma as stated holds over an arbitrary ground ring $k$. When $k$ is a field the hypothesis that $X$ is flat over $k$ is satisfied automatically.

Lemma 4.8.5. With $Z$ and $Z^{\prime}$ as in Definition 4.8.1. suppose that $Z^{\prime}$ is the scheme-theoretic union of closed subschemes $Z_{1}^{\prime}$ and $Z_{2}^{\prime}$, that is, the defining ideal of $Z^{\prime}$ is an intersection $J=J_{1} \cap J_{2}$. Then we have

$$
I_{s}\left(Z_{1}^{\prime}\right) I_{t}\left(Z_{2}^{\prime}\right) \subseteq I_{s+t-1}\left(Z^{\prime}\right)
$$

for all $s, t$.

Proof. Let $p \in I_{s}\left(Z_{1}^{\prime}\right)$ be the leading coefficient of an element $f \in J_{1}$ with leading form $p b^{s-1}$, as in (112). Similarly let $q \in I_{t}\left(Z_{2}^{\prime}\right)$ be the leading coefficient of $g \in J_{2}$ with leading form $q b^{t-1}$. Then we have $f g \in J_{1} J_{2} \subseteq J$, and the leading form of $f g$ is $p q b^{m-1}$ where $m=s+t-1$. 
For us, the main significance of the partial elimination ideals $I_{m}\left(Z^{\prime}\right)$ is their usefulness in lifting a free $k[\mathbf{y}]$-module basis from $R$ to $S$. Supposing that $R$ has a basis which is a common basis for all the partial elimination ideals, it is straightforward to construct from it a free $k[\mathbf{y}]$-module basis of $S$. In keeping with our program of reducing everything to the case $n=2$, we require a stronger form of the construction, in which the hypotheses are limited to things we can check locally on $U_{2}$ or $U_{1}$. We remark, however, that when the hypotheses in the following lemma hold locally, it actually follows that they hold everywhere - in other words, the ideals $I_{m}$ in (118) below are equal to the partial elimination ideals.

Lemma 4.8.6. Let $R$ be a $k[\mathbf{y}]$-algebra, and let $Z=\operatorname{Spec} R$ and $Z^{\prime}=\operatorname{Spec} S$ be as in Definition 4.8.1. Assume that $R$ is free and $S$ is torsion-free as $k[\mathbf{y}]$-modules. Suppose that $R$ has a basis $B$ which is a union of disjoint subsets $B=\bigcup_{j=0}^{r} B_{j}$ such that for each $m$, the submodule $I_{m}=k[\mathbf{y}] \bigcup_{j<m} B_{j}$ is an ideal in $R$ satisfying

$$
I_{m}\left(Z^{\prime}\right)_{P}=\left(I_{m}\right)_{P}
$$

for all $P \in U_{2} \cap Z$. Then $S$ is a free $k[\mathbf{y}]$-module with basis

$$
B^{\prime}=\bigcup_{m=1}^{r} B_{m} \cdot\left\{1, b, \ldots, b^{m-1}\right\} .
$$

Furthermore, let $I \subseteq R$ be an ideal spanned as a (free) $k[\mathbf{y}]$-module by a subset $A \subseteq B$, and let $Y^{\prime}=\operatorname{Spec} S / I S$. Suppose that

$$
I_{m}\left(Y^{\prime}\right)_{P}=I_{P}+I_{m}\left(Z^{\prime}\right)_{P}
$$

for all $m>0$ and all $P \in U_{1} \cap Z$. Then the ideal $I S$ is spanned by the subset $A^{\prime}=\left(A \cdot\left\{1, b, b^{2}, \ldots\right\}\right) \cap B^{\prime}$ of $B^{\prime}$.

Proof. Consider the filtration $0=F_{0} \subseteq F_{1} \subseteq \cdots$ of $S$, where $F_{m}=R \cdot\{1, b, \ldots$, $\left.b^{m-1}\right\}$. Without loss of generality we can assume that $F_{r}=S$, since the hypotheses and conclusion of the lemma are unaltered if we replace $r$ by a larger integer $r_{1}$ and define the extra subsets $B_{j}$ for $r<j \leq r_{1}$ to be empty. We have $F_{m} / F_{m-1} \cong$ $R / I_{m}\left(Z^{\prime}\right)$ and therefore, by (118) and Lemma 4.4.2 $\left(F_{m} / F_{m-1}\right)_{Q} \cong\left(R / I_{m}\right)_{Q}$ for all $Q \in \hat{U}_{2}$. It follows that $\left(F_{m} / F_{m-1}\right)_{Q}$ is a free $k[\mathbf{y}]_{Q}$-module with basis $b^{m-1} \bigcup_{j \geq m} B_{j}$, and since $B^{\prime}$ is the union of these, $S_{Q}$ is a free $k[\mathbf{y}]_{Q^{-m o d u l e}}$ with basis $B^{\prime}$. By Lemma 4.4.9 this implies that $S$ is a free $k[\mathbf{y}]$-module with basis $B^{\prime}$.

By (120) and Lemma 4.4.2, we have $I_{m}\left(Y^{\prime}\right)_{Q}=I_{Q}+I_{m}\left(Z^{\prime}\right)_{Q}$ for $Q \in \hat{U}_{1}$. By the definition of $Y^{\prime}$, multiplication by $b^{m-1}$ is an isomorphism

$$
R / I_{m}\left(Y^{\prime}\right) \rightarrow\left(F_{m}+I S\right) /\left(F_{m-1}+I S\right) \cong F_{m} /\left(F_{m-1}+I S \cap F_{m}\right) .
$$

The $R$-module $F_{m} /\left(F_{m-1}+b^{m-1} I\right)$ is generated by $b^{m-1}$ and is therefore equal to $R b^{m-1} /\left(R b^{m-1} \cap\left(F_{m-1}+b^{m-1} I\right)\right)$. By the modular law and the identity $R b^{m-1} \cap F_{m-1}=b^{m-1} I_{m}\left(Z^{\prime}\right)$, this is in turn equal to $R b^{m-1} /\left(b^{m-1} I_{m}\left(Z^{\prime}\right)+b^{m-1} I\right)$. Multiplication by $b^{m-1}$ is thus an isomorphism

$$
R /\left(I_{m}\left(Z^{\prime}\right)+I\right) \rightarrow F_{m} /\left(F_{m-1}+b^{m-1} I\right) .
$$

Hence for $Q \in \hat{U}_{1}$ we have $\left(F_{m-1}+I S \cap F_{m}\right)_{Q}=\left(F_{m-1}+b^{m-1} I\right)_{Q}$ and therefore $\left(I S \cap F_{m}\right)_{Q} \subseteq\left(b^{m-1} I+F_{m-1} \cap I S\right)_{Q}$. It follows that the map

$$
I_{Q} /\left(I_{m}\left(Z^{\prime}\right) \cap I\right)_{Q} \rightarrow\left(I S \cap F_{m}\right)_{Q} /\left(I S \cap F_{m-1}\right)_{Q}
$$


given by multiplication by $b^{m-1}$ is surjective. It is injective by the definition of $I_{m}\left(Z^{\prime}\right)$, so it is an isomorphism.

Setting $A_{j}=A \cap B_{j}$, this implies that $b^{m-1} \bigcup_{j \geq m} A_{j}$ is a basis of $\left(I S \cap F_{m}\right)_{Q} /$ $\left(I S \cap F_{m-1}\right)_{Q}$. Since $A^{\prime}$ is the union of these, $A^{\prime}$ is a basis of $I S_{Q}$. In particular this holds for $Q=0$, which by Lemma 4.4.11 suffices to show that $A^{\prime}$ spans $I S$.

4.9. Lifting for special arrangements. The most intricate stage in the basis construction procedure to be presented in 4.10 involves the construction of a free $k[\mathbf{y}]$-module basis in the ring $R(n, l) / I(1,1, t-1)$, given a suitable basis in the ring $R(n, l) / I(1,1, t)$. To pass from one ring to the other we will need a basis of $I(1,1, t) / I(1,1, t-1)$. We will get this basis from a basis of $R(n, l-1)$ by applying the lifting theory from 4.8 to certain special arrangements, which we now define.

Definition 4.9.1. Given $n, l, r$ and $k$, with $r \in[n] \cup\{0\}$ and $k \in[l]$, the special arrangement $Z^{\prime}=Z^{\prime}(r, k)$ is the subspace arrangement

$$
Z^{\prime}=\bigcup V\left(a_{k}\right) \cap W_{f}, \quad \text { over } f:[l] \rightarrow[n] \text { such that } f(k) \in[r] \backslash f([k-1]),
$$

a reduced closed subscheme of $Z(n, l)$. We denote by $\pi: Z^{\prime} \rightarrow Z(n, L)$ the projection on the coordinates other than $a_{k}, b_{k}$, mapping $Z^{\prime}$ into the polygraph $Z(n, L)$ in indices $[n]$ and $L=[l] \backslash\{k\}$.

The significance of the special arrangements $Z^{\prime}(r, k)$ is twofold. On the one hand, they are related to the arrangements $Y_{n, L}(m, r, k-1)$ in the ground scheme $Z(n, L)$ via our basis lifting theory. On the other hand, in $Z(n, l)$ there is a geometric relationship between the closed subsets $Z^{\prime}(n, t), Z^{\prime}(n-1, t), Y(1,1, t)$ and $Y(1,1, t-1)$ that makes the step from $R(n, l) / I(1,1, t-1)$ to $R(n, l) / I(1,1, t)$ possible. The latter aspect will be explained in detail in $\$ 4.10$ Here we treat the basis lifting aspect. To start with, we need the following purely set-theoretic fact, which already gives an idea of the relationship between $Z^{\prime}(r, k)$ and $Y_{n, L}(m, r, k-1)$.

Lemma 4.9.2. Let $Z^{\prime}=Z^{\prime}(r, k)$ be a special arrangement over $Z=Z(n, L)$, where $L=[l] \backslash\{k\}$, and let $\pi: Z^{\prime} \rightarrow Z(n, L)$ be the coordinate projection. Then $Y_{n, L}(m, r, k-1) \subseteq Z(n, L)$ is the closure of the locus consisting of points $P \in$ $U_{1} \cap Z(n, L)$ for which the fiber $\pi^{-1}(P)$ has size $\left|\pi^{-1}(P)\right| \geq m$.

Proof. Let $P$ be a point of $U_{1} \cap Z(n, L)$, and let $Q \in \pi^{-1}(P)$ be a point of the fiber. Note that this implies $Q \in U_{1} \cap Z(n, l)$. By Lemma 4.4.3 there is a unique $W_{f}$ containing $Q$ and a unique $W_{h}$ containing $P$. Clearly we must have $h=\left.f\right|_{L}$. By the definition of $Z^{\prime}$, we have $f(k) \in[r] \backslash h([k-1])$, and $x_{f(k)}$ vanishes at $Q$ and hence at $P$. Conversely, for every $j \in[r] \backslash h([k-1])$ such that $x_{j}$ vanishes at $P$, there is a point $Q \in \pi^{-1}(P)$ lying on $W_{f}$, where $f(k)=j$ and $\left.f\right|_{L}=h$. Note that $f$ determines $Q$, since the subspaces $W_{f} \subseteq E^{n} \times E^{l}$ and $W_{h} \subseteq E^{n} \times E^{L}$ both project isomorphically onto $E^{n}$, and the two projections map $Q$ and $P$ to the same point.

This shows that the number of points in the fiber $\pi^{-1}(P)$ is equal to the size of the set $T \cap[r] \backslash h([k-1])$, where $T=\left\{j: P \in V\left(x_{j}\right)\right\}$. By Lemma 4.7.1] $Y_{n, L}(m, r, k-1) \cap U_{1}$ consists of those points $P$ for which $|T \cap[r] \backslash h([k-1])| \geq m$. Since $Y_{n, L}(m, r, k-1) \cap U_{1}$ is dense in $Y_{n, L}(m, r, k-1)$, the result follows.

We will now apply the theory developed in $\$ 4.8$ to the case where $Z^{\prime}=\operatorname{Spec} S$ is the special arrangement $Z^{\prime}(r, k)$ over $Z=Z(n, L)$, with $L=[l] \backslash\{k\}$. The coordinate ring of the ground scheme is thus $R=R(n, L)$. The map $\pi: Z^{\prime} \rightarrow Z$ is the projection on the coordinates other than $a_{k}, b_{k}$. Note that $a_{k}$ vanishes on 
$Z^{\prime}$ by definition, so $Z^{\prime}$ is a closed subscheme of $\mathbb{A}^{1} \times Z(n, L)$, where the extra coordinate $b$ on $\mathbb{A}^{1}$ is $b_{k}$. Note also that the product $\prod_{j \in[n]}\left(b_{k}-y_{j}\right) \in R\left[b_{k}\right]$ is a monic polynomial in $b_{k}$ which vanishes identically on $Z(n, l)$ and therefore also on $Z^{\prime}$, so $Z^{\prime}$ is finite over $Z(n, L)$. Thus $Z^{\prime}$ and $Z=Z(n, L)$ are as in Definition 4.8.1

Lemma 4.9.3. Let $Z^{\prime}=Z^{\prime}(r, k)$ be a special arrangement over $Z=Z(n, L)$, where $L=[l] \backslash\{k\}$. Then we have

$$
I_{m}\left(Z^{\prime}\right) \subseteq I_{n, L}(m, r, k-1),
$$

where $I_{n, L}(m, r, k-1) \subseteq R(n, L)$ is the ideal of $Y_{n, L}(m, r, k-1)$.

Proof. Let $\pi: Z^{\prime} \rightarrow Z(n, L)$ be the projection. If $P$ is a point of $Z(n, L)$ such that the fiber $\pi^{-1}(P)$ has at least $m$ points, then, in the notation of Definitions 4.8.1 and 4.8.2 we have $\mu_{P}(S) \geq m$.

By Lemmas 4.8 .3 and 4.9 .2 it follows that $Y_{n, L}(m, r, k-1) \subseteq V_{m}\left(Z^{\prime}\right)$. Since $Y_{n, L}(m, r, k-1)$ is reduced, this implies $I_{m}\left(Z^{\prime}\right) \subseteq I_{n, L}(m, r, k-1)$.

Lemma 4.9.4. For $n=2$, with the notation of Lemma 4.9.3, we have equality in (125).

Proof. By Lemma 4.9.3 we have $I_{m}\left(Z^{\prime}\right) \subseteq I(m, r, k-1)$ in $R(2, L)$. This containment implies equality trivially if $I_{m}\left(Z^{\prime}\right)=(1)$ or $I(m, r, k-1)=0$. For $m=0$ we have $I(m, r, k-1)=0$. For $r=0, Z^{\prime}$ is empty, so $I_{m}\left(Z^{\prime}\right)=(1)$ for $m>0$. Hence we may assume $m, r>0$.

For $m=1$, the definition yields that $I_{1}\left(Z^{\prime}\right)$ is the kernel of the ring homomorphism $R \rightarrow S$ corresponding to the projection $\pi: Z^{\prime} \rightarrow Z$, that is, the ideal of the image of $Z^{\prime}$ in $Z$. The latter coincides with $Y(1, r, k-1)$ by construction.

Since $\left(b_{k}-y_{1}\right)\left(b_{k}-y_{2}\right)$ vanishes on $Z^{\prime}$, we have $I_{m}\left(Z^{\prime}\right)=(1)$ for $m>2$. If $r=1$, then $b_{k}-y_{1}$ vanishes on $Z^{\prime}$, and if $k>1$, then $b_{k}+b_{1}-y_{1}-y_{2}$ vanishes on $Z^{\prime}$. Hence we have $I_{m}\left(Z^{\prime}\right)=(1)$ for $r=1$ or $k>1$, and $m>1$. This leaves only the case $m=r=2, k=1$, in which we are to show

$$
I(2,2,0) \subseteq I_{2}\left(Z^{\prime}\right) .
$$

We have already seen (Corollary 4.6.3) that $I(2,2,0)=(\mathbf{x}, \mathbf{a})$. Thus we have only to show that $x_{1}, x_{2}$ and the $a_{i}$ for $i \in L=[l] \backslash\{1\}$ belong to $I_{2}\left(Z^{\prime}\right)$. For this we need only observe that $x_{1}, x_{2}$ and $a_{i}$ are the $b_{1}$ coefficients of the following polynomials, which vanish on $Z^{\prime}$ :

$$
\begin{gathered}
\left(b_{1}-y_{2}\right) x_{1}, \\
\left(b_{1}-y_{1}\right) x_{2}, \\
\left(b_{1}-y_{1}\right) a_{i}+\left(b_{i}-y_{2}\right) x_{1} .
\end{gathered}
$$

For the vanishing of the last of these, note that it is congruent modulo $\left(\left(b_{1}-y_{2}\right) x_{1}, a_{1}\right)$ to the determinant

$$
\operatorname{det}\left[\begin{array}{lll}
a_{i} & b_{i} & 1 \\
a_{1} & b_{1} & 1 \\
x_{1} & y_{1} & 1
\end{array}\right]
$$

from (96)), which vanishes on $Z(2, l)$. 
Lemma 4.9.5. For all $n$, with the notation of Lemma 4.9.3, we have equality in (125) locally on $U_{2}$, that is,

$$
I_{m}\left(Z^{\prime}\right)_{P}=I_{n, L}(m, r, k-1)_{P}
$$

for all $P \in U_{2} \cap Z(n, L)$.

Proof. First consider a point $P \in U_{1} \cap Z(n, L)$. By Lemma 4.4.3 $P$ lies on a unique component $W_{f}$, for some $f: L \rightarrow[n]$, and $Z(n, L)$ coincides locally with $Z_{1}=W_{f} \cong E^{n}$. Since $\pi: Z^{\prime} \rightarrow Z(n, L)$ maps $W_{g}$ into $W_{\left.g\right|_{L}}$, every point of $Z^{\prime}$ lying over $P$ belongs to a component $V\left(a_{k}\right) \cap W_{g}$ with $\left.g\right|_{L}=f$ and hence $g(k) \in T=[r] \backslash f([k-1])$. We can replace $Z^{\prime}$ with the union $Z_{1}^{\prime}$ of only these components, without changing $S_{P}$ and therefore without changing the local ideals $I_{m}\left(Z^{\prime}\right)_{P}$.

Identifying $Z_{1}$ with $E^{n}=\operatorname{Spec} k[\mathbf{x}, \mathbf{y}], Z_{1}^{\prime}$ is the union of subspaces

$$
V\left(x_{j}, b_{k}-y_{j}\right) \subseteq \operatorname{Spec} k\left[\mathbf{x}, \mathbf{y}, b_{k}\right]
$$

over all $j \in T$. Thus the defining ideal $J_{1}$ of $Z_{1}^{\prime}$ contains

$$
J=\prod_{j \in T}\left(x_{j}, b_{k}-y_{j}\right) .
$$

From this we see that $I_{m}\left(Z_{1}^{\prime}\right)$ contains all square-free monomials of degree $|T|+1-m$ in the variables $\left\{x_{j}: j \in T\right\}$. These monomials generate the ideal of the union of coordinate subspaces

$$
\bigcup V\left(x_{j}: j \in T_{1}\right) \cap Z_{1}, \quad \text { over subsets } T_{1} \subseteq T \text { of size }\left|T_{1}\right| \geq m .
$$

This union coincides locally with $Y_{n, L}(m, r, k-1)$ at $P$, so we have shown that $I_{n, L}(m, r, k-1)_{P} \subseteq I_{m}\left(Z^{\prime}\right)_{P}$. We already have the reverse containment by Lemma 4.9 .3 .

For $P \in U_{2} \backslash U_{1}$, the local picture of $Z(n, L)$ is given by Lemma 4.4.7 as $E^{[n] \backslash N} \times$ $Z\left(N, L^{\prime}\right)$ for some $N=\{p, q\} \subseteq[n]$, and $L^{\prime} \subseteq L$. Let $F$ be the equivalence class of functions $f: L \rightarrow[n]$ defined in Lemma 4.4.7, containing all $f$ such that $P \in W_{f}$. We put $Z_{1}=E^{[n] \backslash N}$ and $Z_{2}=Z\left(N, L^{\prime}\right)$.

As before, $Z^{\prime}$ coincides locally over $P$ with the union of its components $V\left(a_{k}\right) \cap$ $W_{g}$, where $\left.g\right|_{L}=f$ for some $f \in F$. We divide these into two classes. The first consists of components with $g(k) \notin N$. These have a point lying over $P$ only if $g(k)$ belongs to

$$
T=([r] \backslash N) \backslash f([k-1]) .
$$

Note that $T$ is well defined, since $f([k-1]) \cup N$ depends only on $F$. The union of these components is $Z_{1}^{\prime} \times Z_{2}$, where $Z_{1}^{\prime} \subseteq \mathbb{A}^{1} \times Z_{1}$ is the union of the subspaces $V\left(x_{j}, b_{k}-y_{j}\right)$ over all $j \in T$. Let $R_{1}=\operatorname{Spec} k\left[\mathbf{x}_{[n] \backslash N}, \mathbf{y}_{[n] \backslash N}\right]$ be the coordinate ring of $Z_{1}$. Repeating our analysis of the case $P \in U_{1}$, we see that for each $s$, $I_{s}\left(Z_{1}^{\prime}\right)$ contains the ideal $I_{s}^{\prime} \subseteq R_{1}$ generated by all square-free monomials of degree $|T|+1-s$ in the variables $\left\{x_{j}: j \in T\right\}$.

The second class consists of components $V\left(a_{k}\right) \cap W_{g}$ of $Z^{\prime}$ with $\left.g\right|_{L} \in F$ and $g(k) \in N$. The union of these components is $Z_{1} \times Z_{2}^{\prime}$, where $Z_{2}^{\prime}$ is the special arrangement $Z^{\prime}\left(r^{\prime}, k^{\prime}\right)$ over $Z_{2}$, with extra coordinate $b_{k}$, and $r^{\prime}=|N \cap[r]|, k^{\prime}-1=$ $\left|L^{\prime} \cap[k-1]\right|$. By Lemma 4.9.4 we have $I_{N, L^{\prime}}\left(t, r^{\prime}, k^{\prime}-1\right)=I_{t}\left(Z_{2}^{\prime}\right)$ for all $t$. Note that this is still correct when $r^{\prime}=0$ and $Z_{2}^{\prime}$ is empty. 
Using Lemmas 4.8.4 and 4.8.5 we see that for $s+t=m+1$ we have $I_{s}^{\prime} \otimes$ $I_{N, L^{\prime}}\left(t, r^{\prime}, k^{\prime}-1\right) \subseteq I_{m}\left(Z^{\prime}\right)$. By Lemma 4.6.4, the ideal

$$
I=\sum_{s+t=m+1} I_{s}^{\prime} \otimes I_{N, L^{\prime}}\left(t, r^{\prime}, k^{\prime}-1\right) \subseteq I_{m}\left(Z^{\prime}\right)
$$

is equal to its radical. Here we implicitly used the identity

$$
I_{s}^{\prime} \otimes I_{N, L^{\prime}}\left(t, r^{\prime}, k^{\prime}-1\right)=\left(I_{s}^{\prime} \otimes R\left(N, L^{\prime}\right)\right) \cap\left(R_{1} \otimes I_{N, L^{\prime}}\left(t, r^{\prime}, k^{\prime}-1\right)\right),
$$

which holds because $R_{1} / I_{s}^{\prime}$ and $R\left(N, L^{\prime}\right) / I_{N, L^{\prime}}\left(t, r^{\prime}, k^{\prime}-1\right)$ are flat over the field $k$. We have

$$
\begin{aligned}
V(I) & =\bigcap_{s+t=m+1}\left(V\left(I_{s}^{\prime}\right) \times Z_{2}\right) \cup\left(Z_{1} \times Y_{N, L^{\prime}}\left(t, r^{\prime}, k^{\prime}-1\right)\right) \\
& =\bigcup_{s+t=m} V\left(I_{s}^{\prime}\right) \times Y_{N, L^{\prime}}\left(t, r^{\prime}, k^{\prime}-1\right) .
\end{aligned}
$$

The second equality here follows because the closed subsets $V\left(I_{s}^{\prime}\right) \subseteq Z_{1}$ and $Y_{N, L^{\prime}}\left(t, r^{\prime}, k^{\prime}-1\right) \subseteq Z_{2}$ decrease as $s$ and $t$ increase. Unraveling the local isomorphism of $Z(n, L)$ with $Z_{1} \times Z_{2}$ we see that the union in (136) coincides locally at $P$ with $Y_{n, L}(m, r, k-1)$. This shows that $I_{n, L}(m, r, k-1)_{P} \subseteq I_{m}\left(Z^{\prime}\right)_{P}$, and we already have the reverse containment by Lemma 4.9.3, as before.

The next three lemmas contain specific consequences of the preceding results that we will use in 4.10. Throughout, the special arrangements are over $Z=Z(n, L)$, where $L=[l] \backslash\{k\}$, and all ideals $I\left(m^{\prime}, r^{\prime}, k^{\prime}\right)$ considered are in the ring $R(n, L)$. For readability, we suppress the subscripts, writing $I\left(m^{\prime}, r^{\prime}, k^{\prime}\right)$ for $I_{n, L}\left(m^{\prime}, r^{\prime}, k^{\prime}\right)$ and $Y\left(m^{\prime}, r^{\prime}, k^{\prime}\right)$ for $Y_{n, L}\left(m^{\prime}, r^{\prime}, k^{\prime}\right)$.

Lemma 4.9.6. Let $Z^{\prime}=Z^{\prime}(r, k)$ be a special arrangement over $Z=Z(n, L)$, where $L=[l] \backslash\{k\}$. Assume that the coordinate ring $R(n, L)$ is a free $k[\mathbf{y}]$-module with basis $B$ such that each ideal $I\left(m^{\prime}, r^{\prime}, k^{\prime}\right) \subseteq R(n, L)$ is spanned by a subset of $B$. In particular, for $r^{\prime}=r, k^{\prime}=k-1$, we have $B=\bigcup_{j=0}^{r} B_{j}$, where the $B_{j}$ are disjoint, such that $I(m, r, k-1)=k[\mathbf{y}] \cdot \bigcup_{j<m} B_{j}$ for all $m$. Then the coordinate ring $S=\mathcal{O}\left(Z^{\prime}\right)$ is a free $k[\mathbf{y}]$-module with basis

$$
B^{\prime}=\bigcup_{m=1}^{r} B_{m} \cdot\left\{1, b_{k}, \ldots, b_{k}^{m-1}\right\}
$$

Moreover, for all $m^{\prime}, r^{\prime}, k^{\prime}$, if $A \subseteq B$ spans $I\left(m^{\prime}, r^{\prime}, k^{\prime}\right)$, then $A^{\prime}=A \cdot\left\{1, b_{k}, b_{k}^{2}, \ldots\right\}$ $\cap B^{\prime}$ spans $I\left(m^{\prime}, r^{\prime}, k^{\prime}\right) S$.

Proof. By Lemma 4.5.2, the coordinate ring $S$ of $Z^{\prime}$ is a torsion-free $k[\mathbf{y}]$-module. By Lemma 4.9.5 we have $I_{m}\left(Z^{\prime}\right)_{P}=I(m, r, k-1)_{P}$ for all $m$ and all $P \in U_{2}$. Thus the hypotheses of Lemma 4.8.6 are satisfied and we have (137) immediately. For Lemma 4.8.6 to also yield the last conclusion we need to have $I_{m}\left(Y^{\prime}\right)_{P}=$ $I\left(m^{\prime}, r^{\prime}, k^{\prime}\right)_{P}+I(m, r, k-1)_{P}$ for $P \in U_{1}$ and $m>0$, where $Y^{\prime}$ is the schemetheoretic preimage $\pi^{-1} Y\left(m^{\prime}, r^{\prime}, k^{\prime}\right)$ in $Z^{\prime}$, that is, $Y^{\prime}=V\left(J^{\prime}\right)$ where $J^{\prime}=$ $I\left(m^{\prime}, r^{\prime}, k^{\prime}\right) S$.

As in the proof of Lemma 4.9.2, if $P$ is a point of $U_{1} \cap Z(n, L)$ belonging to the unique component $W_{f}$, then $Z^{\prime}$ contains $|T \cap[r] \backslash f([k-1])|$ distinct points lying over $P$, where $T=\left\{j: P \in V\left(x_{j}\right)\right\}$. If $P$ belongs to $U_{1} \cap Y(m, r, k-1)$, then (since $W_{f}$ is unique) we have $|T \cap[r] \backslash f([k-1])| \geq m$. If $P$ also belongs to $Y\left(m^{\prime}, r^{\prime}, k^{\prime}\right)$, then the points lying over $P$ belong to $Y^{\prime}$, which shows that $V_{m}\left(Y_{\text {red }}^{\prime}\right) \cap U_{1} \supseteq Y\left(m^{\prime}, r^{\prime}, k^{\prime}\right) \cap$ 
$Y(m, r, k-1) \cap U_{1}$ as sets. By Corollary 4.4.5 the last intersection is schemetheoretically reduced, so we have $I_{m}\left(Y_{\text {red }}^{\prime}\right)_{P} \subseteq I\left(m^{\prime}, r^{\prime}, k^{\prime}\right)_{P}+I(m, r, k-1)_{P}$ for all $P \in U_{1}$.

Let $J \subseteq R(n, L)\left[b_{k}\right]$ be the defining ideal of $Z^{\prime}$, so $S=R(n, L)\left[b_{k}\right] / J$. We defined $J^{\prime}$ as an ideal in $S$, but we can also regard it as an ideal in $R(n, L)\left[b_{k}\right]$ which contains $J$. Obviously $J^{\prime}$ is contained in the defining ideal $\sqrt{ } J^{\prime}$ of $Y_{\text {red }}^{\prime}$. This implies $I_{m}\left(Y^{\prime}\right) \subseteq I_{m}\left(Y_{\text {red }}^{\prime}\right)$ and hence $I_{m}\left(Y^{\prime}\right)_{P} \subseteq I\left(m^{\prime}, r^{\prime}, k^{\prime}\right)_{P}+I(m, r, k-1)_{P}$. For the reverse containment, we clearly have $I_{m}\left(Y^{\prime}\right) \supseteq I_{1}\left(Y^{\prime}\right)=J^{\prime} \cap R(n, L) \supseteq I\left(m^{\prime}, r^{\prime}, k^{\prime}\right)$ for $m>0$, and we have $I_{m}\left(Y^{\prime}\right)_{P} \supseteq I(m, r, k-1)_{P}$ because $I(m, r, k-1)_{P}=$ $I_{m}\left(Z^{\prime}\right)_{P}$ and $J^{\prime}$ contains $J$.

Lemma 4.9.7. Under the hypotheses of Lemma 4.9.6, each ideal $I\left(m^{\prime}, r^{\prime}, k^{\prime}\right) S$ is equal to its radical, so $S / I\left(m^{\prime}, r^{\prime}, k^{\prime}\right) S$ is the coordinate ring of the reduced preimage $\pi^{-1} Y\left(m^{\prime}, r^{\prime}, k^{\prime}\right)$ in $Z^{\prime}$, and this coordinate ring is a free $k[\mathbf{y}]$-module.

Proof. Lemma 4.9.6 implies that $S / I\left(m^{\prime}, r^{\prime}, k^{\prime}\right) S$ is a free $k[\mathbf{y}]$-module and therefore a subring of $k(\mathbf{y}) \otimes S / I\left(m^{\prime}, r^{\prime}, k^{\prime}\right) S$. Let $J$ be the ideal of $Z^{\prime}$ as a subscheme of $Z(n, l)$, so $S \cong R(n, l) / J$. Both $J$ and $\sqrt{ } I\left(m^{\prime}, r^{\prime}, k^{\prime}\right) R(n, l)$ belong to the lattice in Lemma 4.4.4 Moreover $I\left(m^{\prime}, r^{\prime}, k^{\prime}\right) R(n, l)_{P}=\sqrt{ } I\left(m^{\prime}, r^{\prime}, k^{\prime}\right) R(n, l)_{P}$ for all $P \in U_{1}$, as follows easily from Lemma 4.4.3. Hence Lemma 4.4.4 implies that $I\left(m^{\prime}, r^{\prime}, k^{\prime}\right) R(n, l)_{P}+J_{P}$ is equal to its radical for all $P \in U_{1}$, and by Lemma 4.4.2 this implies that $\left(S / I\left(m^{\prime}, r^{\prime}, k^{\prime}\right) S\right)_{Q}$ is a reduced ring for all $Q \in \hat{U}_{1}$. In particular, taking $Q=0, k(\mathbf{y}) \otimes S / I\left(m^{\prime}, r^{\prime}, k^{\prime}\right) S$ is a reduced ring.

Lemma 4.9.8. Let $Z^{\prime}=Z^{\prime}(r, k)$ and $Z^{\prime \prime}=Z^{\prime}(r-1, k)$ be special arrangements over $Z=Z(n, L)$, where $L=[l] \backslash k$. Let $Z^{\prime}=\operatorname{Spec} S$, and let $J^{\prime} \subseteq S$ be the ideal of $Z^{\prime \prime}$ as a closed subscheme of $Z^{\prime}$. Assume that $R(n, L)$ is a free $k[\mathbf{y}]$-module with a basis $B$ such that each ideal $I\left(m^{\prime}, r^{\prime}, k^{\prime}\right)$ is spanned by a subset of $B$. Then $J^{\prime}$ is a free $k[\mathbf{y}]$-module with a basis $B^{\prime}$ such that every ideal $I\left(m^{\prime}, r^{\prime}, k^{\prime}\right) S \cap J^{\prime}$ is spanned by a subset of $B^{\prime}$.

Proof. For each basis element $p \in B$, let $m(p)$ be the unique nonnegative integer $m$ such that $p$ belongs to $I(m+1, r, k-1)$ but not to $I(m, r, k-1)$. Similarly, let $m^{\prime}(p)$ be the unique $m^{\prime}$ such that $p$ belongs to $I\left(m^{\prime}+1, r-1, k-1\right)$ but not to $I\left(m^{\prime}, r-1, k-1\right)$. Thus by Lemma 4.9.6 the set of elements

$$
B_{1}=\left\{p b_{k}^{j}: p \in B, j<m(p)\right\}
$$

is a free $k[\mathbf{y}]$-module basis of $S$ and the set

$$
B_{1}^{\prime}=\left\{p b_{k}^{j}: p \in B, j<m^{\prime}(p)\right\}
$$

is a free $k[\mathbf{y}]$-module basis of $S / J^{\prime}$. From the definition we have

$$
Y(m+1, r-1, k-1) \subseteq Y(m+1, r, k-1) \subseteq Y(m, r-1, k-1),
$$

hence

$$
I(m, r-1, k-1) \subseteq I(m+1, r, k-1) \subseteq I(m+1, r-1, k-1) .
$$

Therefore we have $m^{\prime}(p) \leq m(p) \leq m^{\prime}(p)+1$ for all $p \in B$.

For each $p \in B$ such that $m(p)=m^{\prime}(p)+1$, the element $p_{0}=p b_{k}^{m(p)-1}$ belongs to $B_{1}$ but not to $B_{1}^{\prime}$. There is, however, a unique $k[\mathbf{y}]$-linear combination $p_{1}$ of the elements in $B_{1}^{\prime}$ such that $p_{0} \equiv p_{1}\left(\bmod J^{\prime}\right)$. Set $q=p_{0}-p_{1}$, so we have $q \in J^{\prime}$, and $q$ is congruent to $p_{0}$ modulo $k[\mathbf{y}] B_{1}^{\prime}$. Let $B^{\prime}$ be the set of all such elements $q$. 
The union $B^{\prime} \cup B_{1}^{\prime}$ is again a basis of $S$, while $B_{1}^{\prime}$ is a basis of $S / J^{\prime}$ and $B^{\prime}$ is contained in $J^{\prime}$. This implies that $B^{\prime}$ spans $J^{\prime}$, that is, $J^{\prime}$ is a free $k[\mathbf{y}]$-module with basis $B^{\prime}$.

By Lemma 4.9.6, $I\left(m^{\prime}, r^{\prime}, k^{\prime}\right) S$ is spanned by the subset

$$
A_{1}=\left\{p b_{k}^{j} \in B_{1}: p \in I\left(m^{\prime}, r^{\prime}, k^{\prime}\right)\right\} .
$$

Consider an element $p \in I\left(m^{\prime}, r^{\prime}, k^{\prime}\right) \cap B$ with $p_{0}=p b_{k}^{m(p)-1} \in B_{1} \backslash B_{1}^{\prime}$, and the corresponding element $q$ constructed as above. By Lemma 4.9.6 applied to $Z^{\prime \prime}$, the set $A_{1}^{\prime}=\left\{p^{\prime} b_{k}^{j} \in B_{1}^{\prime}: p^{\prime} \in I\left(m^{\prime}, r^{\prime}, k^{\prime}\right)\right\}$ spans $I\left(m^{\prime}, r^{\prime}, k^{\prime}\right) S /\left(I\left(m^{\prime}, r^{\prime}, k^{\prime}\right) S \cap J^{\prime}\right)$. Hence there is a $k[\mathbf{y}]$-linear combination of the elements of $A_{1}^{\prime}$ congruent to $p_{0}$ modulo $I\left(m^{\prime}, r^{\prime}, k^{\prime}\right) S \cap J^{\prime}$. By uniqueness this linear combination must be $p_{1}$, so $q$ belongs to $I\left(m^{\prime}, r^{\prime}, k^{\prime}\right) S \cap J^{\prime}$. Let $A^{\prime} \subseteq B^{\prime}$ be the set of these elements $q$. As before, $A^{\prime} \cup A_{1}^{\prime}$ spans $I\left(m^{\prime}, r^{\prime}, k^{\prime}\right) S$, while $A_{1}^{\prime}$ is a basis of $I\left(m^{\prime}, r^{\prime}, k^{\prime}\right) S /\left(I\left(m^{\prime}, r^{\prime}, k^{\prime}\right) S \cap J^{\prime}\right)$ and $A^{\prime}$ is contained in $I\left(m^{\prime}, r^{\prime}, k^{\prime}\right) S \cap J^{\prime}$. This implies that $A^{\prime}$ spans $I\left(m^{\prime}, r^{\prime}, k^{\prime}\right) S \cap$ $J^{\prime}$.

4.10. Basis construction. We are finally ready to establish the lemmas that will allow us to construct the basis required by Theorem 4.2. As explained in $\$ 4.3$, the construction proceeds by induction, in three stages. In the first stage we construct a basis of $R(n, l) / I(1,1, l)$ from a basis of $R(n-1, l)$. In the second stage we construct a basis of $R(n, l) / I(1,1, t-1)$ from a basis of $R(n, l) / I(1,1, t)$ for $1 \leq t \leq l$. In the third and final stage we construct a basis of $R(n, l)$ from a basis of $R(n, l) / I(1,1,0)$. The three stages are given by corresponding lemmas below: the first stage by Lemma 4.10.2, the third stage by Lemma 4.10.5 and the second stage by Lemma 4.10.7. In the order we present them, each lemma requires progressively more algebraic and geometric machinery to prove; this is why we consider the third stage before the second. All stages of the construction involve the rings $R(n, l) / I(1,1, t)$. Before we give any of the basis construction lemmas, we first need to define the type of basis we want in the rings $R(n, l) / I(1,1, t)$ as well as $R(n, l)$.

Definition 4.10.1. A common ideal basis of $R(n, l)$ is a set $B$ for which the conclusion of Theorem 4.2 holds: namely, $R(n, l)$ is a free $k[\mathbf{y}]$-module with basis $B$ and every ideal $I(m, r, k)$ is spanned by a subset of $B$. Given $t \in\{0\} \cup[l]$, let $J=I(1,1, t)$. A common ideal basis of $R(n, l) / J$ is a set $B$ such that $R(n, l) / J$ is a free $k[\mathbf{y}]$-module with basis $B$ and every ideal

$$
\sqrt{ }(I(m, r, k)+J) / J
$$

is spanned as a $k[\mathbf{y}]$-module by a subset of $B$.

For the sake of clarity let us point out that if a common ideal basis of $R(n, l)$ exists, then $R(n, l) /(I(m, r, k)+J)$ is a free $k[\mathbf{y}]$-module and hence a subring of $k(\mathbf{y}) \otimes R(n, l) /(I(m, r, k)+J)$. By Corollary 4.4.5, the latter ring is reduced, so $I(m, r, k)+J=\sqrt{ }(I(m, r, k)+J)$. In light of Theorem 4.2, the radical sign in (143) is therefore superfluous. However, in the construction of our basis in stages, we will have to construct common ideal bases for the rings $R(n, l) / J$ first, before we reach $R(n, l)$ itself. For this reason we have taken care to define a common ideal basis of $R(n, l) / J=\mathcal{O}(Y(1,1, t))$ to be a common basis of the ideals of the reduced arrangements $Y(m, r, k) \cap Y(1,1, t)$, without assuming in advance that these intersections are scheme-theoretically reduced. 
Each stage of our basis construction procedure involves the action of the circular shift permutation $\theta=\left(\begin{array}{llll}1 & 2 & \ldots & n\end{array}\right)$ which appeared earlier in Lemma 4.7.3 Let us briefly note what its action means in various contexts. Geometrically, $\theta$ acts on $E^{n}$ as the right circular shift

$$
\theta\left(P_{1}, \ldots, P_{n}\right)=\left(P_{n}, P_{1}, \ldots, P_{n-1}\right),
$$

and on $E^{l}$ as the identity. In coordinates, $\theta$ acts by

$$
\theta x_{i}=x_{i+1}, \theta y_{i}=y_{i+1}(i<n), \quad \theta x_{n}=x_{1}, \theta y_{n}=y_{1},
$$

and fixes the coordinates $\mathbf{a}, \mathbf{b}$. The shift $\theta$ also acts on various indexing data, e.g., for subsets $S \subseteq[n]$ we have $\theta S=\theta(S)$, and for functions $f:[l] \rightarrow[n]$ we have $\theta f=\theta \circ f$. The various actions are mutually consistent: we have $\theta W_{f}=W_{\theta f}$, for example, and the ideal of $\theta Y(m, r, k)$ is $\theta I(m, r, k)$. The ring homomorphism defined by (145) corresponds to the inverse of the morphism $\theta$ given in (144). However, it is this definition that makes the notation consistent, as the reader can verify. In general, whenever a group $G$ acts on a scheme $X=\operatorname{Spec} R$, we must define $g \in G$ to act on $R$ by the ring homomorphism corresponding to the morphism $g^{-1}$ _ otherwise the left action of $G$ on $X$ would become a right action on $R$. In our situation, $\theta$ is merely a specially chosen element of the symmetric group $S_{n}$, which acts on $E^{n}$ by permuting the factors.

We turn now to the basis construction lemmas, beginning with the easiest. Throughout we will maintain the hypothesis that the bases we construct consist of homogeneous polynomials. Strictly speaking, we only need to maintain homogeneity with respect to the $x$-degree, although it is not hard to see on closer examination that everything in the construction also maintains homogeneity with respect to the $y$-degree.

Lemma 4.10.2. Suppose that $R(n-1, l)$ has a homogeneous common ideal basis. Then so does $R(n, l) / I(1,1, l)$.

Proof. We can assume we are given a common ideal basis of $R(n-1, l)$, represented by a set of homogeneous polynomials $B^{\prime} \subseteq k[\mathbf{x}, \mathbf{y}, \mathbf{a}, \mathbf{b}]$ not involving the variables $x_{n}, y_{n}$. We will show that $B=\theta B^{\prime}$ is the required basis of $R(n, l) / I(1,1, l)$.

Let $J=I(1,1, l)$. From the definition it is immediate that $Y(1,1, l) \cong Z(N, l) \times$ Spec $k\left[y_{1}\right]$, where $N=[n] \backslash\{1\}=\theta[n-1]$. This implies that $R(n, l) / J$ is a free $k[\mathbf{y}]$-module with basis $B$. It follows from Lemma 4.7.2 that for $r>0$, the image of $Y(m, r, k) \cap Y(1,1, l)$ under the isomorphism is $Y_{N, l}(m-1, r-1, k) \times \operatorname{Spec} k\left[y_{1}\right]$, so $\sqrt{ }(I(m, r, k)+J) / J=I_{N, l}(m-1, r-1, k) \otimes k\left[y_{1}\right]$. This shows that $B$ is a common ideal basis as far as the ideals $\sqrt{ }(I(m, r, k)+J) / J$ with $r>0$ are concerned, and those with $r=0$ are trivial.

The remaining stages in the basis construction procedure make use of the following technical lemma.

Lemma 4.10.3. Let $R$ be a $k[\mathbf{y}]$-algebra and let $J, I_{1}, \ldots, I_{m}$ be ideals of $R$ such that $R / I_{i}$ is a torsion-free $k[\mathbf{y}]$-module for all $i$, and for every intersection $I$ of some of the ideals $I_{i}$, we have $(I+J)_{P}=\sqrt{ }(I+J)_{P}$ for all $P \in U_{2}$. Assume that $J$ is a free $k[\mathbf{y}]$-module with basis $B^{\prime \prime}$ such that each ideal $I_{i} \cap J$ is spanned by a subset of $B^{\prime \prime}$, and that $R / J$ is a free $k[\mathbf{y}]$-module with basis $B^{\prime}$ such that each ideal $\sqrt{ }\left(I_{i}+J\right) / J$ is spanned by a subset of $B^{\prime}$. Then $R$ is a free $k[\mathbf{y}]$-module with a basis $B$ such that $J$ and all the ideals $I_{i}$ are spanned by subsets of $B$. 
Proof. Choosing an arbitrary representative $q \in R$ of each $J$-coset belonging to $B^{\prime}$, we can assume that $B^{\prime}$ is given as a subset of $R$. Then $R$ is a free $k[\mathbf{y}]$-module with basis $B^{\prime} \cup B^{\prime \prime}$. This basis need not be a common basis of the ideals $I_{i}$; to construct $B$ we have to modify it.

Given $q \in B^{\prime}$, let $\alpha$ be the set of indices $i$ for which $\sqrt{ }\left(I_{i}+J\right)$ contains $q$, and let $I=\bigcap_{i \in \alpha} I_{i}$. Then $\sqrt{ }(I+J)=\bigcap_{i \in \alpha} \sqrt{ }\left(I_{i}+J\right)$ contains $q$. For $Q \in \hat{U}_{2}$ we have $(I+J)_{Q}=\sqrt{ }(I+J)_{Q}$ by Lemma 4.4.2, hence $q \in(I+J)_{Q}$. Thus there exists an element $q_{1} \in R_{Q}$ satisfying

$$
q_{1} \in I_{Q}, \quad q_{1}-q \in J_{Q} .
$$

These conditions determine $q_{1}$ and $q_{1}-q$ uniquely modulo $(I \cap J)_{Q}$. Now, it follows from the hypotheses of the lemma that if $C \subseteq B^{\prime \prime}$ is the subset consisting of elements not belonging to $I \cap J$, then $J / I \cap J$ is a free $k[\mathbf{y}]$-module with basis $C$. Expanding the unique element $q_{1}-q \in J_{Q} /(I \cap J)_{Q}$ satisfying (146) in terms of the basis $C$, we have

$$
q_{1}=q+\sum_{\gamma} p_{\gamma} b_{\gamma}
$$

with $p_{\gamma} \in k[\mathbf{y}]_{Q}$ and $b_{\gamma} \in C$. The coefficients $p_{\gamma}$ are uniquely determined by (146) and (147), and the solution for any $Q$ is also the solution for $Q=0$. As in the proof of Lemma 4.4.9, it follows that as elements of $k(\mathbf{y})$, the coefficients $p_{\gamma} \in k[\mathbf{y}]_{Q}$ do not depend on $Q$ and hence belong to $k[\mathbf{y}]$. In particular, the polynomial $q_{1}$ defined in (147) is an element of $R$ satisfying (146) for all $Q \in \hat{U}_{2}$. The hypothesis that $R / I_{i}$ is a torsion-free $k[\mathbf{y}]$-module means that the canonical map $R / I_{i} \rightarrow k(\mathbf{y}) \otimes\left(R / I_{i}\right)$ is injective. Since $k(\mathbf{y}) \otimes\left(R / I_{i}\right)=\left(R / I_{i}\right)_{Q}$ for $Q=0$, and we have $q_{1} \in I_{(0)} \subseteq\left(I_{i}\right)_{(0)}$, this implies $q_{1} \in I_{i}$ for all $i \in \alpha$.

We now define $B$ to be the union of $B^{\prime \prime}$ and the set of elements $q_{1}$ constructed as above, for all $q \in B^{\prime}$. By (147) we have $q_{1} \equiv q(\bmod J)$. This implies that the elements $q_{1}$ form a basis of $R / J$, so $B$ is a basis of $R$.

For each $i$, the elements $q \in B^{\prime} \cap \sqrt{ }\left(I_{i}+J\right)$ span the $k[\mathbf{y}]$-module $\sqrt{ }\left(I_{i}+J\right) / J$ by hypothesis, and therefore span the vector space $k(\mathbf{y}) \otimes \sqrt{ }\left(I_{i}+J\right) / J=k(\mathbf{y}) \otimes$ $\left(\left(I_{i}+J\right) / J\right)$. Since the corresponding elements $q_{1}$ belong to $I_{i}$ and satisfy $q_{1} \equiv q$ $(\bmod J)$ ), it follows from the canonical isomorphism $I_{i} /\left(I_{i} \cap J\right) \rightarrow\left(I_{i}+J\right) / J$ that they span $k(\mathbf{y}) \otimes\left(I_{i} /\left(I_{i} \cap J\right)\right)$. By hypothesis the elements of $B^{\prime \prime} \cap I_{i}$ span $I_{i} \cap J$, so the elements of $B \cap I_{i}$ all together span $k(\mathbf{y}) \otimes I_{i}$. By Lemma 4.4.11, this implies they $\operatorname{span} I_{i}$ as a $k[\mathbf{y}]$-module.

To maintain homogeneity, we need a graded version of the preceding lemma. For the stage going from $R(n, l) / I(1,1,0)$ to $R(n, l)$ in our basis construction procedure we will want a bit more: the graded version should hold in each degree separately. To avoid cluttering the notation, we did not mention the grading in the proof of Lemma 4.10.3 but everything we need is already implicit there. To see this, consider the situation in which $R=\bigoplus_{d} R_{d}$ is a graded $k[\mathbf{y}]$-algebra with $k[\mathbf{y}] \subseteq R_{0}$, the ideals $I_{i}$ and $J$ are homogeneous, and the elements of $B^{\prime}$ and $B^{\prime \prime}$ are also homogeneous. Then the construction of the basis $B$ in the proof of the lemma can be carried out in each degree separately. More precisely, the proof yields a common basis for the homogeneous components of the ideals $J$ and $I_{i}$ in a given degree $d$, assuming only that we have bases $B_{d}^{\prime \prime}$ of $J_{d}$ and $B_{d}^{\prime}$ of $(R / J)_{d}$ satisfying 
the hypotheses of the lemma in degree $d$. Thus we have the following corollary to the proof of Lemma 4.10.3.

Corollary 4.10.4. Let $R, J$ and $I_{1}, \ldots, I_{m}$ be as in Lemma 4.10.3, but assume further that $R=\bigoplus_{d} R_{d}$ is graded, with $k[\mathbf{y}] \subseteq R_{0}$, and the ideals $J, I_{1}, \ldots, I_{m}$ are homogeneous. For a given degree $d$, suppose that $J_{d}$ is a free $k[\mathbf{y}]$-module with basis $B_{d}^{\prime \prime}$ such that each $\left(I_{i} \cap J\right)_{d}$ is spanned by a subset of $B_{d}^{\prime \prime}$, and that $(R / J)_{d}$ is a free $k[\mathbf{y}]$-module with basis $B_{d}^{\prime}$ such that each $\left(\sqrt{ }\left(I_{i}+J\right) / J\right)_{d}$ is spanned by a subset of $B_{d}^{\prime}$. Then $R_{d}$ is a free $k[\mathbf{y}]$-module with a basis $B_{d}$ such that $J_{d}$ and all $\left(I_{i}\right)_{d}$ are spanned by subsets of $B_{d}$.

We remark that the torsion-freeness hypothesis and the local reducedness condition $(I+J)_{P}=\sqrt{ }(I+J)_{P}$ in Lemma 4.10.3 and Corollary 4.10.4 are automatically satisfied in the situations where we will apply them. The ideals $I_{i}$ will always be ideals of (reduced) subspace arrangements $Y(m, r, k)$ or $Y(m, r, k) \cap Y(1,1, t)$. By Lemmas 4.5.2 and 4.7.2 their coordinate rings $R / I_{i}$ are torsion-free $k[\mathbf{y}]$-modules. The ideal $J$ will always be $I(1,1, t)$ for some $t$, so it follows from Lemma 4.6.5 that $V(I+J)$ is locally reduced on $U_{2}$.

It follows a posteriori from the conclusion of Lemma4.10.3 that if the reducedness hypotheses $(I+J)_{P}=\sqrt{ }(I+J)_{P}$ hold locally on $U_{2}$, we must actually have $(I+J)=$ $\sqrt{ }(I+J)$. This is similar to the situation we encountered earlier with Lemma 4.8.6 As in that case, we proved the lemma with the weaker local hypotheses so that we can verify them by reduction to the case $n=2$.

We come now to our second basis construction lemma, giving what is actually the third stage in the inductive process.

Lemma 4.10.5. Suppose that $R(n, l) / I(1,1,0)$ has a homogeneous common ideal basis. Then so does $R(n, l)$.

Proof. Let $J=I(1,1,0)$. By definition, $Y(1,1,0)=V\left(x_{1}\right)$, so $J=\sqrt{ }\left(x_{1}\right)$. By Lemma 4.6.6, $\sqrt{ }\left(x_{1}\right)=\left(x_{1}\right)$. Since $x_{1}$ does not vanish identically on any $W_{f}$, it is a non-zero-divisor in $R(n, l)$. Hence multiplication by $x_{1}$ is an isomorphism of $R(n, l)$ onto $J=\left(x_{1}\right)$.

We may assume we are given a homogeneous common ideal basis $B^{\prime}$ of $R(n, l) / J$. Suppose that in a given $x$-degree $d$, we can find a free $k[\mathbf{y}]$-module basis $B_{d}$ of $R(n, l)_{d}$ such that every $I(m, r, k)_{d}$ is spanned by a subset of $B_{d}$. We claim that $x_{1} \theta B_{d}$ is then a basis of $J_{d+1}$ with subsets spanning each $(I(m, r, k) \cap J)_{d+1}$. Granting this claim for the moment, we can apply Corollary 4.10 .4 with $B_{d+1}^{\prime \prime}=x_{1} \theta B_{d}$ and $B_{d+1}^{\prime}$ the degree $d+1$ part of the given basis $B^{\prime}$ to obtain a free $k[\mathbf{y}]$-module basis $B_{d+1}$ of $R(n, l)_{d+1}$ which is a common basis for every $I(m, r, k)_{d+1}$. In degree zero, we can take $B_{0}=B_{0}^{\prime}$, since $R(n, l)_{0}=(R(n, l) / J)_{0}$. We can then construct $B_{d}$ by induction for all degrees $d$, obtaining a common ideal basis $B=\bigcup_{d} B_{d}$ of $R(n, l)$.

It remains to prove the claim. If $B_{d}$ is a free module basis of $R(n, l)_{d}$, then so is $\theta B_{d}$, and therefore $x_{1} \theta B_{d}$ is a basis of $J_{d+1}$, since multiplication by $x_{1}$ is an isomorphism of $R(n, l)_{d}$ onto $J_{d+1}$. To complete the proof, observe that for any ideal $I \subseteq R(n, l)$ we have $I \cap J=I \cap\left(x_{1}\right)=x_{1}\left(I:\left(x_{1}\right)\right)$. If $I$ is radical, then so is $I:\left(x_{1}\right)$, and $V\left(I:\left(x_{1}\right)\right)$ is the union of those components of $V(I)$ on which $x_{1}$ does not vanish identically. Applying this to $I=I(m, r, k)$ for $r>0$, we get $I(m, r, k):\left(x_{1}\right)=\theta I(m, r-1, k)$, and hence $I(m, r, k) \cap J=x_{1} \theta I(m, r-1, k)$. For $r>0$, this shows that if $B_{d}$ has a subset spanning $I(m, r-1, k)_{d}$, then $x_{1} \theta B_{d}$ has 
a subset spanning $(I(m, r, k) \cap J)_{d+1}$. This suffices, since $I(m, r, k)$ is trivially equal to 0 or (1) for $r=0$.

The remaining and most subtle stage of the basis construction procedure is the one going from $R(n, l) / I(1,1, t)$ to $R(n, l) / I(1,1, t-1)$, for $t \in[l]$. For this stage we will also assume we have a common ideal basis of $R(n, l-1)$, and apply the basis lifting technique developed in $\S \$ 4.8$ and 4.9 , Recall that the basis lifting involves the special arrangements $Z^{\prime}(r, k)$. In order to make use of them we need a lemma relating the ideal of $Y(1,1, t)$ as a closed subscheme of $Y(1,1, t-1)$ to the ideal of $Z^{\prime \prime}$ as a closed subscheme of $Z^{\prime}$, for a suitable pair of special arrangements $Z^{\prime \prime} \subseteq Z^{\prime}$. Before stating the lemma we fix some notation. Let $n>1, l>0$ and $t \in[l]$ be given. Let $Z^{\prime}=Z^{\prime}(n, t)$ be the special arrangement with $r=n$ and $k=t$, and let $Z^{\prime \prime}=Z^{\prime}(n-1, t)$.

Note that $Z^{\prime \prime}$ is a subarrangement of $Z^{\prime}$. The components of $Z^{\prime}$ that do not belong to $Z^{\prime \prime}$ are the subspaces

$$
V\left(a_{t}\right) \cap W_{f}=V\left(x_{n}\right) \cap W_{f}
$$

for $f:[l] \rightarrow[n]$ such that $f(t)=n, f(i) \neq n$ for $i \in[t-1]$. Let $Z_{1}$ be the union of these components. Then we have

$$
Z^{\prime}=Z^{\prime \prime} \cup Z_{1},
$$

with $Z^{\prime \prime}$ and $Z_{1}$ having no component in common.

Similarly, $Y(1,1, t)$ is a subarrangement of $Y(1,1, t-1)$, and the components of $Y(1,1, t-1)$ not belonging to $Y(1,1, t)$ are the subspaces

$$
V\left(x_{1}\right) \cap W_{f}
$$

for $f:[l] \rightarrow[n]$ such that $f(t)=1, f(i) \neq 1$ for $i \in[t-1]$. Letting $Z_{0}$ be their union, we have as above

$$
Y(1,1, t-1)=Y(1,1, t) \cup Z_{0},
$$

with $Y(1,1, t)$ and $Z_{0}$ having no component in common. Observe that $Z_{0}=\theta Z_{1}$. The key fact about this set-up is as follows.

Lemma 4.10.6. With $n, l, t, Z^{\prime}, Z^{\prime \prime}$ and $Z_{0}$ as above, we have

$$
\theta Z^{\prime \prime} \cap Z_{0}=Y(1,1, t) \cap Z_{0},
$$

scheme-theoretically on $U_{2}$. In other words, for all $P \in U_{2}$ we have equality of ideals $\theta I\left(Z^{\prime \prime}\right)_{P}+I\left(Z_{0}\right)_{P}=I(1,1, t)_{P}+I\left(Z_{0}\right)_{P}$.

Proof. We caution the reader immediately that in general the intersections in (152) are not scheme-theoretically reduced, so it is not enough to check the result settheoretically. Instead, we must use our knowledge of the local picture on $U_{2}$ to write down equations. If $P$ is a point of the intersection on either side of (152), then we have $P \in W_{f} \cap W_{g}$ for some $f, g$ with $f(t) \neq 1, g(t)=1$, so $P$ is not in $U_{1}$. Thus we need only consider points $P \in U_{2} \backslash U_{1}$.

The schemes in question are subschemes of $Z(n, l)$. Fix a point $P \in U_{2} \backslash U_{1}$, and let $N, L, \sim, F$ and $h \in F$ be as in Lemma 4.4.7. We may replace $Z(n, l)$ with $E^{[n] \backslash N} \times Z(N, L)$ without changing any of the local ideals at $P$.

First consider the case $1 \notin N$. If $P \in Z_{0}$, we must have $h(t)=1$. But if $P \in \theta Z^{\prime \prime}$ or $P \in Y(1,1, t)$, we must have $h(t) \neq 1$. Hence both intersections in (152) are locally empty at $P$. 
For the case $1 \in N$ we may assume $h(t) \in N$, and hence $t \in L$, as otherwise $P \notin Z_{0}$ and the result is trivial. Under the local isomorphism of $Z(n, l)$ with $E^{[n] \backslash N} \times Z(N, L), Z_{0}$ coincides locally with $E^{[n] \backslash N} \times \tilde{Z}_{0}$, where $\tilde{Z}_{0} \subseteq Z(N, L)$ is the subspace arrangement

$$
\tilde{Z}_{0}=\bigcup_{f} V\left(x_{1}\right) \cap W_{f},
$$

over $f: L \rightarrow N$ such that $f(t)=1, f(i) \neq 1$ for $i \in[t-1] \cap L$.

Similarly, $Y(1,1, t)$ coincides locally with $E^{[n] \backslash N} \times Y_{N, L}(1,1, s)$, where $s=$ $|[t] \cap L|$, and $\theta Z^{\prime \prime}$ coincides with $E^{[n] \backslash N} \times \tilde{Z}$, where

$$
\tilde{Z}=\bigcup_{f} V\left(a_{t}\right) \cap W_{f},
$$

over $f: L \rightarrow N$ such that $f(t) \neq 1, f(i)=1$ for $i \in[t-1] \cap L$.

We are to show that $\tilde{Z} \cap \tilde{Z}_{0}=Y_{N, L}(1,1, s) \cap \tilde{Z}_{0}$ as subschemes of $Z(N, L)$. Renaming the indices so that $N$ becomes [2], $L$ becomes $[\tilde{l}]$ and (hence) $t$ becomes $s$, our subschemes become subschemes of $Z(2, \tilde{l})$ :

$$
\begin{aligned}
\tilde{Z}_{0}=\bigcup V\left(x_{1}\right) \cap W_{f}: & f(s)=1, f(i)=2 \text { for } i<s, \\
\tilde{Z}=\bigcup V\left(x_{2}\right) \cap W_{f}: & f(s)=2, f(i)=1 \text { for } i<s, \\
Y(1,1, s)=\bigcup V\left(x_{1}\right) \cap W_{f}: & f(s)=2, f(i)=2 \text { for } i<s .
\end{aligned}
$$

Let $I \subseteq R(2, \tilde{l})$ be given by

$$
I=\left(a_{s}-x_{1}, b_{s}-y_{1}\right)+\sum_{i<s}\left(a_{i}-x_{2}, b_{i}-y_{2}\right) .
$$

By Lemma 4.4.8, $I=\sqrt{ } I$ and $V(I) \cong Z(2, \tilde{L})$, where $\tilde{L}=[\tilde{l}] \backslash[s]$. By Corollary 4.6.3 or Lemma 4.6.6, we have $I(1,1,0)=\left(x_{1}\right)$ in $Z(2, \tilde{L})$, which shows that $\left(x_{1}\right)+I$ is a radical ideal. Since we clearly have $V\left(\left(x_{1}\right)+I\right)=\tilde{Z}_{0}$ set-theoretically, it follows that

$$
I\left(\tilde{Z}_{0}\right)=\left(x_{1}\right)+\left(a_{s}-x_{1}, b_{s}-y_{1}\right)+\sum_{i<s}\left(a_{i}-x_{2}, b_{i}-y_{2}\right) .
$$

By symmetry, we have

$$
I(\tilde{Z})=\left(x_{2}\right)+\left(a_{s}-x_{2}, b_{s}-y_{2}\right)+\sum_{i<s}\left(a_{i}-x_{1}, b_{i}-y_{1}\right),
$$

and by Corollary 4.6 .3 we have

$$
I(1,1, s)=\left(x_{1}\right)+\left(a_{s}-x_{2}, b_{s}-y_{2}\right)+\sum_{i<s}\left(a_{i}-x_{2}, b_{i}-y_{2}\right) .
$$

Now we see immediately that both $I(\tilde{Z})+I\left(\tilde{Z}_{0}\right)$ and $I(1,1, s)+I\left(\tilde{Z}_{0}\right)$ contain $\left(x_{1}-x_{2}, y_{1}-y_{2}\right)$ and hence they both reduce to

$$
\left(x_{1}, x_{2}, y_{1}-y_{2}\right)+\sum_{i \leq s}\left(a_{i}, b_{i}-y_{1}\right) .
$$


As was the case with Lemma 4.6.5, one can show using Theorem 4.2 that the conclusion of Lemma 4.10.6 actually holds everywhere, and not just on $U_{2}$. As before, the result on $U_{2}$ suffices for our purposes, and the restriction to $U_{2}$ enables us to prove it by reduction to the case $n=2$.

Lemma 4.10.7. Given $n>1, l>0$ and $t \in[l]$, suppose that $R(n, l-1)$ and $R(n, l) / I(1,1, t)$ each have a homogeneous common ideal basis. Then so does $R(n, l) / I(1,1, t-1)$.

Proof. To simplify notation let $R=R(n, l) / I(1,1, t-1)$ and let $J$ be the ideal $I(1,1, t) / I(1,1, t-1)$ in $R$.

We first prove that $R$ is a free $k[\mathbf{y}]$-module. The ideal $\left(a_{t}\right) \subseteq R$ is isomorphic to $R /\left(0:\left(a_{t}\right)\right)$. Since $I(1,1, t-1):\left(a_{t}\right)=I(1,1, t)$ in $R(n, l)$, we have $R /\left(0:\left(a_{t}\right)\right) \cong$ $R(n, l) / I(1,1, t)$, and the latter is a free $k[\mathbf{y}]$-module by hypothesis. The locus $V\left(a_{t}\right) \cap Y(1,1, t-1)$ is isomorphic to the preimage $\pi^{-1} Y_{n, L}(1,1, t-1)$ in the special arrangement $Z^{\prime}(n, 1)$ over $Z(n, L)$, where $L=[l] \backslash\{1\}$. The isomorphism is given by transposing the indices 1 and $t$ in $[l]$. Lemma 4.9.7 then implies that $R / \sqrt{ }\left(a_{t}\right)$ is a free $k[\mathbf{y}]$-module. Let $B_{1}$ be a free $k[\mathbf{y}]$-module basis of $\left(a_{t}\right)$ and let $B_{2}$ be a basis of $R / \sqrt{ }\left(a_{t}\right)$. By Lemmas 4.4.2 and 4.6.8 we have $\sqrt{ }\left(a_{t}\right)_{Q}=\left(a_{t}\right)_{Q}$ for $Q \in \hat{U}_{2}$, so $B_{1} \cup B_{2}$ is a basis of $R_{Q}$. Then $R$ is a free $k[\mathbf{y}]$-module by Lemma 4.4.9.

Now take $Z^{\prime}, Z^{\prime \prime}$ and $Z_{0}$ as in the preamble to Lemma 4.10.6 so $Z^{\prime}$ and $Z^{\prime \prime}$ are special arrangements over $Z(n, L)$, where $L=[l] \backslash\{t\}$. Set $R_{0}=\mathcal{O}\left(Z_{0}\right)$ and $R^{\prime}=\mathcal{O}\left(Z^{\prime}\right)$, and let $J^{\prime \prime}=\theta I\left(Z^{\prime \prime}\right) / I\left(Z^{\prime}\right)$ be the ideal of $\theta Z^{\prime \prime}$ as a closed subscheme of $Z^{\prime}=\theta Z^{\prime}$.

By (151) we have $I\left(Z_{0}\right) \cap J=0$ in $R$ so the canonical map $J \rightarrow J R_{0}$ is an isomorphism. Similarly, applying $\theta$ to (149), we have $I\left(Z_{0}\right) \cap \theta I\left(Z^{\prime \prime}\right)=0$ in $R^{\prime}$, hence $J^{\prime \prime} \rightarrow J^{\prime \prime} R_{0}$ is an isomorphism as well.

Since $R$ and $R / J$ are both free $k[\mathbf{y}]$-modules, finitely generated and $y$-graded in each $x$-degree, it follows that $J$ is a free $k[\mathbf{y}]$-module. Hence $J R_{0}$ is a free $k[\mathbf{y}]$ module. By Lemma 4.9.8, $I\left(Z^{\prime \prime}\right) / I\left(Z^{\prime}\right)$ is a free $k[\mathbf{y}]$-module with a common basis $B$ for the ideals $I \cap I\left(Z^{\prime \prime}\right) / I\left(Z^{\prime}\right)$, where $I=I_{n, L}(m, r, k) R^{\prime}$. In particular, this implies that $J^{\prime \prime}$ and $J^{\prime \prime} R_{0}$ are free $k[\mathbf{y}]$-modules. By Lemma 4.5.2, $R_{0}$ is a torsionfree $k[\mathbf{y}]$-module. By Lemmas 4.4 .2 and 4.10.6 the free submodules $J R_{0}$ and $J^{\prime \prime} R_{0}$ of $R_{0}$ coincide when localized at $Q \in \hat{U}_{2}$, so by Corollary 4.4.10 they are equal. Let $J_{0}=J R_{0}=J^{\prime \prime} R_{0}$.

With $B$ as in the previous paragraph, $\theta B$ is a free $k[\mathbf{y}]$-module basis of the ideal $J^{\prime \prime}$ in $R^{\prime}$, with subsets spanning the ideals $J^{\prime \prime} \cap \theta I_{n, L}(m, r, k) R^{\prime}$. We have canonical isomorphisms $J^{\prime \prime} \cong J_{0} \cong J$. Let $B^{\prime \prime} \subseteq J$ be the image of $\theta B$ under the composite isomorphism $J^{\prime \prime} \cong J$.

The canonical isomorphism $J^{\prime \prime} \rightarrow J_{0}$ is given by restriction to $Z_{0}$ of functions on $Z^{\prime}$ vanishing on $\theta Z^{\prime \prime}$. Lemma 4.9.7 implies that each ideal $\theta I_{n, L}(m, r, k) R^{\prime}$ is equal to its radical, namely, the ideal $I\left(\pi^{-1} \theta Y_{n, L}(m, r, k)\right)$ of the reduced preimage of $\theta Y_{n, L}(m, r, k)$ in $Z^{\prime}$. Therefore, since every function $p \in J^{\prime \prime}$ vanishes on $\theta Z^{\prime \prime}, p$ belongs to $\theta I_{n, L}(m, r, k) R^{\prime}$ if and only if its restriction to $Z_{0}$ vanishes on $Z_{0} \cap \pi^{-1} \theta Y_{n, L}(m, r, k)$. Similarly, writing $\pi_{1}$ for the coordinate projection $Y(1,1, t-1) \subseteq Z(n, l) \rightarrow Z(n, L)$, a function $p \in J$ belongs to the ideal $J \cap I\left(\pi_{1}^{-1} \theta Y_{n, L}(m, r, k)\right)$ in $R$ if and only if the same criterion holds (note that $\pi$ and $\pi_{1}$ have the same restriction to $Z_{0}$ ). This shows that the isomorphism $J^{\prime \prime} \cong J$ carries $J^{\prime \prime} \cap \theta I_{n, L}(m, r, k) R^{\prime}$ onto $J \cap I\left(\pi_{1}^{-1} \theta Y_{n, L}(m, r, k)\right)$. Hence the latter ideals are spanned by subsets of $B^{\prime \prime}$. 
Given $r>0, m$ and $k$, set $m^{\prime}=m-1, k^{\prime}=k$ if $k<t$, or else $m^{\prime}=$ $m, k^{\prime}=k-1$ if $k \geq t$. By Lemma 4.7.3. we have $Y(1,1, t) \cup(Y(m, r, k) \cap$ $Y(1,1, t-1))=Y(1,1, t) \cup \pi_{1}^{-1} \theta Y_{n, L}\left(m^{\prime}, r-1, k^{\prime}\right)$. The ideal in $R$ of $Y(1,1, t) \cup$ $\pi_{1}^{-1} \theta Y_{n, L}\left(m^{\prime}, r-1, k^{\prime}\right)$ is $J \cap I\left(\pi_{1}^{-1} \theta Y_{n, L}\left(m^{\prime}, r-1, k^{\prime}\right)\right)$, and we have shown above that it is spanned by a subset of $B^{\prime \prime}$. But this ideal is equal to the ideal of $Y(1,1, t) \cup(Y(m, r, k) \cap Y(1,1, t-1))$, namely, $J \cap \sqrt{ } I(m, r, k) R$. This shows that all the ideals $J \cap \sqrt{ } I(m, r, k) R$ are spanned by subsets of $B^{\prime \prime}$, the cases with $r=0$ being trivial. Since $R / J$ has a common ideal basis by hypothesis, the conclusion now follows from Corollary 4.10 .4 .

4.11. Proof and consequences of Theorem 4.2. We have established all the stages in our basis construction procedure in 4.10 To complete the proof of Theorem 4.2 and its corollary Theorem 4.1, we have only to assemble the pieces.

Proof of Theorem 4.2. We prove the theorem by induction on $n$ and $l$, maintaining as part of the induction hypothesis that the common ideal basis specified in the theorem is homogeneous. The base case $l=0$ is given by Lemma 4.3.2, The base case for $n$ is $n=1$. Note that for $n=1$ we have $Z(1, l) \cong Z(1,0)=E$, for all $l$, and that the only nontrivial $Y(m, r, k)$ is $Y(1,1,0)$, which already appears in $Z(1,0)$. Thus the case $n=1$ is included in the case $l=0$.

For $n>1$ and $l>0$ we can assume by induction that $R(n-1, l)$ has a homogeneous common ideal basis. Then by Lemma 4.10.2, so does $R(n, l) / I(1,1, l)$. We can also assume by induction that $R(n, l-1)$ has a homogeneous common ideal basis. Applying Lemma 4.10.7 repeatedly, with $t$ descending from $l$ to 1 , we conclude that $R(n, l) / I(1,1,0)$ has a homogeneous common ideal basis. Then by Lemma 4.10.5, so does $R(n, l)$.

The bridge from Theorem 4.1 to Hilbert schemes is supplied by Proposition 3.8.1 which we restate and prove below. We remark that the bridge is not quite as narrow as it might appear. The polygraph rings $R(n, l)$ carry geometric information about the tensor powers $\rho^{*} B^{\otimes l}$ of the tautological bundle over the isospectral Hilbert scheme $X_{n}$. Theorem 4.1 can be further exploited to obtain vanishing theorems for these vector bundles. This subject will be taken up elsewhere, along with its application to the determination of the character formula for diagonal harmonics, which was discussed briefly in the introduction.

Proposition 4.11.1. Let $J=\mathbb{C}[\mathbf{x}, \mathbf{y}] A$ be the ideal generated by the space of alternating polynomials $A=\mathbb{C}[\mathbf{x}, \mathbf{y}]^{\epsilon}$. Then $J^{d}$ is a free $\mathbb{C}[\mathbf{y}]$-module for all $d$.

Proof. Set $l=n d$, and let $Z(n, l)$ be the polygraph over $\mathbb{C}$, a subspace arrangement in $\left(\mathbb{C}^{2}\right)^{n} \times\left(\mathbb{C}^{2}\right)^{l}$. Let $G=S_{n}^{d}$ be the Cartesian product of $d$ copies of the symmetric group $S_{n}$, acting on $\left(\mathbb{C}^{2}\right)^{n} \times\left(\mathbb{C}^{2}\right)^{l}$ by permuting the factors in $\left(\mathbb{C}^{2}\right)^{l}$ in $d$ consecutive blocks of length $n$. In other words, each $w \in G$ fixes the coordinates $\mathbf{x}, \mathbf{y}$ on $\left(\mathbb{C}^{2}\right)^{n}$, and for each $k=0, \ldots, d-1$ permutes the coordinate pairs $a_{k n+1}, b_{k n+1}$ through $a_{k n+n}, b_{k n+n}$ among themselves.

Let $R(n, l)=\mathbb{C}[\mathbf{x}, \mathbf{y}, \mathbf{a}, \mathbf{b}] / I(n, l)$ be the coordinate ring of $Z(n, l)$. By Theorem 4.1. $R(n, l)$ is a free $\mathbb{C}[\mathbf{y}]$-module. By the symmetry of its definition, $I(n, l)$ is a $G$-invariant ideal, so $G$ acts on $R(n, l)$. We claim that $J^{d}$ is isomorphic as a $\mathbb{C}[\mathbf{x}, \mathbf{y}]$-module to the space $R(n, l)^{\epsilon}$ of $G$-alternating elements of $R(n, l)$. Each $x$-degree homogeneous component of $R(n, l)$ is a finitely generated $y$-graded free 
$\mathbb{C}[\mathbf{y}]$-module. Since $R(n, l)^{\epsilon}$ is a graded direct summand of $R(n, l)$, it is a free $\mathbb{C}[\mathbf{y}]$-module, so the claim proves the proposition.

Let $f_{0}:[l] \rightarrow[n]$ be defined by $f_{0}(k n+i)=i$ for all $0 \leq k<d, 1 \leq i \leq n$. Restriction of regular functions from $Z(n, l)$ to its component subspace $W_{f_{0}}$ is given by the $\mathbb{C}[\mathbf{x}, \mathbf{y}]$-algebra homomorphism $\psi: R(n, l) \rightarrow \mathbb{C}[\mathbf{x}, \mathbf{y}]$ mapping $a_{k n+i}, b_{k n+i}$ to $x_{i}, y_{i}$. Observe that $\psi$ maps $R(n, l)^{\epsilon}$ surjectively onto $\mathbb{C}[\mathbf{x}, \mathbf{y}] A^{d}=J^{d}$.

Let $p$ be an arbitrary element of $R(n, l)^{\epsilon}$. Since $p$ is $G$-alternating, $p$ vanishes on $W_{f}$ if $f(k n+i)=f(k n+j)$ for some $0 \leq k<d$ and some $1 \leq i<j \leq n$. Thus the regular function defined by $p$ on $Z(n, l)$ is determined by its restriction to those components $W_{f}$ such that for each $k$, the sequence $f(k n+1), \ldots, f(k n+n)$ is a permutation of $\{1, \ldots, n\}$. Moreover, for every such $f$ there is an element $w \in G$ carrying $W_{f}$ onto $W_{f_{0}}$. Hence $p$ is determined by its restriction to $W_{f_{0}}$. This shows that $p$ vanishes on $Z(n, l)$ if $\psi(p)=0$, that is, the kernel of the map $\psi: R(n, l)^{\epsilon} \rightarrow J^{d}$ is zero.

4.12. Arbitrary ground rings. For convenience of exposition we have assumed that $k$ is a field of characteristic zero. For completeness we now show that the results on polygraphs hold over any ground ring. This generalization is not needed elsewhere in the paper, where we always have $k=\mathbb{C}$.

To begin with, observe that the proof of Theorems 4.1 and 4.2 goes through with minor modifications for $k=\mathbb{Z}$. As a matter of notation, we must replace $k(\mathbf{y})$ throughout with $\mathbb{Q}(\mathbf{y})$. In Lemma 4.5.1 and elsewhere, we must read "generates as a $\mathbb{Z}$-module" for "spans as a $k$-vector space".

In the proofs of Lemmas 4.6.4, 4.6.8 and 4.9.5 we implicitly used the facts that every scheme over a field $k$ is flat over $k$, and that the product of reduced schemes over a field of characteristic zero is reduced. In our case, the schemes in question are (reduced) subspace arrangements in $E^{n} \times E^{l}$. If $Y=\bigcup Y_{\alpha}$ is a subspace arrangement defined over $\mathbb{Z}$, then its coordinate ring $R=\mathcal{O}(Y)$ is a subring of a direct sum $\bigoplus \mathcal{O}\left(Y_{\alpha}\right)$ of polynomial rings over $\mathbb{Z}$. Therefore $R$ is a torsion-free Abelian group, that is, a flat $\mathbb{Z}$-module. Every subspace arrangement over $\mathbb{Z}$ is thus flat, and a product of reduced schemes flat over $\mathbb{Z}$ is reduced.

In the proofs of Lemmas 4.4 .9 and 4.10 .3 we also used the fact that a rational function regular outside a subset of codimension two in Spec $k[\mathbf{y}]$ is regular. This holds with any normal integral domain in place of $k[\mathbf{y}]$, and in particular for $\mathbb{Z}[\mathbf{y}]$.

The theorem for $k=\mathbb{Z}$ implies the following fully general result.

Theorem 4.3. Let $E=\mathbb{A}^{2}(k)$, where $k$ is any commutative ring with unit. Let $Z(n, l) \subseteq E^{n} \times E^{l}$ be a polygraph over $k$ (defined as the union of the closed subschemes $W_{f}$, just as in 4.1.1]. Then the coordinate ring $R(n, l)=\mathcal{O}(Z(n, l))$ is a free $k[\mathbf{y}]$-module.

Proof. By the preceding remarks, the theorem holds for $k=\mathbb{Z}$. Tensoring the exact sequence

$$
0 \rightarrow I_{\mathbb{Z}}(n, l) \rightarrow \mathbb{Z}[\mathbf{x}, \mathbf{y}, \mathbf{a}, \mathbf{b}] \rightarrow R_{\mathbb{Z}}(n, l) \rightarrow 0,
$$

over $\mathbb{Z}[\mathbf{y}]$ with $k[\mathbf{y}]$, we get an exact sequence

$$
0 \rightarrow I=k[\mathbf{y}] I_{\mathbb{Z}}(n, l) \rightarrow k[\mathbf{x}, \mathbf{y}, \mathbf{a}, \mathbf{b}] \rightarrow R \rightarrow 0
$$

with $R$ a free $k[\mathbf{y}]$-module. Since $I_{\mathbb{Z}}(n, l) \subseteq I_{\mathbb{Z}}\left(W_{f}\right)$ for all $f$, and $I_{f}=k[\mathbf{y}] I_{\mathbb{Z}}\left(W_{f}\right)$ by (67), we have $I \subseteq I(n, l)$. 
Let $\delta=\prod_{i<j}\left(y_{i}-y_{j}\right)$. Note that $U_{1}$ is the affine open set $U_{\delta}$, so inverting $\delta$ is the same thing as localizing to $U_{1}$. By Lemma 4.4.3 therefore, we have $R_{\mathbb{Z}}(n, l)_{\delta}=$ $\bigoplus_{f} \mathcal{O}_{\mathbb{Z}}\left(W_{f}\right)_{\delta}$, and hence $R_{\delta}=\bigoplus_{f} \mathcal{O}\left(W_{f}\right)_{\delta}$. By definition $I(n, l)$ is the kernel of the canonical homomorphism

$$
k[\mathbf{x}, \mathbf{y}, \mathbf{a}, \mathbf{b}] \rightarrow \bigoplus_{f} \mathcal{O}\left(W_{f}\right) .
$$

Hence inverting $\delta$ in (162) gives $I_{\delta}=I(n, l)_{\delta}$.

Now $\delta$ is a non-zero-divisor in $k[\mathbf{y}]$, so it is also a non-zero-divisor on the free $k[\mathbf{y}]$ module $R$ and its submodule $I(n, l) / I$. But since $(I(n, l) / I)_{\delta}=0$, every element of $I(n, l) / I$ is annihilated by a power of $\delta$. Therefore we have $I(n, l) / I=0$ and $R=R(n, l)$.

\section{Applications, conjectures and problems}

5.1. The $G$-Hilbert scheme. Let $G \subseteq \mathrm{GL}(V)$ be a finite group acting faithfully on a complex vector space $V=\mathbb{C}^{m}$. For all vectors $v$ in a nonempty open subset of $V$, the orbit $G v$ consists of $N=|G|$ distinct points and thus represents an element of $\operatorname{Hilb}^{N}(V)$. The closure in $\operatorname{Hilb}^{N}(V)$ of the set of such orbits is a component of the $G$-fixed locus of $\operatorname{Hilb}^{N}(V)$, called the Hilbert scheme of $G$-orbits or $G$-Hilbert scheme and denoted $V / / G$. These schemes were first defined and studied by Ito and Nakamura [28, 29].

The universal family over $V / / G$ has a $G$-action, in which the coordinate ring of each fiber affords the regular representation of $G$. There is a canonical Chow morphism

$$
V / / G \rightarrow V / G
$$

making the $G$-Hilbert scheme $V / / G$ projective and birational over $V / G$. In the case $V=\left(\mathbb{C}^{2}\right)^{n}, G=S_{n}$, as shown in [24], $\S 4$, the morphism in (164) factors through a morphism

$$
\phi:\left(\mathbb{C}^{2}\right)^{n} / / S_{n} \rightarrow H_{n}
$$

of schemes over $S^{n} \mathbb{C}^{2}$. In fact, if $Y$ denotes the universal family over $\left(\mathbb{C}^{2}\right)^{n} / / S_{n}$, then $Y / S_{n-1}$ is a flat family of degree $n$, and it can be naturally identified with a family of subschemes of $\mathbb{C}^{2}$. The universal property of $H_{n}$ then gives rise to $\phi$.

By Theorem 3.1, the isospectral Hilbert scheme is a flat family $X_{n} \subseteq H_{n} \times$ $\left(\mathbb{C}^{2}\right)^{n}$ of degree $n$ !. Hence the universal property gives rise to a morphism $H_{n} \rightarrow$ Hilb ${ }^{n !}\left(\left(\mathbb{C}^{2}\right)^{n}\right)$ whose image is clearly contained in $\left(\mathbb{C}^{2}\right)^{n} / / S_{n}$. This morphism is inverse to $\phi$, since it is so on the generic locus. By construction, $X_{n}$ is pulled back via this $\phi^{-1}$ from the universal family $Y$ over $\left(\mathbb{C}^{2}\right)^{n} / / S_{n}$. Hence we have the following result, which is in fact equivalent to the flatness of $\rho$ and thus to the Cohen-Macaulay property of $X_{n}$.

Theorem 5.1. The morphism $\phi$ in (165) is an isomorphism of $\left(\mathbb{C}^{2}\right)^{n} / / S_{n}$ onto $H_{n}$, and it identifies the universal family over $\left(\mathbb{C}^{2}\right)^{n} / / S_{n}$ with the isospectral Hilbert scheme $X_{n}$.

In light of Theorem 5.1 the vector bundle $P=\rho_{*} \mathcal{O}_{X_{n}}$ considered in the proof of Proposition 3.7.4 may be identified with the tautological bundle on $\left(\mathbb{C}^{2}\right)^{n} / / S_{n}$, a vector bundle whose fibers afford the regular representation of $S_{n}$. To each irreducible representation $V^{\lambda}$ of $S_{n}$ is associated a vector bundle $C_{\lambda}=\operatorname{Hom}_{S_{n}}\left(V^{\lambda}, P\right)$ 
on $\left(\mathbb{C}^{2}\right)^{n} / / S_{n}$ known as a character sheaf. We have $P=\bigoplus_{\lambda} C_{\lambda} \otimes V^{\lambda}$, and since $P$ affords the regular representation, $C_{\lambda}$ has rank $\operatorname{dim}\left(V^{\lambda}\right)=\chi^{\lambda}(1)$. It follows from Theorem 3.1. Proposition 3.7.3 and Theorem 3.2 that the Kostka-Macdonald coefficient $\tilde{K}_{\lambda \mu}(q, t)$ may be interpreted as the doubly graded Hilbert series of the fiber of $C_{\lambda}$ at the $\mathbb{T}^{2}$-fixed point $I_{\mu}$. Equivalently, $\tilde{K}_{\lambda \mu}(q, t)$ is the character of the $\mathbb{T}^{2}$ action on this fiber.

We remark that the geometric picture described by Theorems 3.1 and 5.1 applies more generally with any smooth complex quasiprojective surface $E$ replacing $\mathbb{C}^{2}$. Specifically, we have that $E^{n} / / S_{n}$ is isomorphic to the Hilbert scheme $\operatorname{Hilb}^{n}(E)$, and the universal family over $E^{n} / / S_{n}$ may be identified with the isospectral Hilbert scheme $X_{n}(E)$, which is also the blowup of $E^{n}$ with center the union of the pairwise diagonals. To see this, note that Lemma 3.3.1 holds in this situation and reduces the question to a local one over points of the form $(P, P, \ldots, P) \in E^{n}$, for $P \in E$. Passing to the completion with respect to the ideal of such a point, we see that the Cohen-Macaulay property of $X_{n}(E)$ and its identification with the blowup are equivalent to the corresponding facts for $X_{n}\left(\mathbb{C}^{2}\right)$.

Returning to the general situation, assume that $G$ is a subgroup of $\mathrm{SL}(V)$. Then $V / G$ is Gorenstein and has rational singularities. A desingularization

$$
\sigma: H \rightarrow V / G
$$

is said to be crepant if $\omega_{H}=\mathcal{O}_{H}$. The generalized McKay correspondence, conjectured by Reid [43] and proved by Batyrev [1], asserts that if $H$ is a crepant resolution, then the sum of the Betti numbers of $H$ is equal to the number of conjugacy classes, or the number of irreducible characters, of $G$. For $V=\left(\mathbb{C}^{2}\right)^{n}$, $G=S_{n}$, the Hilbert scheme $H_{n}$ is a crepant resolution of $S^{n} \mathbb{C}^{2}$, by Proposition 3.6.3. The computation of the homology of $H_{n}$ by Ellingsrud and Strømme [11] verifies the McKay correspondence in this case.

In dimension 2, it develops that $V / / G$ is a crepant resolution of $V / G$ for every $G$. Nakamura conjectured that this should hold in dimension 3 as well, and proved it for $G$ abelian [42]. Recently, Bridgeland, King and Reid [6] established Nakamura's conjecture by proving that a certain fiber dimension condition on the map $V / / G \rightarrow V / G$ is sufficient to imply that $V / / G$ is a crepant resolution of $V / G$. In this situation, moreover, the McKay correspondence holds in a strong form, expressed as an equivalence of derived categories. By Theorem 5.1 we know that $V / / G$ is a crepant resolution of $V / G$ in the case $G=S_{n}, V=\left(\mathbb{C}^{2}\right)^{n}$. As things stand, this is the only known higher-dimensional family of groups for which $V / / G$ is a crepant resolution, apart from examples built up as products of lower-dimensional cases. It is natural to ask whether Theorem 5.1] might generalize to yield other such families.

Problem 5.1.1. Let $G$ be a complex reflection group with defining representation $W$, and let $V=W \oplus W^{*}$. For which $G$ is $V / / G$ a crepant resolution of $V / G$ ?

The related problem of whether $\left(W \oplus W^{*}\right) / G$ admits a crepant resolution at all has been considered by Kaledin [30] and Verbitsky [47], who prove that this can happen only for complex reflection groups.

We have limited information about the solution to Problem[5.1.1 By our results here, the symmetric groups, or Weyl groups of type $A$, are examples for which $V / / G$ is a crepant resolution. By the classical McKay correspondence for subgroups of $\mathrm{SL}(2)$, the cyclic groups $\mathbb{Z}_{n}$, regarded as one-dimensional complex reflection groups, are also examples. The hyperoctahedral groups, or Weyl groups of type $B$, 
are counterexamples. This can be seen by explicit computation for $B_{2}$. Since $B_{2}$ occurs as the stabilizer of a vector in the reflection representation of $B_{n}$, it follows that $B_{n}$ is a counterexample for all $n \geq 2$.

For the hyperoctahedral groups $G=B_{n}$, and more generally for $G$ the wreath product of the symmetric group $S_{n}$ with the cyclic group $\mathbb{Z}_{m}$, crepant resolutions of $\left(W \oplus W^{*}\right) / G$ do exist. In these cases, the action of $G$ on $V=W \oplus W^{*}$ is the wreath product action on $\left(\mathbb{C}^{2}\right)^{n}$, where the cyclic group $\mathbb{Z}_{m}$ acts on $\mathbb{C}^{2}=\mathbb{C} \oplus \mathbb{C}^{*}$ as the direct sum of a one-dimensional representation and its dual. As observed by Wang [4], whenever $G$ acts on $V=\left(\mathbb{C}^{2}\right)^{n}$ as the wreath product of $S_{n}$ with a finite subgroup $\Gamma \subseteq \mathrm{SL}(2)$, there are two natural crepant resolutions. One is a component of the $\Gamma$-fixed locus in the Hilbert scheme $H_{n|\Gamma|}$. The other is the Hilbert scheme $\operatorname{Hilb}^{n}\left(X_{\Gamma}\right)$ of the minimal resolution $X_{\Gamma}=\mathbb{C}^{2} / / \Gamma$ of $\mathbb{C}^{2} / \Gamma$. In general (and for $G=B_{2}$ in particular) these two resolutions can be different, and neither of them need coincide with $V / / G$.

5.2. Higher dimensions. For $d>2$ the Hilbert scheme $\operatorname{Hilb}^{n}\left(\mathbb{C}^{d}\right)$ has in general multiple irreducible components, frequently of dimension exceeding $d n$, and bad singularities. Nevertheless there is a distinguished principal component $H_{n}\left(\mathbb{C}^{d}\right)$, the closure of the open subset parametrizing reduced subschemes $S \subseteq \mathbb{C}^{d}$ with $n$ distinct points. The isospectral Hilbert scheme $X_{n}\left(\mathbb{C}^{d}\right)$ over $H_{n}\left(\mathbb{C}^{d}\right)$ can be defined as in 3.2.4 and the analogs of Propositions 3.4.1 and 3.4.2 hold also for $d>2$.

Conjecture 5.2.1. For all $d$ and $n$, the isospectral Hilbert scheme $X_{n}\left(\mathbb{C}^{d}\right)$ over the principal component $H_{n}\left(\mathbb{C}^{d}\right)$ of $\mathrm{Hilb}^{n}\left(\mathbb{C}^{d}\right)$ is normal and Cohen-Macaulay.

Since $H_{n}\left(\mathbb{C}^{d}\right)=X_{n}\left(\mathbb{C}^{d}\right) / S_{n}$, the conjecture implies that the principal component $H_{n}\left(\mathbb{C}^{d}\right)$ is itself normal and Cohen-Macaulay. Note that it does not imply the analog of the $n$ ! conjecture in $d$ sets of variables, since in general $H_{n}\left(\mathbb{C}^{d}\right)$ is singular and thus the projection $\rho: X_{n}\left(\mathbb{C}^{d}\right) \rightarrow H_{n}\left(\mathbb{C}^{d}\right)$ need not be flat. In other words, Theorem 5.1 can fail, and $H_{n}\left(\mathbb{C}^{d}\right)$ need no longer coincide with the $G$-Hilbert scheme $\left(\mathbb{C}^{d}\right)^{n} / / S_{n}$ for $d>2$.

An example exhibiting the failure of the higher-dimensional analogs of the $n$ ! conjecture and Theorem 5.1 occurs in dimension $d=3$, with $n=4$. The analog of the $n$ ! conjecture would require the determinant

$$
\Delta(\mathbf{x}, \mathbf{y}, \mathbf{z})=\operatorname{det}\left[\begin{array}{llll}
1 & x_{1} & y_{1} & z_{1} \\
1 & x_{2} & y_{2} & z_{2} \\
1 & x_{3} & y_{3} & z_{3} \\
1 & x_{4} & y_{4} & z_{4}
\end{array}\right]
$$

to have $4 !=24$ linearly independent partial derivatives, but in fact it has only 20 . More generally, Tesler [46] has shown that the analogous determinant in dimension $d$ has $\left(\begin{array}{c}2 d \\ d\end{array}\right)$ independent derivatives.

Nevertheless, Conjecture 5.2.1 does hold for the case $d=3, n=4$, as we have verified using the computer algebra system Macaulay $\left[2\right.$. Both $X_{4}\left(\mathbb{C}^{3}\right)$ and $H_{4}\left(\mathbb{C}^{3}\right)$ turn out to be normal and Gorenstein. (Strictly speaking, we have verified this over a field of characteristic 31991, Macaulay's default, and of course modulo the correctness of the program.)

Conjecture 5.2.1 is rather speculative, and the author would not be greatly surprised if it turned out to be wrong. A more definite conjecture, whose failure he would indeed consider a surprise, is the following higher-dimensional version of the polygraph theorem. 
Conjecture 5.2.2. The coordinate ring of the polygraph $Z(n, l)$ over $E=\mathbb{A}^{d}$ is a free $k[\mathbf{z}]$-module, where $\mathbf{x}, \mathbf{y}, \ldots, \mathbf{z}=x_{1}, y_{1}, \ldots, z_{1}, \ldots, x_{n}, y_{n}, \ldots, z_{n}$ are the coordinates on $E^{n}$.

This conjecture implies the analogs of Proposition 3.8.1 and Corollary 3.8 .3 in more than two sets of variables, with essentially the same proof. Hence it implies that $X_{n}\left(\mathbb{C}^{d}\right)$ is the blowup of $\left(\mathbb{C}^{d}\right)^{n}$ with center the reduced union of the pairwise diagonals, that it is arithmetically normal in its embedding as a blowup, and that it is flat over the coordinate space $\mathbb{C}^{n}=\operatorname{Spec} \mathbb{C}[\mathbf{z}]$ in any one set of the variables.

5.3. Bases. The proof of the $n$ ! conjecture via Proposition 3.7 .3 and Theorem 3.1 does not yield an explicit basis of the space $D_{\mu}$ or the ring $R_{\mu}$. We still have an important combinatorial open problem.

Problem 5.3.1. Find a doubly homogeneous basis of $R_{\mu}$, compatible with (or triangular with respect to) the decomposition into isotypic components as an $S_{n^{-}}$ module, and indexed combinatorially so as to yield a purely combinatorial interpretation of the Kostka-Macdonald coefficients $\tilde{K}_{\lambda \mu}(q, t)$.

A related doubly graded $S_{n}$-module is the ring $R_{n}=\mathbb{C}[\mathbf{x}, \mathbf{y}] / I_{n}$, where $I_{n}$ is the ideal generated by all $S_{n}$-invariant polynomials without constant term. This ring has the same graded character as the space of diagonal harmonics, and is the subject of a series of conjectures relating its character to various combinatorial objects and to Macdonald polynomials [16, 22]. In particular it is conjectured that $\operatorname{dim}_{\mathbb{C}} R_{n}=(n+1)^{n-1}$.

Each ring $R_{\mu}$ for $\mu$ a partition of $n$ is a quotient ring of $R_{n}$, so bases of $R_{\mu}$ may be realized as subsets of a basis of $R_{n}$.

Problem 5.3.2. Find a doubly homogeneous basis of $R_{n}$ compatible with the $S_{n^{-}}$ module structure, and for each partition $\mu$ of $n$, a distinguished subset which is a basis of $R_{\mu}$.

Algebraically, the relationship between $R_{n}$ and $R(n, l)$ can be described as follows. Let $l=n$. In the polygraph $Z(n, n)$ we have a subarrangement

$$
Z=\bigcup W_{f}, \quad \text { over permutations } f:[n] \rightarrow[n] .
$$

Let $J \subseteq R(n, n)$ be the ideal of $Z$. For any $S_{n}$-invariant polynomial $p(\mathbf{x}, \mathbf{y})$ we clearly have $p(\mathbf{a}, \mathbf{b})-p(\mathbf{x}, \mathbf{y}) \in J$. Hence if $p \in I_{n}$ is an $S_{n}$-invariant polynomial without constant term, then we have $p(\mathbf{a}, \mathbf{b}) \in J+(\mathbf{x}, \mathbf{y})$. This yields a ring homomorphism $\psi: R_{n} \rightarrow R(n, n) /(J+(\mathbf{x}, \mathbf{y}))$, sending $\mathbf{x}, \mathbf{y}$ in $R_{n}$ to $\mathbf{a}, \mathbf{b}$ in $R(n, n) /(J+(\mathbf{x}, \mathbf{y}))$. We conjecture that $\psi$ is an isomorphism. Postulating this for the moment, we have a surjection of $R(n, n) /(\mathbf{x}, \mathbf{y})$ onto a copy of $R_{n}$ in the variables $\mathbf{a}, \mathbf{b}$.

From the proof of Theorem 4.2, we have an at least somewhat explicit free $k[\mathbf{y}]$ module basis of $R(n, n)$, and therefore a vector space basis of $R(n, n) /(\mathbf{y})$. This basis is not compatible with the $S_{n}$-action, nor does a subset of it span the ideal $(\mathbf{x})$. Nevertheless, some subset of it must be a basis of $R(n, n) /(\mathbf{x}, \mathbf{y})$, and some further subset must be a basis of $R(n, n) /(J+(\mathbf{x}, \mathbf{y}))$.

Problem 5.3.3. (1) Find a distinguished subset of the free $k[\mathbf{y}]$-module basis of $R(n, l)$ constructed in the proof of Theorem 4.2 which is a vector space basis of $R(n, l) /(\mathbf{x}, \mathbf{y})$. 
(2) For $l=n$, find a further distinguished subset of the above basis, with $(n+1)^{n-1}$ elements, such that setting $\mathbf{x}=\mathbf{y}=0$ and then replacing $a_{1}, b_{1}, \ldots, a_{n}, b_{n}$ with $x_{1}, y_{1}, \ldots, x_{n}, y_{n}$, we get a basis of $R_{n}$. To agree with the conjectures on $R_{n}$ discussed in [22], this basis should be indexed by parking functions in such a way that the $x$-degree of the basis element is the weight of the parking function.

(3) For each $\mu$, find a still further distinguished subset of the above basis of $R_{n}$, with $n$ ! elements, whose image in $R_{\mu}$ is a basis. For each $\lambda$, distinguish yet another subset whose projection on the isotypic component of $R_{\mu}$ affording the $S_{n}$ character $\chi^{\lambda}$ is a basis of that isotypic component, so that the enumerator of its elements by $x$ - and $y$-degrees is equal to $\chi^{\lambda}(1) \tilde{K}_{\lambda \mu}(q, t)$.

\section{REFERENCES}

[1] Victor V. Batyrev, Non-Archimedean integrals and stringy Euler numbers of log-terminal pairs, J. Eur. Math. Soc. (JEMS) 1 (1999), no. 1, 5-33, arXiv:math.AG/9803071. CMP 99:09

[2] David Bayer and Michael Stillman, Macaulay: A computer algebra system for algebraic geometry, Version 3.0, Software distributed via ftp: math.columbia.edu/pub/bayer/Macaulay, 1994.

[3] F. Bergeron, A. M. Garsia, M. Haiman, and G. Tesler, Identities and positivity conjectures for some remarkable operators in the theory of symmetric functions, Methods Appl. Anal. 6 (1999), no. 3, 363-420. CMP 2001:07

[4] N. Bergeron and A. M. Garsia, On certain spaces of harmonic polynomials, Hypergeometric functions on domains of positivity, Jack polynomials, and applications (Tampa, FL, 1991), Amer. Math. Soc., Providence, RI, 1992, pp. 51-86. MR 94a:05212

[5] Joël Briançon, Description de $\operatorname{Hilb}^{n} C\{x, y\}$, Invent. Math. 41 (1977), no. 1, 45-89. MR 56:15637

[6] Tom Bridgeland, Alastair King, and Miles Reid, The McKay correspondence as an equivalence of derived categories, J. Amer. Math. Soc. 14 (2001), no. 3, 555-578.

[7] William Brockman and Mark Haiman, Nilpotent orbit varieties and the atomic decomposition of the q-Kostka polynomials, Canad. J. Math. 50 (1998), no. 3, 525-537. MR 2000c:05153

[8] Jan Cheah, Cellular decompositions for nested Hilbert schemes of points, Pacific J. Math. 183 (1998), no. 1, 39-90. MR 99d:14002

[9] Ivan Cherednik, Double affine Hecke algebras and Macdonald's conjectures, Ann. of Math. (2) 141 (1995), no. 1, 191-216. MR 96m:33010

[10] Corrado De Concini and Claudio Procesi, Symmetric functions, conjugacy classes and the flag variety, Invent. Math. 64 (1981), no. 2, 203-219. MR 82m:14030

[11] Geir Ellingsrud and Stein Arild Strømme, On the homology of the Hilbert scheme of points in the plane, Invent. Math. 87 (1987), no. 2, 343-352. MR 88c:14008

[12] Jacques Emsalem, Géométrie des points épais, Bull. Soc. Math. France 106 (1978), no. 4, 399-416. MR 80j:14008

[13] Pavel I. Etingof and Alexander A. Kirillov, Jr., Macdonald's polynomials and representations of quantum groups, Math. Res. Lett. 1 (1994), no. 3, 279-296, hep-th/9312103. MR 96m:17025

[14] John Fogarty, Algebraic families on an algebraic surface, Amer. J. Math 90 (1968), 511-521. MR 38:5778

[15] A. M. Garsia and M. Haiman, A graded representation model for Macdonald's polynomials, Proc. Nat. Acad. Sci. U.S.A. 90 (1993), no. 8, 3607-3610. MR 94b:05206

[16] — A remarkable $q, t$-Catalan sequence and $q$-Lagrange inversion, J. Algebraic Combin. 5 (1996), no. 3, 191-244. MR 97k:05208

[17] A. M. Garsia and C. Procesi, On certain graded $S_{n}$-modules and the $q$-Kostka polynomials, Adv. Math. 94 (1992), no. 1, 82-138. MR 93j:20030

[18] A. M. Garsia and J. Remmel, Plethystic formulas and positivity for q, t-Kostka coeffcients, Mathematical essays in honor of Gian-Carlo Rota (Cambridge, MA, 1996), Birkhäuser Boston, Boston, MA, 1998, pp. 245-262. MR 99j:05189d

[19] A. M. Garsia and G. Tesler, Plethystic formulas for Macdonald q, t-Kostka coefficients, Adv. Math. 123 (1996), no. 2, 144-222. MR 99j:05189e 
[20] Mark L. Green, Generic initial ideals, Six lectures on commutative algebra (Bellaterra, 1996), Birkhäuser, Basel, 1998, pp. 119-186. MR 99m:13040

[21] Alexander Grothendieck, Techniques de construction et théorèmes d'existence en géométrie algébrique. IV. Les schémas de Hilbert (Exp. no. 221), Séminaire Bourbaki, Vol. 6, Année 1960/61, Soc. Math. France, Paris, 1995, pp. 249-276. MR 99f:00038

[22] Mark Haiman, Conjectures on the quotient ring by diagonal invariants, J. Algebraic Combin. 3 (1994), no. 1, 17-76. MR 95a:20014

[23] _ t, q-Catalan numbers and the Hilbert scheme, Discrete Math. 193 (1998), no. 1-3, 201-224, Selected papers in honor of Adriano Garsia (Taormina, 1994). MR 2000k:05264

[24] _ Macdonald polynomials and geometry, New perspectives in geometric combinatorics (Billera, Björner, Greene, Simion, and Stanley, eds.), MSRI Publications, vol. 38, Cambridge University Press, 1999, pp. 207-254. CMP 2000:07

[25] Robin Hartshorne, Residues and duality, Springer-Verlag, Berlin, 1966, Lecture notes of a seminar on the work of A. Grothendieck, given at Harvard 1963/64. With an appendix by P. Deligne. Lecture Notes in Mathematics, Vol. 20. MR 36:5145

[26] Local cohomology, Springer-Verlag, Berlin, 1967, A seminar given by A. Grothendieck, Harvard University, Fall, 1961. Lecture Notes in Mathematics, Vol. 41. MR 37:219

[27] R. Hotta and T. A. Springer, A specialization theorem for certain Weyl group representations and an application to the Green polynomials of unitary groups, Invent. Math. 41 (1977), no. 2, 113-127. MR 58:5945

[28] Y. Ito and I. Nakamura, McKay correspondence and Hilbert schemes, Proc. Japan Acad. Ser. A Math. Sci. 72 (1996), no. 7, 135-138. MR 97k:14003

[29] _ Hilbert schemes and simple singularities, New trends in algebraic geometry (Warwick, 1996), Cambridge Univ. Press, Cambridge, 1999, pp. 151-233. MR 2000i:14004]

[30] D. Kaledin, McKay correspondence for symplectic quotient singularities, Electronic preprint, arXiv:math.AG/9907087, 1999.

[31] Shin-ichi Kato, Spherical functions and a q-analogue of Kostant's weight multiplicity formula, Invent. Math. 66 (1982), no. 3, 461-468. MR 84b:22030

[32] Anatol N. Kirillov and Masatoshi Noumi, Affine Hecke algebras and raising operators for Macdonald polynomials, Duke Math. J. 93 (1998), no. 1, 1-39, arXiv:q-alg/9605004. MR 99j:05189a

[33] Friedrich Knop, Integrality of two variable Kostka functions, J. Reine Angew. Math. 482 (1997), 177-189, arXiv:q-alg/9603027. MR 99j:05189c

[34] Hanspeter Kraft, Conjugacy classes and Weyl group representations, Young tableaux and Schur functions in algebra and geometry (Toruń, 1980), Soc. Math. France, Paris, 1981, pp. 191-205. MR 83h:20017

[35] Luc Lapointe and Luc Vinet, Operator construction of the Jack and Macdonald symmetric polynomials, Special functions and differential equations (Madras, 1997), Allied Publ., New Delhi, 1998, pp. 271-279. MR 2000a:05210

[36] Alain Lascoux and Marcel-Paul Schützenberger, Sur une conjecture de H. O. Foulkes, C. R. Acad. Sci. Paris Sér. A-B 286 (1978), no. 7, A323-A324. MR 57:12672

[37] G. Lusztig, Green polynomials and singularities of unipotent classes, Adv. in Math. 42 (1981), no. 2, 169-178. MR 83c:20059

[38] _ Singularities, character formulas, and a q-analog of weight multiplicities, Analysis and topology on singular spaces, II, III (Luminy, 1981), Soc. Math. France, Paris, 1983, pp. 208-229. MR 85m:17005

[39] I. G. Macdonald, A new class of symmetric functions, Actes du 20e Séminaire Lotharingien, vol. 372/S-20, Publications I.R.M.A., Strasbourg, 1988, pp. 131-171.

[40] _ Symmetric functions and Hall polynomials, second ed., The Clarendon Press, Oxford University Press, New York, 1995, With contributions by A. Zelevinsky, Oxford Science Publications. MR 96h:05207

[41] — Affine Hecke algebras and orthogonal polynomials, Astérisque 237 (1996), 189-207, Séminaire Bourbaki 1994/95, Exp. no. 797. MR 99f:33024

[42] I. Nakamura, Hilbert schemes of abelian group orbits, Journal of Algebraic Geometry, to appear.

[43] M. Reid, McKay correspondence, Electronic preprint, arXiv:alg-geom/9702016, 1997.

[44] Siddhartha Sahi, Interpolation, integrality, and a generalization of Macdonald's polynomials, Internat. Math. Res. Notices 10 (1996), 457-471. MR 99j:05189b 
[45] T. A. Springer, A construction of representations of Weyl groups, Invent. Math. 44 (1978), no. 3, 279-293. MR 58:11154

[46] Glenn Tesler, Isotypic decompositions of lattice determinants, J. Combin. Theory Ser. A 85 (1999), no. 2, 208-227. MR 2000i:05184

[47] Misha Verbitsky, Holomorphic symplectic geometry and orbifold singularities, Asian J. Math. 4 (2000), no. 3, 553-564, arXiv:math.AG/9903175. CMP 2001:05

[48] Weiqiang Wang, Hilbert schemes, wreath products, and the McKay correspondence, Electronic preprint, arXiv:math.AG/9912104, 1999

Department of Mathematics, University of California at San Diego, La Jolla, CalIFORNIA 92093-0112

E-mail address: mhaiman@math.ucsd.edu 